\title{
Development of direct elemental speciation in solid state materials using pulsed glow discharge mass spectrometry
}

Jennifer N. Robertson-Honecker

West Virginia University

Follow this and additional works at: https://researchrepository.wvu.edu/etd

\section{Recommended Citation}

Robertson-Honecker, Jennifer N., "Development of direct elemental speciation in solid state materials using pulsed glow discharge mass spectrometry" (2008). Graduate Theses, Dissertations, and Problem Reports. 2721.

https://researchrepository.wvu.edu/etd/2721

This Dissertation is protected by copyright and/or related rights. It has been brought to you by the The Research Repository @ WVU with permission from the rights-holder(s). You are free to use this Dissertation in any way that is permitted by the copyright and related rights legislation that applies to your use. For other uses you must obtain permission from the rights-holder(s) directly, unless additional rights are indicated by a Creative Commons license in the record and/ or on the work itself. This Dissertation has been accepted for inclusion in WVU Graduate Theses, Dissertations, and Problem Reports collection by an authorized administrator of The Research Repository @ WVU.

For more information, please contact researchrepository@mail.wvu.edu. 


\title{
Development of Direct Elemental Speciation in Solid State
}

\section{Materials Using Pulsed Glow Discharge Mass Spectrometry}

Jennifer N. Robertson-Honecker

\author{
Dissertation submitted to the \\ Eberly College of Arts and Sciences \\ at West Virginia University \\ in partial fulfillment of the requirements \\ for the degree of
}

Doctor of Philosophy In Chemistry

\author{
Fred L. King, Ph.D., Chair \\ Terry Gullion, Ph.D. \\ Lisa Holland, Ph.D. \\ Earl Scime, PhD. \\ Ronald B. Smart, Ph.D. \\ C. Eugene Bennett Department of Chemistry \\ Morgantown, West Virginia \\ 2008
}

Keywords: Pulsed glow discharge, Mass Spectrometry, Optical Emission Spectroscopy, Perturbation pulse, Langmuir Probe, Speciation

Copyright 2008 Jennifer N. Robertson-Honecker 


\title{
ABSTRACT \\ Development of Direct Elemental Speciation in Solid State Materials Using Pulsed Glow Discharge Mass Spectrometry
}

\author{
Jennifer N. Robertson-Honecker
}

Among the various elemental mass spectrometry techniques, glow discharge mass spectrometry is recognized for its ability to provide direct determination of trace elements present in solid state samples. In the present work, a pulsed glow discharge time of flight mass spectrometry method is developed for the direct speciation of chromium in solid state samples. In initial investigations, time-resolved power perturbation and Langmuir probe studies were undertaken to elucidate the excitation and ionization processes in a pulsed glow discharge plasma. The experimental data provides further insight into the energy transfer processes that occur at different spatial locations and in different temporal regimes of these pulsed glow discharge plasmas. Of greatest interest is the afterpeak regime, in which dissociative recombination between molecular argon ions $\left(\mathrm{Ar}_{2}^{+}\right)$and electrons is thought to be the principal process responsible for emission enhancements. Careful tuning of the operating parameters within this time regime yields the plasma chemistry that favors cluster ion formation. Unique mass spectral features found in the afterpeak permit differentiation between the trivalent and hexavalent forms of chromium, (CrIII) and (CrVI) respectively, in chromium oxide samples. 
To my daughter Helen, whose inquisitive mind and curious nature reminds me every day why I started studying science in the first place.

In loving memory of my Granny Loretta, who never let me take for granted the opportunities available to women today or the struggles that still lie ahead and who showed me that it's never too late to change your life for the better. 


\section{Acknowledgements}

Very few men are wise by their own council, or learned by their own teaching. For he that was only taught by himself, had a fool for a master.--Ben Jonson

To say the path that this once insecure elementary education major took to become the outspoken chemist you see today wasn't a straight one is obvious, to describe it as meandering is still a bit of an understatement. Nevertheless, each twist and turn stands as a true testament to how good teachers can change lives. First l'd like to thank my mentor Dr. Erica Harvey. She and the other chemistry professors at Fairmont State University took such great care in developing chemistry laboratory experiments that were as fun as they were informative, that I promptly switched my major in my sophomore year of college. At Fairmont State my interest in science was nurtured into a true passion and I'm positive that without Dr. Harvey's influence, I wouldn't be here today.

Second, l'd like to thank my advisor Dr. Fred King. His instruction and advice have greatly improved my competence and confidence as both a scientist and educator, and his encouragement ultimately motivated me to obtain a $\mathrm{PhD}$ rather than a Masters degree. I'd also like to thank my fellow research group members over these past five years for their scientific contributions, support, and friendship: Lei Li, Na Zhang, Megan DeJesus, YuChen Lu, Ting Zhao, and Guodong Gu; as well as undergraduate assistants Alex Pavkovich and Vishal Vagehla who contributed significantly to the data collection. I also wish to express my gratitude to Dr. Amy Keesee for her collaborative support on the Langmuir probe investigations. 
I am grateful to my committee members: Dr. Earl Scime, Dr. Lisa Holland, Dr. Ronald Smart, Dr. Kenneth Showalter, and Dr. Terry Gullian. Their support as instructors, collaborative researchers, and mentors has been invaluable. Much of the work presented here would not have been possible without the technical assistance of Don Feathers, Allen Burns, Phillip Tucker and J.R. Taylor. Thanks also to Dr. Michelle Richards-Babb for her mentorship as an educator and in education research.

Finally and most importantly, l'd like to thank my family. Enormous gratitude to my parents for always encouraging me to follow my dreams, to my sister Heather for being the best friend anyone could have, and to my brother Chris for reminding me not to take life too seriously. Foremost, I want to thank my husband Liam, whose love and support have made all of this possible. 


\section{Table of Contents}

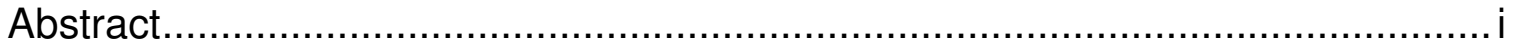

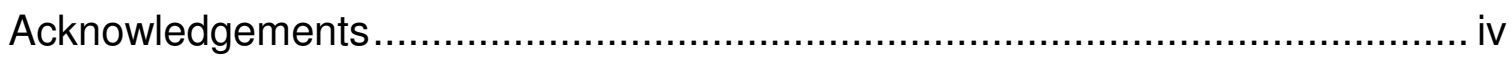

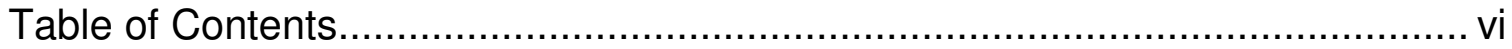

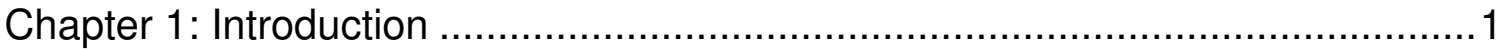

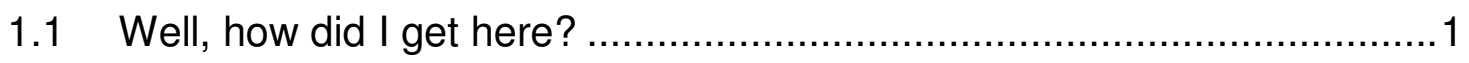

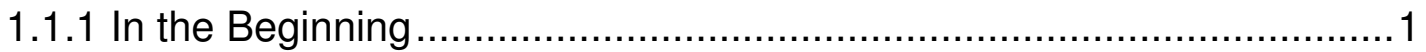

1.1.2 Heralding in the Instrumental Revolution .....................................

1.1.3 Time-of-Flight Mass Spectrometry ................................................ 4

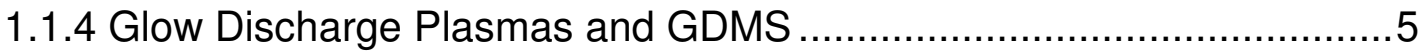

1.2. So what is a glow discharge plasma anyway? ..................................... 7

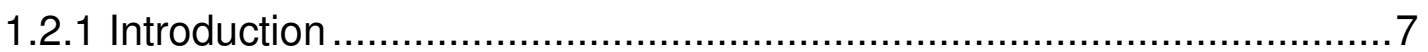

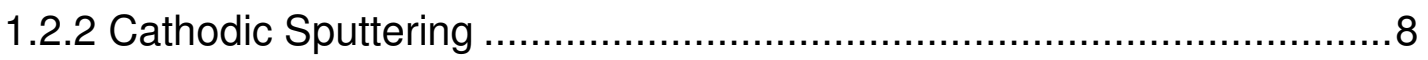

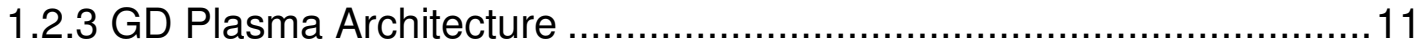

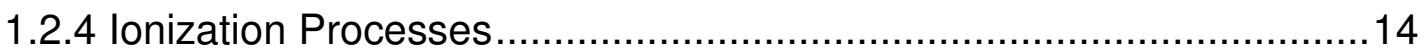

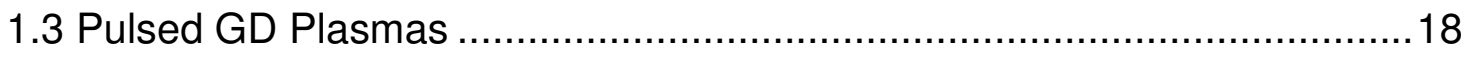

1.3.1 Introduction: Enhancement of Atomization and Ionization .................18

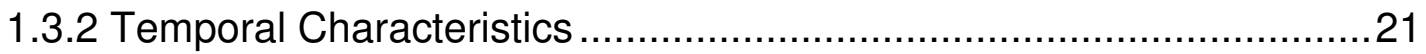

1.3.3 Glow Discharge Plasma Time-of-Flight Mass Spectrometry..............25

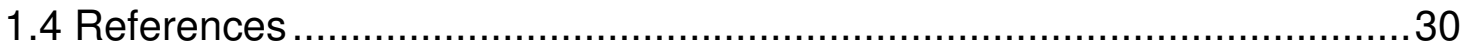

Chapter 2: Electronic Perturbation Investigations into Excitation and Ionization in the Millisecond Pulsed Glow Discharge Plasma ................................................32

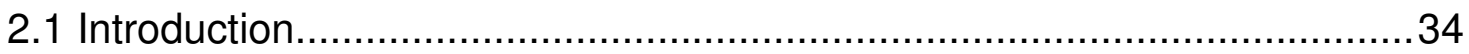

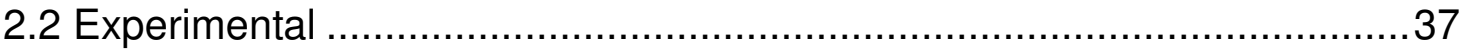

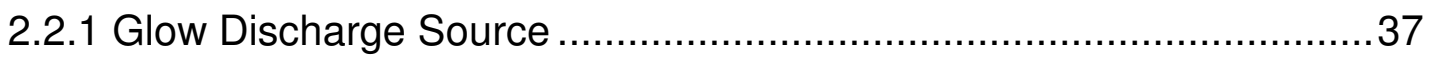

2.2.2 Atomic Emission Measurements..................................................40

2.2.3 Atomic Absorption Measurements ................................................ 40

2.2.4 Power Modulation to Yield a Perturbation Pulse...............................44

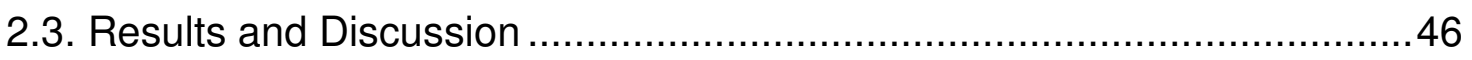

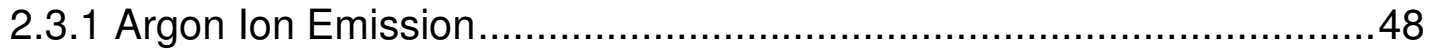




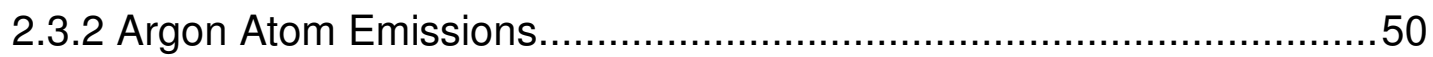

2.3.3 Argon Metastable Atom Absorption .............................................54

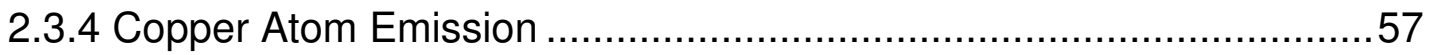

2.3.5 Variation of the Perturbation Pulse in Time.......................................61

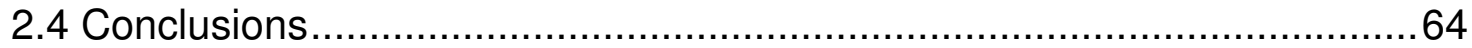

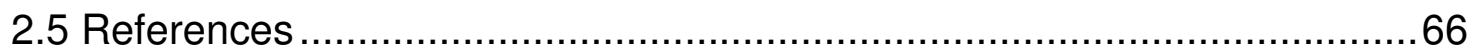

Chapter 3: Spatial, Spectral, and Temporal Characteristics of a Millisecond Pulsed Glow Discharge: Electron Density \& Temperature ................................68

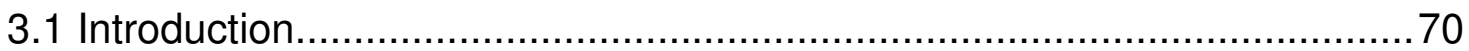

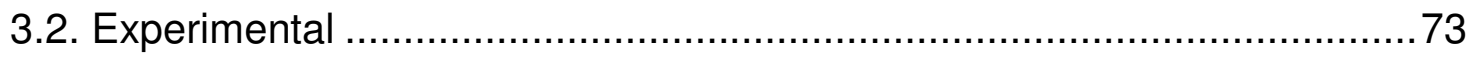

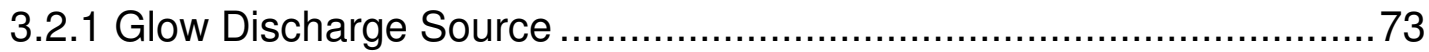

3.2.2 Langmuir Probe Construction ........................................................ 74

3.2.3 Langmuir Probe I-V Characteristic Measurements ............................79

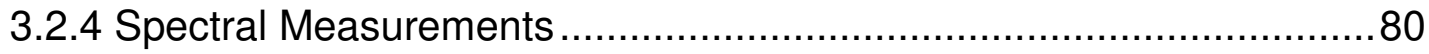

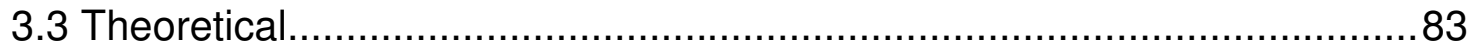

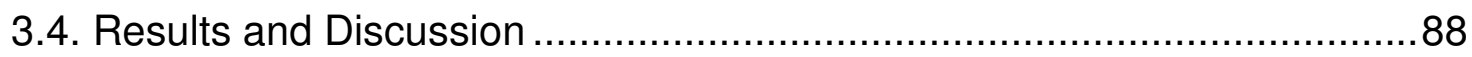

3.4.1 Temporal Langmuir Probe I-V Characteristics ................................88

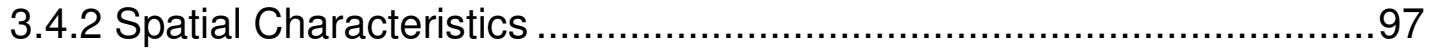

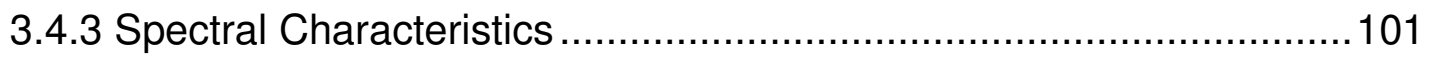

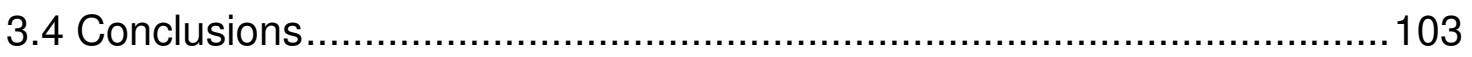

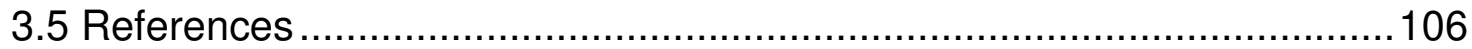

Chapter 4: Direct Chromium Speciation in Solid State Materials - A GDMS

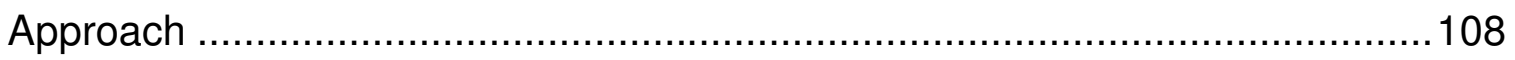

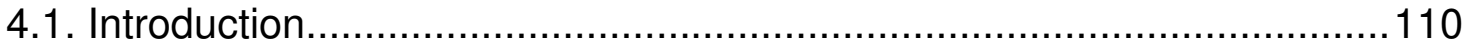

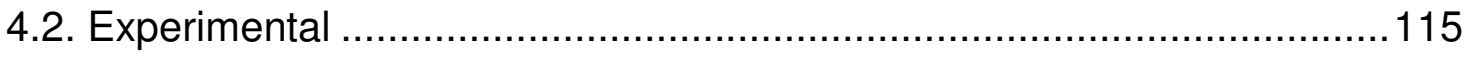

4.2.1 Pulsed Glow Discharge Plasma Device ........................................117

4.2.2 Chromium Sample Cathode Production.........................................117

4.2.3 Time-of-Flight Mass Spectrometer.............................................118

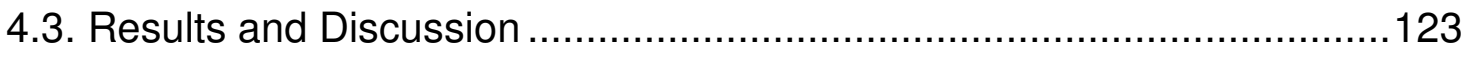

4.3.1 Evaluation of Temporal Regimes .............................................123

4.3.2 Evaluation of Operating Parameters ...........................................129 
4.3.3 Speciation and Quantitative Analysis..........................................134

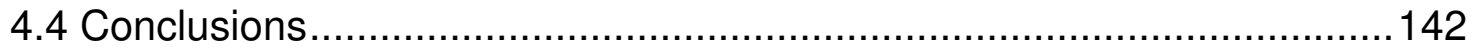

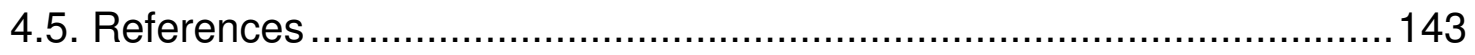




\section{Chapter 1}

Introduction

\subsection{Well, how did I get here?: A Historical Perspective of Glow Discharge Mass Spectrometry}

\subsubsection{In the Beginning}

Although much of early chemistry was analytical in nature, the scientific discipline as we know it today didn't begin until the $19^{\text {th }}$ century. The beginnings of modern analytical chemistry, the branch of chemistry concerned with the separation, identification and determination of the constituents making up a sample of matter and their relative quantities [1], can be traced back to a textbook on quantitative analysis written by Karl Remigius Fresenius. Written in 1846, this text described the known gravimetric and titrimetric methods of the time. Citing the growing amounts of research on chemical analysis being done in his and other laboratories, in 1862 Fresenius founded the first journal exclusively devoted to analytical chemistry entitled Zeitschrift für Analytische Chemie [2]. By the latter part of the century the discipline was well established and in 1894, Wilhelm Ostwald described it this way:

"Analytical chemistry, or the art of recognizing different substances and determining their constituents, takes a prominent position among the applications of science, since the questions which it enables us to answer arise wherever chemical processes are employed for scientific or technical purposes. Its supreme importance has caused it to be assiduously 
cultivated from a very early period in the history of chemistry, and its records comprise a large part of the quantitative work which is spread over the whole domain of science" [1].

In the past 100 years since, analytical chemistry has grown from an art to a science. It is hard to imagine a part of modern life that hasn't been touched by the discipline. Pharmaceuticals, foodstuffs, industrial goods and consumer products all utilize methods of chemical analysis to improve quality and insure public safety. Government policies on environmental issues have been shaped by the chemical analysis of our nation's ambient air and water. The Clean Air and Water Acts of 1970 and1977, as well as the eventual ban of tetraethyl lead as an additive in gasoline in 1995 are but a few examples. Today, classical analytical chemistry has expanded to include applications in forensic, environmental, industrial, medical, and biological analytical chemistries. As Ostwald poignantly quipped, analytical chemistry truly is the "handmaiden of the sciences" [2].

\subsubsection{Heralding in the Instrumental Revolution and the Advent of Mass Spectrometry}

The major advances in analytical chemistry are due in no small part to key developments in instrumental analysis. The first instrumental analysis was performed in 1859 by Robert Bunsen and Gustav Kirchoff using their flame emissive spectroscope. The instrument consisted of a carbon disulfide filled prism and a Bunsen burner flame into which salts were introduced on a platinum wire. Further developments in instrumentation ensued and in 1905, the predecessor to the first mass spectrograph was produced by J. J. Thomson. 
Inspired by the observations of "positive rays" (ion beams) made by Eugene Goldstein and Wilhelm Wein, Thomson channeled a stream of ionized neon through both a magnetic and an electric field. His device used ions with a wide range of velocities and a photographic plate onto which their deflection through the fields could be measured. These experiments provided the first observational evidence of the existence of ions, ${ }^{20} \mathrm{Ne}$ and ${ }^{22} \mathrm{Ne}$ [2].

The first working version of the modern mass spectrometer was designed by Thomson's student, Francis W. Aston, in 1919. His instrument used a discharge bulb as the ion source and a method of electromagnetic focusing that could focus both the velocity and the direction of the ions, enabling very slight differences in mass to be separated and measured. When deciding on a name for his instrument, Aston commented

"Since it is a close analogue of the ordinary spectrograph, and gives a spectrum depending upon mass alone, the instrument is called a 'massspectrograph' and the spectrum it produces a 'mass spectrum'” [2]. Aston's mass spectrograph had a mass resolving power of 130 allowing him to identify 212 of the naturally occurring isotopes [3].

By the 1930's, major advances in high-vacuum techniques and the commercial availability of electrometer vacuum tubes improved the accuracy and ease with which mass spectrometry measurements could be made. These innovations along with the advent of World War II and the development of the atomic bomb spawned a heightened interest in mass spectrometry. In the early 1940's the University of Minnesota was awarded contracts by the Office of 
Scientific Research and Development to construct a pilot number of mass spectrometers for the isotopic analysis of uranium and hydrogen as well as helium sensitive leak detectors. The Minnesota design for the sector helium leak detector was commercialized by the General Electric Company in 1942 [2, 4].

\subsubsection{Time-of-Flight Mass Spectrometry}

Prior to the late 1940's, the separation of ions in mass spectrometry was preformed via their deflection in electric and magnetic fields. These instruments required large, heavy electromagnets and very precise power supplies. In 1946, W. E. Stephens proposed a method for the separation of ions based on the time it would take them to fly down a long tube. Stephens postulated that if a pulse of ions were given the same start time with the same amount of energy, then, according to the fundamental relationship between kinetic energy $(K E)$, mass $(m)$, and velocity $(v)$ (shown in Equation 1.1), ions of different masses would have different velocities and thus different flight times down the tube.

$$
K E=1 / 2 m v^{2}
$$

(Equation 1.1)

Using this concept, the "Velocitron" was built two years later by A. E. Cameron and D. F. Eggers. Subsequent modifications made by Wiley and McLaren in the early 1950 's became the prototype of the modern time-of-flight mass spectrometer (ToFMS) and was commercially marketed by the Bendix Corporation. This instrument had a mass range from 1-600 and could collect over 10,000 spectra per second [4]. 


\subsubsection{Glow Discharge Plasmas and GDMS}

As stated earlier, the predecessor to the first mass spectrometer was produced in 1905 by J. J. Thomson when he was investigating the "positive rays" from what was effectively a glow discharge source. However, work with glow discharges had actually begun over a century earlier. In 1785, William Morgan communicated in a paper to the Royal Society of London on phenomena produced by the passage of electric discharges in a glass tube. Morgan found that when the vacuum was as perfect as he could make it, no electric discharge could pass, but upon admitting a minuscule amount of air, the glass glowed with a "beautiful green colour", which changed from green to blue and so on to violet and purple as more gas was allowed to enter the tube [5].

In the late 1850's, Julius Plückner showed that by applying an electric field to two electrodes within a vacuum tube, he could cause the gas to glow with a greenish light near the positive electrode, which he named the cathode [6]. In addition, Plückner was able to demonstrate that a magnet affected the direction of the discharge rays. Eugene Goldstein, also pursuing research in this area, named these rays "cathode rays". Goldstein continued his research with discharge tubes and in 1886, using a perforated cathode, observed faint streamers emerging from behind the holes in the cathode. He coined these rays "kanalstrahlen" or canal rays. In 1895, J. Perrin demonstrated the associated negative charge of cathode rays when he was able to bend them using a magnetic field onto a faraday cup connected to an electrometer. A few years later, W. Wein showed that, while a fairly weak magnetic field could easily deflect cathode rays, it took a very strong magnetic field to affect the path of canal rays 
to any modest degree. Furthermore, he concluded that since the deflection of canal rays were opposite to that of cathode rays, they were positive. It was these early observations of glow discharge plasmas in vacuum tubes that led to our present views of atoms, ions, protons, and electrons [4].

In 1954, N. B. Hannay and A. J. Ahearn introduced spark source mass spectrometry (SSMS) a technique that utilized a high voltage electric discharge, or spark, to atomize and ionize solid samples within a vacuum [Hoffmann, 2004]. SSMS was only the second instrumental analysis tool capable of analyzing solids, the first being the flame emissive spectroscope invented by Bunsen and Kirchoff nearly 100 years earlier [4]. Unfortunately, spark sources were very unstable making them unsuitable for quantitative analysis; their ultimate successor became glow discharge mass spectrometry (GDMS).

In 1971, motivated by the needs of material science industries who were utilizing glow discharge (GD) plasmas for thin film deposition, etching, and surface treatment, W. Coburn and co-workers decided to investigate glow discharge plasmas by mass spectrometric methods [7]. Coburn et al. quickly realized the ability of glow discharge to both atomize and ionize solid cathode materials [8]. These initial studies were followed by the work of W. Harrison's group who were working on a glow discharge alternative to SSMS, the results of which further confirmed glow discharge mass spectrometry (GDMS) as a method for elemental analysis for solid samples [9]. The use of GD plasmas is now well established for the direct determination of bulk and trace elements in solid state samples. Biological compounds, plastics, soils, and coal fly ash are but a few 
examples of materials studied by GD [10,11, 12, 13]. Furthermore, when modified by time gating and combined with separation techniques like gas chromatography, chemical speciation of volatile organic and organometallic compounds are possible $[14,15,16]$.

\subsection{So what is a glow discharge plasma anyway?}

\subsubsection{Introduction}

A plasma is a partially ionized gas consisting of equal numbers of positive and negative charges, mostly electrons and positive ions. The degree of ionization is on the order of $10^{-4}$ (fractional proportion), so the gas consists mostly of neutral gas atoms or molecules but can also contain free radicals, electrons, and photons. A glow discharge (GD) is a type of plasma in which only the electrons are hot $(\sim 25,000-50,000 \mathrm{~K})$ and the neutrals and ions are at modest temperatures $(\sim 500 \mathrm{~K})$ due to their large masses. This is in marked contrast to the kinetic temperatures of neutrals and ions in combustion flames $(\sim 2,500 \mathrm{~K})$ or inductively coupled plasmas ( 10,000 K) [17]. GD plasmas are created in a reduced-pressure environment (.02-1 Torr) when the voltage applied between two electrodes exceeds the energy necessary to cause the breakdown of the discharge gas, usually argon. This breakdown leads to the creation of electronion pairs.

When a potential difference is applied between the two electrodes in the GD chamber, free electrons in the chamber are accelerated away from the cathode, occasionally colliding with argon atoms in the discharge gas. This gives 
rise to ionization and excitation processes, creating argon ions and new electrons. The newly formed argon ions, $\mathrm{Ar}^{+}$, are then accelerated toward the cathode. When they strike the cathode surface, they can release "secondary electrons" which go on to create new argon ions and electrons thereby generating a self-sustaining plasma. GD owes its name to the fact that the plasma is luminous. This is caused by electron excitation processes between electrons and discharge gas atoms in the GD chamber. Following impact, the atom absorbs energy, exciting electrons which later relax and emit photons giving the plasma its characteristic glow [18]. An argon plasma emits a blue glow.

\subsubsection{Cathodic Sputtering}

The analytical utility of glow discharge mass spectrometry (GDMS) lies squarely with a process called sputtering. Sputtering is a process of momentum transfer that occurs when a surface is bombarded by fast heavy particles causing atoms of the surface to be ejected in all directions. In GD, the two electrodes typically consist of a cathode composed of the sample material to be analyzed and the chamber walls serving as the anode. When highly energetic argon ions bombard the cathode surface, their kinetic energy is transferred into the cathode lattice. This impact can result in one or all of the following phenomena (see Figure 1.1) [19]:

a) Reflected lon: the ion is reflected and most likely neutralized in the process 
b) Impacted lon and Structural Changes: the ion becomes implanted within the cathode and can create an altered volume of lattice defects

c) Secondary Electron Ejection: an electron is ejected from the cathode surface

d) Cathode Atom Ejection: the impact creates a series of collisions among atoms in the cathode material ultimately ejecting one of these atoms

The series of collisions between cathode atoms is known as a "collision cascade" and the atom ejection process is called "sputtering". Two things must happen before an atom is sputter ejected from the surface of the cathode: 1) the collision cascade must occur along the surface of the cathode rather than into its interior, and 2) the collision between the final atoms must be enough to exceed the lattice binding energy of the cathode material [19]. It is for this reason that the sputtered atom population accounts for less than $1 \%$ of the total plasma species [7]. 


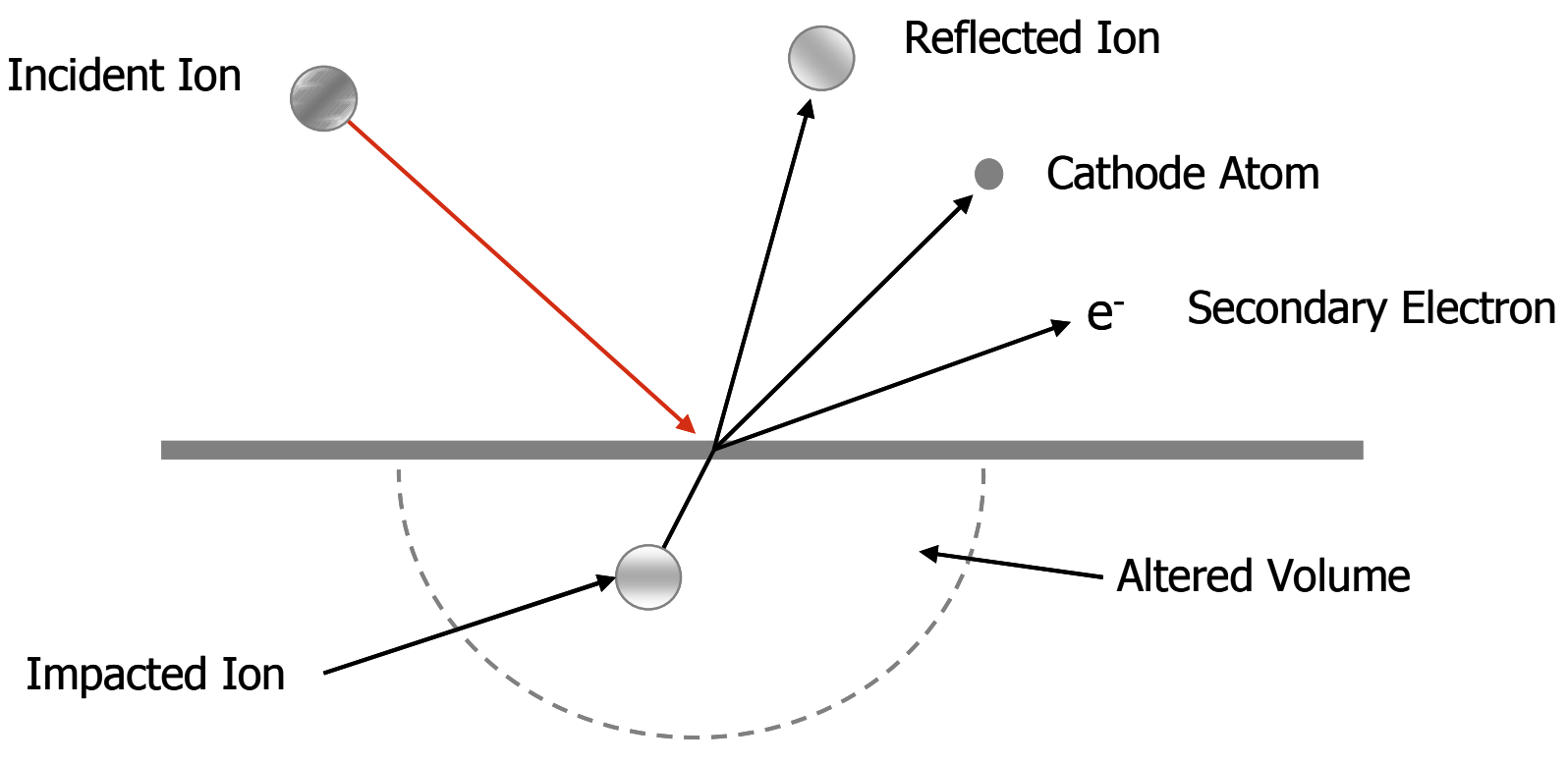

Figure 1.1. Schematic representation of the possible glow discharge ion interactions at the surface of the cathode [Adapted from 19]. 


\subsubsection{GD Plasma Architecture}

The architecture of a glow discharge (GD) consists of several regions, noted mostly for their luminosity. These regions differ greatly in the types of ionization mechanisms and the populations of charged species found therein. For the GD setup used in these studies, only three regions are available for analysis; the cathode dark space, the negative glow, and the Faraday dark space.

Adjacent to the cathode is a noticeably dark region known as the cathode dark space (CDS). Although the CDS is very narrow, the majority of the potential difference between the two electrodes, in our case the cathode and the grounded chamber wall, drops across this region making it the area of strongest electric field. In this region, ions are accelerated into the cathode material with enough force to cause the emission of a cathode atom or a secondary electron from the cathode, called sputtering. Electrons, on the other hand, are repulsed in this area creating a sheath of positive space charge in front of the cathode, the thickness of which is approximately three times the Debye length $\left(\lambda_{D}\right)[19,20]$.

The bright luminous portion of the plasma bordering the CDS is the negative glow region (NG). Here, two general types of electrons can be found: energetic (fast) secondary electrons which have yet to lose their energy by collision, and thermalized (slow) electrons that have been collisionally cooled or created via ionization reactions [20]. The fast electrons are responsible for electron ionization and are essential to a sustained GD, whereas the thermalized electrons give rise to excitation and recombination processes responsible for both the characteristic visible emission of the NG and the "softer" Penning ionization found therein. Due to approximately equal densities of electrons and 
positive ions, $10^{9}-10^{11} \mathrm{~cm}^{-3}$, the NG is essentially field free and its collision and ionization rich environment makes it the region of highest analytical utility [20].

The third and final region in the GD is the Faraday dark space (FDS). This region is marked by low luminosity due to the diminished energies of the postcollisional electrons diffusing from the anode edge of the NG. Here, the electrons begin to "feel" the presence of the positive anode and are accelerated toward it [20]. However, this diffusion is limited by the rate at which the positive ions can also diffuse to the walls, a phenomenon known as ambipolar diffusion [19]. 

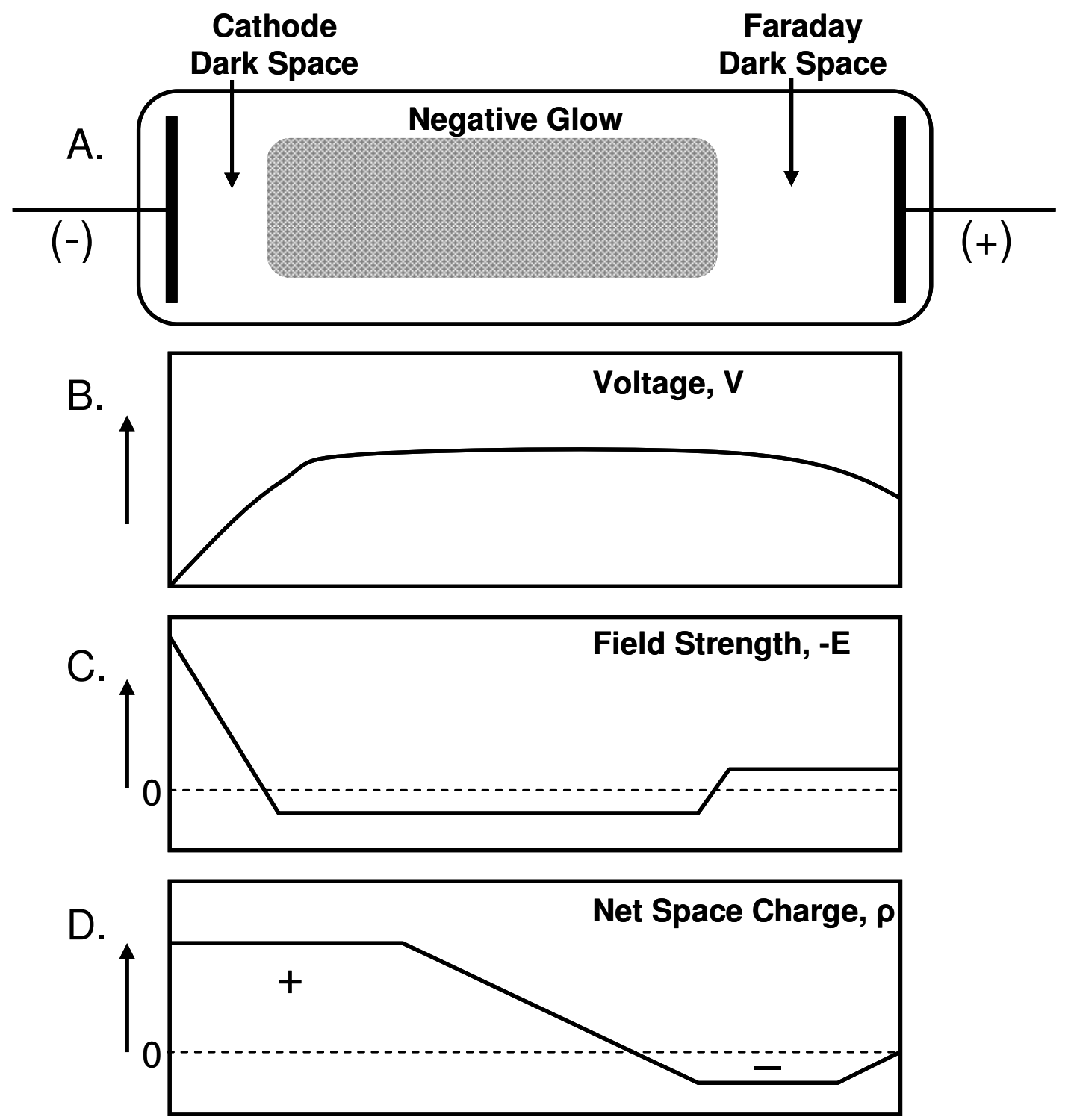

Figure 1.2. Schematic of the A) normal glow discharge architecture showing the B) voltage, C) field strength, and D) net space charge for each area. Although two electrodes are marked for simplicity, the typical GD instrument uses the grounded chamber walls as the anode [Adapted from 20]. 


\subsubsection{Ionization Processes}

Glow discharge (GD) plasmas are rich in ionization mechanisms. The atomization of an analyte in a GD ion source is by virtue of cathodic sputtering, in which ionized discharge gas atoms, created by the breakdown of the discharge support gas, are accelerated toward the cathode where they bombard the surface and "knock off" sample atoms. Once atomized, the sputtered analyte atoms can become excited or ionized in the surrounding plasma via inelastic collisions with various energetic species (i.e. fast electrons, excited and metastable atoms, and ions). Each ionization mechanism imparts a specific amount of energy into the analyte atom or molecule, leading to possible fragmentation and dissociation in addition to ionization and excitation. The three main ionization mechanisms occurring within the GD plasma are electron ionization, charge transfer, and Penning ionization (see Figure 1.3).

\section{Electron Ionization}

Electron ionization is central to a sustained GD plasma due to the fact that the electrons formed through this process can give rise to further ionization. In this ionization mechanism, a primary electron collides with an atom in an inelastic collision to remove an electron from the atom, producing a positive ion and two electrons (Equation 1.2).

$$
e^{-}(\text {fast })+A r \rightarrow 2 e^{-}(\text {slow })+A r^{+}
$$


Because electrons are typically accelerated to energies between 50 and $150 \mathrm{eV}$, electron ionization is considered a "hard" ionization process resulting in high ionization efficiencies and the extensive fragmentation of analyte molecules.

\section{Charge Transfer}

When an argon ion collides with an atom, an electron can be transferred from the atom to the ion. This can occur between two argon atoms, known as symmetric charge transfer (Equation 1.3), or between an argon ion and an analyte atom, asymmetric charge transfer (Equation 1.4).

$$
\begin{aligned}
& \mathrm{Ar}^{+} \text {(fast) }+\mathrm{Ar} \text { (slow) } \rightarrow \mathrm{Ar} \text { (fast) }+\mathrm{Ar}^{+} \text {(slow) } \\
& \mathrm{Ar}^{+}+\mathrm{M} \rightarrow \mathrm{Ar}+\mathrm{M}^{+}+\Delta \mathrm{E}
\end{aligned}
$$

(Equation 1.3)

(Equation 1.4)

Symmetric charge transfer vastly increases the population of fast argon atoms in the negative glow of the plasma; these atoms play an important role in cathodic sputtering. Asymmetric charge transfer can only occur if the ionization potentials of the argon ion $(15.76 \mathrm{eV})$ and the resulting analyte ion are similar $( \pm 0.2 \mathrm{eV})$, making it a highly selective ionization process [18].

\section{Penning lonization}

Besides the favorable characteristics of chemical inertness and large atomic weights necessary for sputtering in GD, noble gases have high-lying longlived metastable states. Atoms in a metastable electronic state (triplet), known as metastable atoms, have long radiative lifetimes because their relaxation to lowerenergy levels is spin-forbidden [20]. Metastable argon atoms are created via 
recombination processes involving argon ions and thermalized (slow) electrons (Equation 1.5). If a metastable argon atom collides with an analyte atom, the potential energy of the metastable argon (11.55 and $11.62 \mathrm{eV}$ ) can be used for ionization, but only if the ionization potential of the analyte atom lies below the metastable energies. This process is known as Penning ionization (Equation 1.6).

$$
\begin{aligned}
& \mathrm{e}^{-}(\text {slow })+\mathrm{Ar}^{+} \rightarrow \mathrm{Ar}^{\mathrm{m}} \\
& \mathrm{Ar}^{\mathrm{m}}+\mathrm{M} \rightarrow \mathrm{Ar}+\mathrm{M}^{+}+\mathrm{e}^{-}
\end{aligned}
$$

Fortunately, there are very few elements that can't be ionized by this mechanism [18]. For those that can Penning ionize, the process accounts for $40-80 \%$ of their total ionization in a continuous glow discharge [7]. 


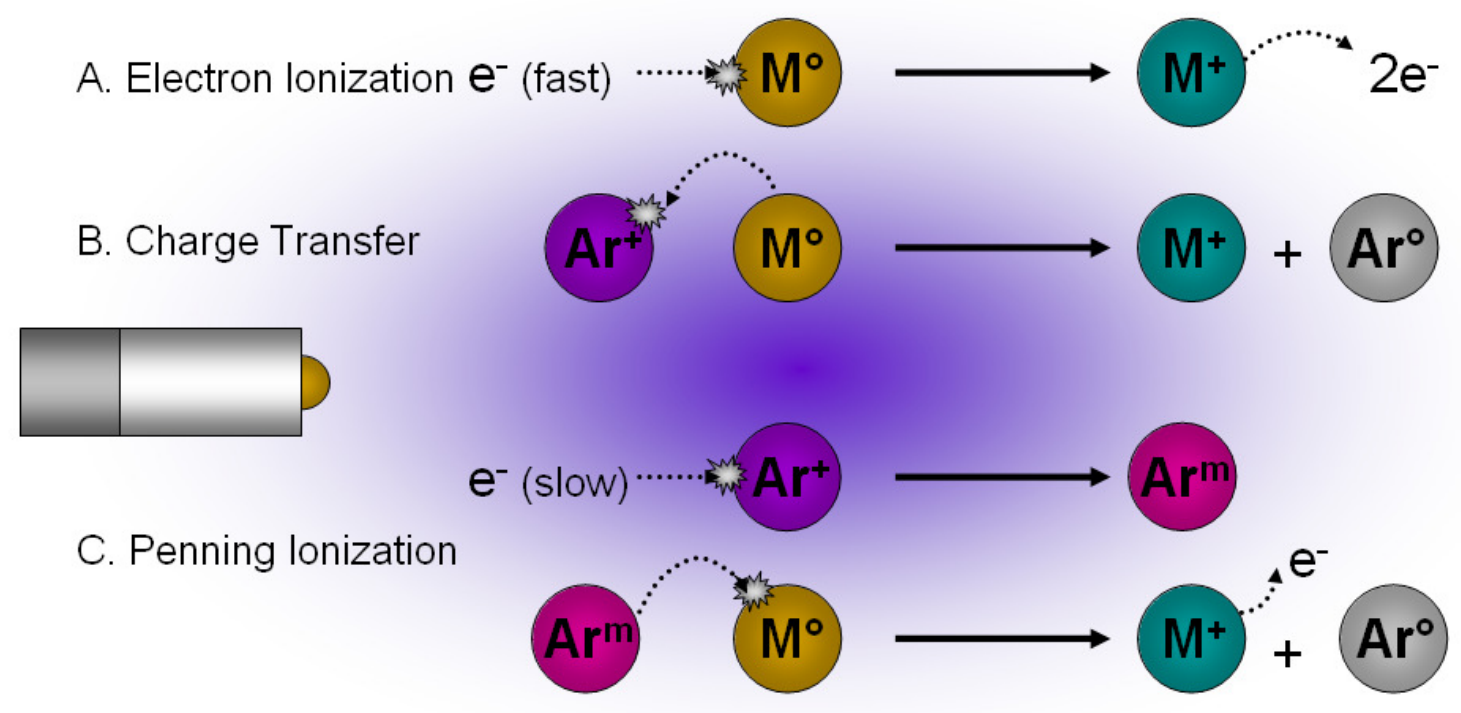

Figure 1.3. lonization processes occurring within the glow discharge plasma include A) electron lonization, B) charge transfer, and C) Penning Ionization. 


\subsection{Pulsed GD Plasmas}

\subsubsection{Introduction: Enhancement of Atomization and Ionization}

In a continuous power glow discharge, the plasma is steady-state with the various ionization mechanisms occurring simultaneously. Pulsed glow discharges, first introduced by Harrison et al, use modulated power cycles generated by a succession of square wave pulses [21]. In such pulsed operation, a relatively brief "power-on" period is followed by a much longer "power-off" period. These pulses can last from microseconds to milliseconds with frequencies up to $200 \mathrm{~Hz}$ [15]. The investigations discussed in Chapters 2 through 5 of this dissertation utilized 5 ms power pulses with a power-off period of $15 \mathrm{~ms}$, giving a $50 \mathrm{~Hz}$ pulse rate with a $25 \%$ duty cycle.

Several analytical advantages arise from pulsing the GD plasma. First, the pulsed power mode provides the sample material (cathode) with a "cooling off" period during each power-off phase. This affords the application of higher instantaneous power, yielding increases in atomization, excitation, and ionization, without provoking thermal degradation of the sample. It has been reported that a pulsed glow discharge with the same average power input as a continuous glow discharge yields a 100-fold increase in signal intensity [22]. Second, when operated in pulsed mode, spatial and temporal separation of the different ionization mechanisms becomes possible through the creation of three distinct time regimes during each on/off cycle: a prepeak dominated by electron impact ionization, an afterpeak dominated by Penning ionization, and a steady state plateau which arises from a mixture of electron impact, charge transfer, and 
Penning ionizations (see Figure 1.3). Using time-gated detection, these transient ionization periods permit temporal discrimination between analyte signal intensities and those of the discharge gas and background contaminants, dramatically enhancing the signal to noise ratio. 


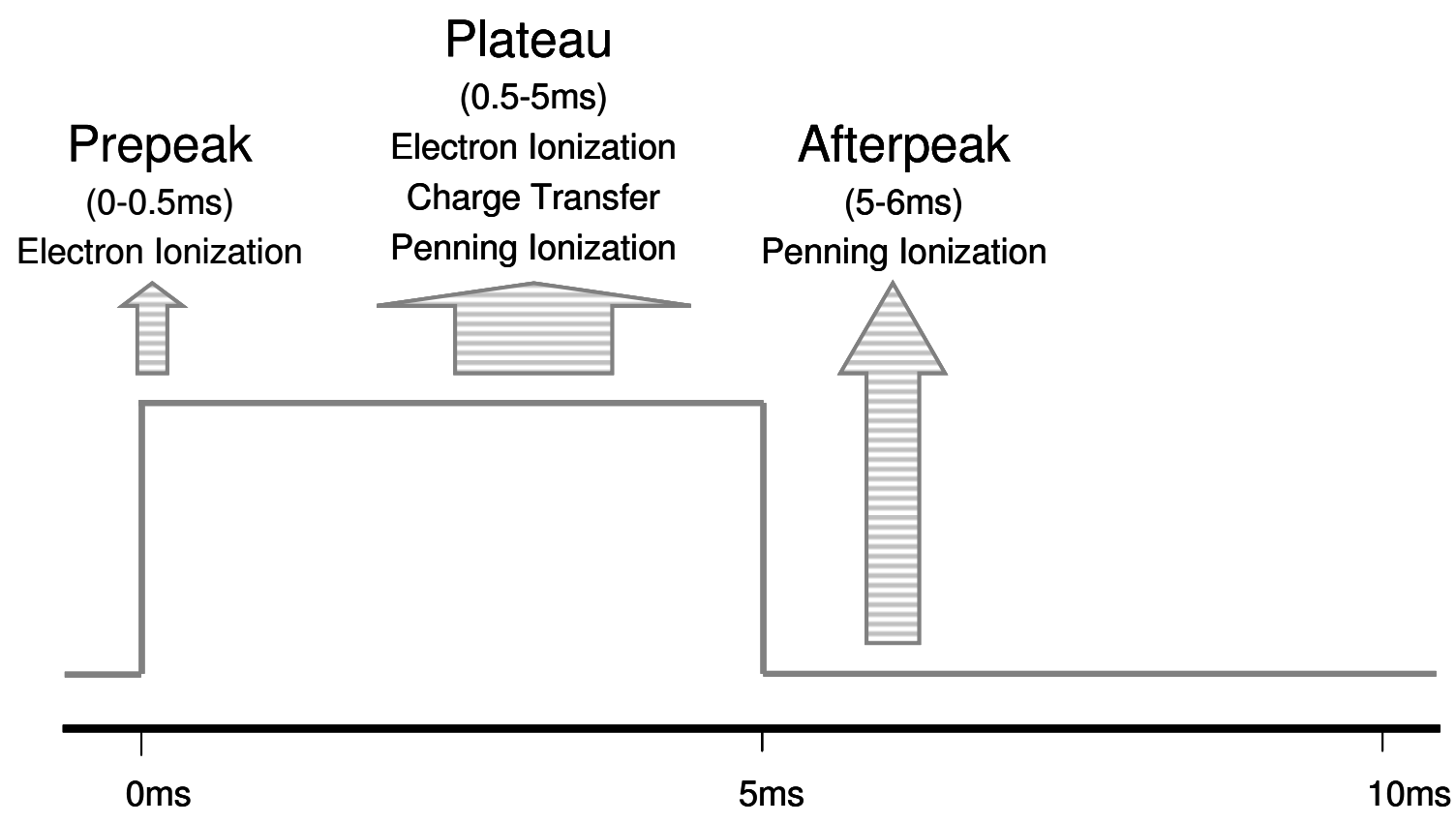

Figure 1.4. Schematic representation of a millisecond GD power pulse and the dominate ionization mechanisms associated with each temporal regime. Electron ionization dominates in the prepeak to yield elemental information; an equilibrium in the plateau between charge transfer, electron ionization, and Penning ionization yields structural and fragmentation information; and in the afterpeak Penning ionization dominates to yield molecular ion information. 


\subsubsection{Temporal Characteristics}

Because of their light mass and high mobility, electrons are the first species in the plasma to react to the power onset. Upon power initiation, a highly energetic electron population forms and electron ionization becomes dominant. During this period, 0.1 to $0.5 \mathrm{~ms}$ into the GD pulse, the discharge gas is broken down and ionized resulting in a surge of ion intensities. Electron ionization is a very energetic and therefore "hard" ionization mechanism. Time-gated analysis of this time regime, denoted the prepeak, gives rise to extensive fragmentation and elemental information, but is mostly overshadowed by strong discharge gas ion intensities, in our case argon $\left(\mathrm{Ar}^{+} 40 \mathrm{~m} / \mathrm{z}\right.$ and $\left.\mathrm{ArH}^{+} 41 \mathrm{~m} / \mathrm{z}\right)$.

As more argon ions form, sputtering is enhanced and signal contributions from analyte species intensify. About $\sim 1 \mathrm{~ms}$ into the GD pulse, the large electron population diminishes and dominant ionization mechanisms equilibrate into a mixture of electron impact, charge transfer, and Penning ionizations. At this time, the ions of both gas phase and sputtered species can be observed, the intensities of which remain at a steady-state value until power termination (1 to 5 $\mathrm{ms}$ ). The plasma configuration during this time regime, referred to as the plateau, closely resembles that of a continuous GD plasma. Time-gated analysis of the plateau regime yields structural and fragmentation information about the analyte.

Upon power termination, both electron ionization and charge transfer processes halt and the ions formed by these processes quickly decay to a baseline value. At this time, Penning ionization dominates as argon ions and thermalized electrons undergo recombination processes to form metastable argon ions. Metastable atoms efficiently Penning ionize the plasma species of 
lower ionization potentials, generally sputtered analyte atoms. During this time regime, known as the afterpeak (5.1 to $6 \mathrm{~ms}$ ), analyte ion signal profiles maximize. Because Penning ionization is limited by the potential energies of metastable argon atoms, $11.55 \mathrm{eV}$ and $11.72 \mathrm{eV}$, the afterpeak regime can provide for energy-tuned, selective ionization. For elemental analysis, this permits temporal discrimination between signals of analytes and their interferences. The "soft" Penning ionization of the afterpeak produces principally molecular ions to provide molecular weight information for analytes.

Figures 1.5 and 1.6 show the temporal ion profiles of a copper cathode, used as the analyte material with argon as the discharge gas for optical emission and mass spectral data respectively. 


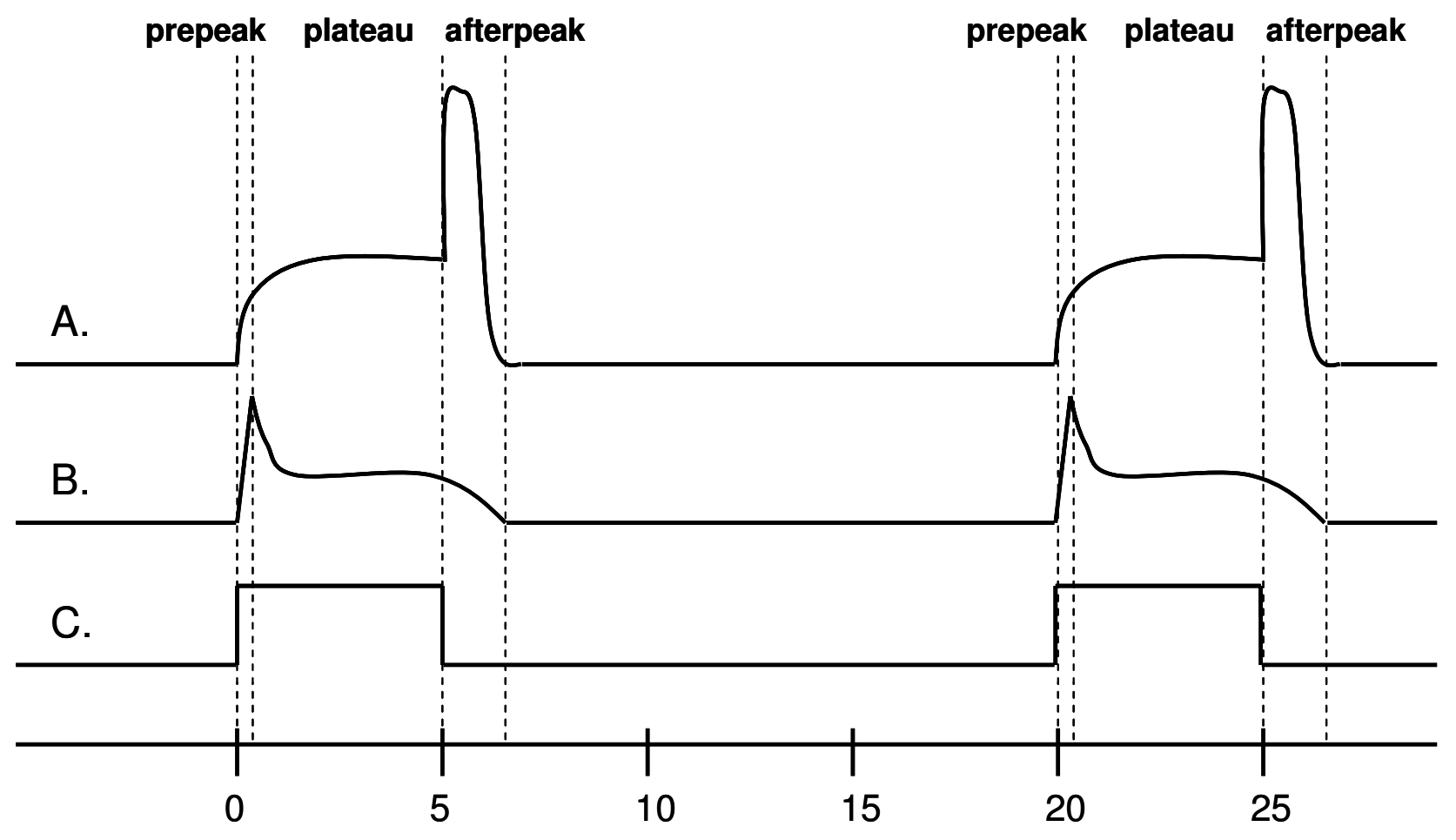

Figure 1.5. Schematic optical emission profiles of A) sputtered copper analyte material, B) argon discharge gas, and C) the applied millisecond power pulse $(5 \mathrm{~ms}$ at $50 \mathrm{~Hz})$. 


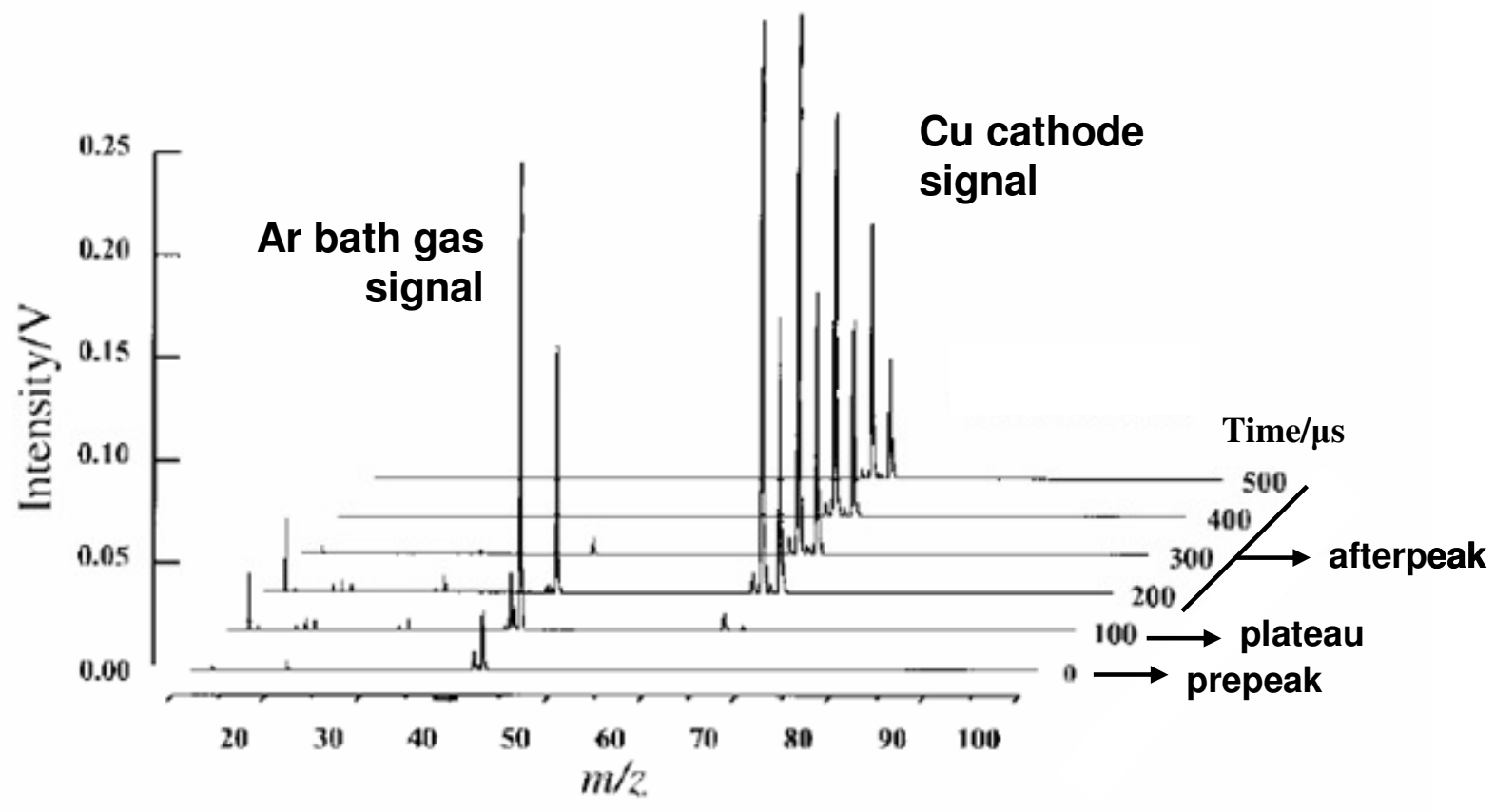

Figure 1.6. Time resolved microsecond pulsed $(20 \mu \mathrm{s}$ at $100 \mathrm{~Hz}) \mathrm{GD}$ ToFMS of a copper cathode in argon discharge gas. At $100 \mu$ s after the pulse initiation the argon discharge gas signal intensities $\left(\mathrm{Ar}^{+} 40\right.$ and $\mathrm{ArH}^{+}$ 41) dominate. Contaminant ions due to molecular oxygen and water and their associated fragments can also be seen. At $200 \mu \mathrm{s}$ and beyond, the copper analyte signal $\left(\mathrm{Cu}^{+}\right.$isotopes, 63 and 65$)$ increase to a maximum while the discharge gas and contaminant signals become insignificant [14]. 


\subsubsection{Glow Discharge Plasma Time-of-Flight Mass Spectrometry}

In time-of-flight mass spectrometry (ToFMS), ions enter the mass spectrometer and fill an extraction region defined by a repeller plate $(R)$ and an extraction grid $\left(G_{0}\right)$ where they are held until the desired injection time. At a set delay time, the repeller is pulsed to a high voltage, producing a linear potential field between $R$ and $G_{0}$ that injects the ions into the acceleration region between $\mathrm{G}_{0}$ and $\mathrm{G}_{1}$ (see Figure 1.6). The acceleration region exerts a constantacceleration onto the ions before they enter the field-free drift region (flight tube). Ions separate in the flight tube based on differences in velocity, with light ions achieving higher velocities than heavier ones. A detector positioned at the end of the flight tube records the arrival time of the ions to produce a complete mass spectrum $[23,24]$.

The fundamental theory behind the creation of a mass spectrum in a ToFMS is dependent on the notion that the potential energy of a charged particle in an electric field is related to the strength of the electric field $(\mathrm{eV})$ and to the charge of the particle $(z)$.

$$
P E=z e V
$$

(Equation 1.7)

When the charged ion is accelerated between regions $G_{0}$ and $G_{1}$ (Figure 1.6), potential energy is converted into kinetic energy and Equations 1.1 and 1.7 become equivalent.

$$
z e V=1 / 2 m v^{2}
$$

The time-of-flight tube, region $\mathrm{G}_{1}$ to the detector, is field free allowing for constant velocity. The flight path (d) along this region is also constant and, since the time 
(t) of the ion's flight is measured by the instrument, the velocity can be calculated.

$$
v=d / t
$$

(Equation 1.9)

Equation 1.9 can now be substituted into Equation 1.8.

$$
z e V=1 / 2 m(d / t)^{2}
$$

(Equation 2.0)

Rearranging to solve for time we find...

$$
t=\frac{d}{\sqrt{2 e V}} \sqrt{\frac{m}{z}}
$$

Where distance (d) and electric field (eV) are constant and known, demonstrating that the flight time of an ion varies with the square root of its mass-to-charge ratio $(\mathrm{m} / \mathrm{z})[25]$

If an ion's flight time was strictly dependent upon its mass-to-charge ratio, the ToFMS would have unlimited resolution. In practice, however, mass resolution is affected by factors that create a distribution in flight times for ions of the same $\mathrm{m} / \mathrm{z}$. These factors include space charge effects, inhomogeneous electric fields, the temporal and spatial spread of the ionization source, and the initial distribution of ion velocities. As a consequence, resolution decreases by increasing the measured time width of the ion packet that reaches the detector [25]. Resolution in time-of-flight can be calculated in two ways; based on Equation 2.2 or from the flight times.

$$
\boldsymbol{R}=\frac{\boldsymbol{m}}{\Delta \boldsymbol{m}}
$$

From the flight time equation (2.1) we see that the mass resolving power is equal to 


$$
R=\frac{t}{2 \Delta t}
$$

(Equation 2.3)

Setting Equations 2.2 and 2.3 equal and rearranging for the change in mass we see

$$
\Delta m=\frac{m}{t} 2 \Delta t
$$

revealing that the mass width is proportional to $\Delta t$ and the mass of the ion [26].

To ensure that all ions receive the same start time down the flight tube, ion sources for use with ToFMS must either be pulsed or gated and can be oriented orthogonally, at a right angle to, or axially, in line with the acceleration of the ions. In the investigations discussed herein, the GD ion source is pulsed using either a direct current (DC) or radio frequency (rf) generated power source. For DC, the power pulses are generated using a function generator and an electronic high voltage pulser. The function generator is programmed to produce the desired waveforms, which in turn triggers the pulser to gate the high voltage output from the power supply resulting in precisely controlled square wave power pulses. In rf, the internal pulsing mode of the power supply system permits control of applied power, pulse width, and duty cycle.

In summary, through the use of a pulsed glow discharge plasma source, analyte molecules are exposed to a series of transient ionization periods within each pulse cycle. The ionization mechanisms within these distinct temporal regions range from "hard" to "soft", allowing for a highly tunable ion source. When combined with a time-gated detection method such as time-of-flight mass spectrometry, the investigator is afforded the selection of a particular time 
regime/plasma chemistry. In this way, one can obtain elemental and/or molecular information in determining a sample's composition. 

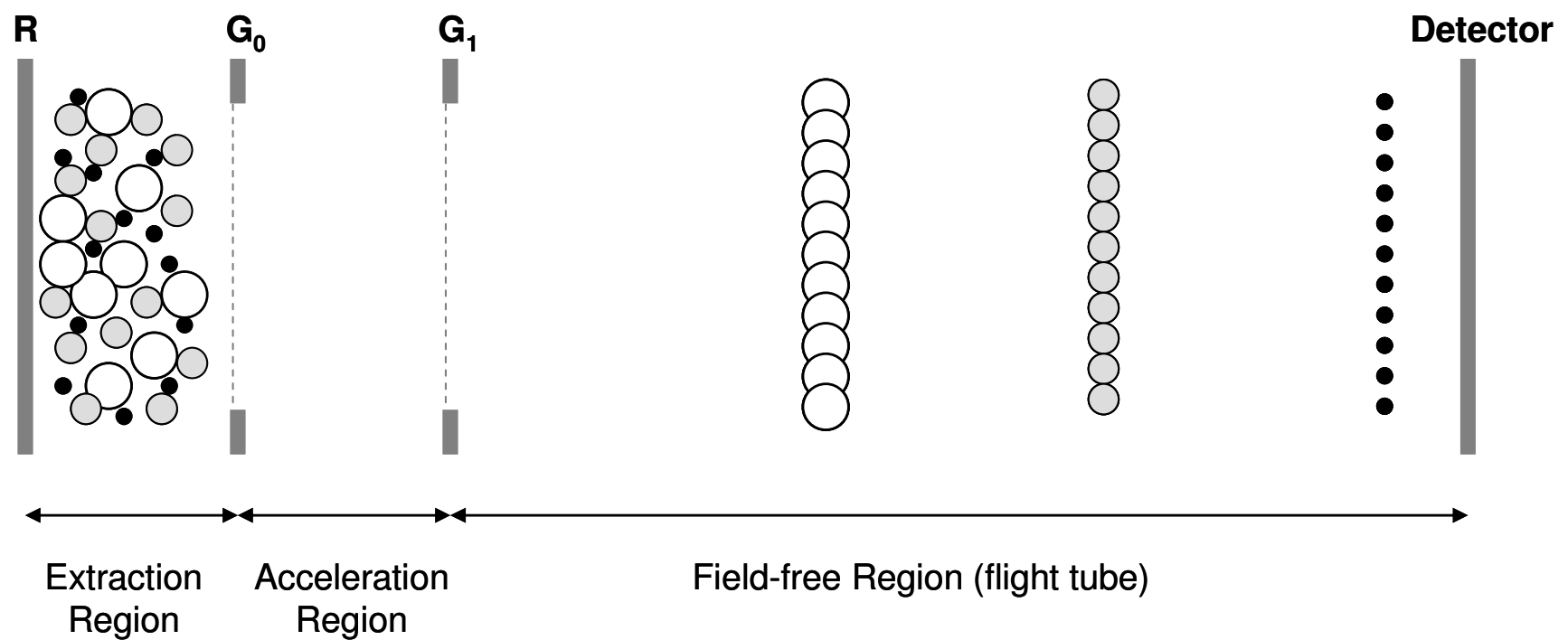

Figure 1.7. Schematic of a simplified ToFMS system depicting the mass dispersion for three ions of different $\mathrm{m} / \mathrm{z}$ with the repeller plate $(R)$, extraction grid $\left(G_{0}\right)$, and the acceleration plate $\left(G_{1}\right)$ [Adapted from 24]. 


\subsection{References}

1. D. A. Skoog, D. M. West, F. J. Holler, Fundamentals of Analytical Chemistry: Fifth Edition, Saunders College Publishing: New York (1988).

2. H. A. Laitinen, G. W. Ewing, A History of Analytical Chemistry, The Division of Analytical Chemistry of the American Chemical Society: York, PA (1977).

3. " Francis W. Aston." Nobel Web AB, Stockholm, Sweden (2007). Nobelprize.org 27May. 2007. http://nobelprize.org/

4. H. J. Svec, Mass Spectrometry - Ways and Means, A Historical Perspectus, Int. J. Mass Spectrom lon Processes, 66 (1985) 3-29.

5. J G Anderson, William Morgan and X-rays, TFA, 17 (1945) 219-221.

6. G. P. Jackson, Reducing interferences in glow discharge spectroscopies using transient operation and collision-induced dissociation, West Virginia University Libraries: Morgantown, WV (2002).

7. L. Li, Molecular speciation using pulsed glow discharge time-of-flight mass spectrometry, West Virginia University Libraries: Morgantown, WV (2004).

8. J. W. Coburn, E. Kay, A New Technique for the Elemental Analysis of Thin Surface Layers of Solids, App. Phys. Letters., 19 (1971) 350-352.

9. W. W. Harrison, C. W. Magee, Hollow Cathode Ion Source for Solids Mass Spectrometry, Anal. Chem., 46 (1974) 461-464.

10. J. Zhao, J. Zhu, D. M. Lubman, Anal. Chem., 64 (1992) 142.

11. L. Li, C. M. Barshick, J. T. Millay, A. V. Welty, F. L. King, Determination of bromine in flame-retardant plastics using pulsed glow discharge mass spectrometry, Anal. Chem., 75 (2003) 3953-3961.

12. J. Teng, C. M. Barshick, D. C. Duckworth, S. J. Morton, D. H. Smith, F. L. King, Factors influencing the quantitative determination of trace elements in soils by glow discharge mass spectrometry. Appl. Spectrosc., 49 (1995) 1361-1366.

13. C. K. Pan, F. L. King, Atomic-Emission Spectrometry Employing a Pulsed Radio-Frequency Powered Glow Discharge, App. Spectrosc., 47 (1993) 2096-2101.

14. V. Majidi, M. Moser, C. Lewis, W. Hang, F. L. King, Explicit Chemical Speciation by Microsecond Pulsed Glow Discharge Time-of-Flight Mass Spectrometry: Concurrent Acquisition of Structural, Molecular and Elemental Information, J. Anal. At. Spectrom., 15 (2000) 19-25.

15. C. L. Lewis, M. A. Moser, D. E. Dale, W. Hang, C. Hassell, F. L. King, V. Majidi, Time-gated pulsed glow discharge: Real-time chemical speciation at the elemental, structural, and molecular level for gas chromatography timeof-flight mass spectrometry. Analytical Chemistry 75 (2003) 1983-1996.

16. L. Li, J. T. Millay, J. P. Turner, F. L. King, Millisecond pulsed radio frequency glow discharge time of flight mass spectrometry: temporal and spatial variations in molecular energetics. Journal of the American Society for Mass Spectrometry 15 (2004) 87-102. 
17. R. K. Marcus, Glow Discharge Plasmas as Tunable Sources for Elemental Speciation. In R. C. Cornelis (Ed.), Elemental Speciation: Techniques and Methodology (334-355), John Wiley and Sons: London , 2003.

18. A. Bogaerts, Plasma Ionization: Glow Discharge Basics. In G. E. Tranter (Ed), J. L. Holmes (Ed), J. C. Lindon (Ed), Encyclopedia of Spectroscopy and Spectrometry (669-677), Academic Press: London, 2000.

19. B. N. Chapman, Glow Discharge Processes, John Wiley and Sons: New York (1980).

20. R. K. Marcus, Glow Discharge Spectroscopies, Plenum Press: New York (1993).

21.J. A. Kingler, P. J. Savickas, W. W. Harrison, The pulsed glow discharge as an elemental ion source, J. Am. Soc. Mass Spectrom., 1 (1990) 138-143.

22. W. O. Walden, W. W. Harrison, B. W. Smith, J. D. Winefordner, Multielement glow discharge atomic fluorescence using a continuum source, J. Anal. At. Spectrom., 9 (1994) 1039-1043.

23. A. M. Leach, D. M. McClenathan, G. M. Hieftje, Plasma Source Time-offlight Mass Spectrometry: A Powerful Tool for Elemental Speciation. In R. C. Cornelis (Ed.), Elemental Speciation: Techniques and Methodology (313333), John Wiley and Sons: London, 2003.

24. D. M. McClenathan, S. J. Ray, W. C. Wetzel, G. M. Hieftje, Plasma Source TOFMS, American Chem. Soc., 76 (2004) 159A-166A.

25. E. Hoffmann, V. Stroobant, Mass Spectrometry: Principles and Applications $2^{\text {nd }} E d$., John Wiley and Sons: West Sussex, England (2002).

26. D. Fliegel, K. Fuhrer, M. Gonin, D. Gunther, Evaluation of a pulsed glow discharge time-of-flight mass spectrometer as a detector for gas chromatography and the influence of the glow discharge source parameters on the information volume in chemical speciation analysis, Anal. Bioanal. Chem. , 386 (2006) 169-179. 


\section{Chapter 2}

Electronic Perturbation Investigations into Excitation and Ionization in the Millisecond Pulsed Glow Discharge Plasma 


\section{Abstract}

This study employed a power perturbation method to examine the energy transfer processes at different locations within the afterpeak regime of a millisecond pulsed glow discharge plasma. Brief power perturbation pulses were applied during the afterpeak regime altering the environment of the collapsing plasma. Responses of several transitions to the power perturbations were measured via atomic emission and absorption spectroscopic methods at various distances from the surface of the cathode. The experimental data provide further insight into the energy transfer processes that occur at different spatial locations and in different temporal regimes of these pulsed glow discharge plasmas. Although the enhancement of the large population of metastable argon atoms is again confirmed, the mechanism responsible for this enhancement remains unclear. The most likely possibility involves some form of ion-electron recombination followed by radiative relaxation of the resulting species. The metastable argon atoms subsequently Penning ionize sputtered copper atoms which then appear to undergo a similar ion-electron recombination process yielding variable degrees of observable afterpeak emission for copper atom transitions. The kinetic information of these processes was approximated from the corresponding relaxation time. The electron thermalization time allowing for recombination with ions was found to be $\sim 25 \mu$ s after the discharge power termination. 


\subsection{Introduction}

Glow discharges find broad use as the source of atomization, excitation, and ionization in various analytical spectrometries [1]. Sputtering, an inherent process in the normal operation of a glow discharge plasma, affords the direct atomization of analyte from solid samples, and provides a ground state atom population for analytical measurement via atomic absorption [2] and fluorescence [3] spectrometries. Subsequent excitation and ionization mechanisms in the glow discharge plasma yield excited-state atoms for determinations by atomic emission spectrometry [4] and ions for mass spectrometric determination [5].

Most analytical glow discharges operate in a continuous mode, yielding essentially steady-state analytical signals. Glow discharges operating in a pulsed power mode have been explored as an effective approach to enhance sputtering, excitation, and ionization while avoiding problems related to sample overheating and thermal degradation. In such pulsed operation, a relatively brief "power-on" period followed by a much longer "power-off" period allows for efficient sample cooling. A pulsed glow discharge with the same average power input as a continuous glow discharge is reported to yield a 100 -fold increase in signal intensity [4]. Furthermore, pulsed operation allows for much higher instantaneous power and, when coupled with time-gated detection, yields several unique analytical advantages [6]. These advantages include not only improved ionization efficiencies, but also the creation of three distinct time regimes, the prepeak, plateau, and afterpeak, during which the signal to noise ratio is enhanced. Of these three regimes, the period immediately following the discharge power termination, the afterpeak, has been the focus of recent 
attention because it affords the ability to study the unique plasma chemistry of metastable argon species.

While studying a high pressure (10-20 Torr) pulsed helium microwave discharge plasma, Johnson et al [7] observed that the total light intensity of the plasma started to rise and reached a maximum at $0.05-1 \mathrm{~ms}$ after the power termination. The intensity increase was thus termed "afterglow". A similar "afterglow" phenomenon was observed in a low-pressure glow discharge plasma by Strauss and coworkers [8]. The authors ascribed this phenomenon to excitation after power termination arising from energetic electrons released during the energy pooling (ionization) process of two metastable argon atoms.

Later, Harrison and coworkers $[9,10]$ investigated the ion species in a pulsed glow discharge plasma and observed that a large number of sputtered copper atoms were ionized after the termination of discharge power, yielding an "afterpeak" in the mass spectrometric signal. Combining mass spectrometric and spectroscopic measurements, our laboratory undertook studies of ion formation mechanisms within the afterpeak regime. These studies gave further confirmation that the population of metastable argon atoms increases after power termination and indicated that Penning ionization was responsible for the increase in analyte ion signal $[11,12,13,14]$.

Metastable atoms efficiently Penning ionize the plasma species of lower ionization potentials, generally sputtered analyte atoms. Subsequently, these analyte ions can recombine with electrons to form highly excited atoms that radiatively decay, thereby causing the emission intensity for atomic transitions of 
the analyte to increase. Because Penning ionization is limited by the potential energies of metastable argon atoms, $11.55 \mathrm{eV}$ and $11.72 \mathrm{eV}$, the afterpeak regime can provide for energy-tuned, selective ionization. For elemental analysis, this permits temporal discrimination between signals of analytes and their interferences [15]. This selective ionization has also found use in molecular mass spectrometry. The "soft" Penning ionization of the afterpeak produces principally molecular ions to provide molecular weight information for analytes. $[16,17]$

Even now, there remains some debate regarding the origin of the afterpeak. Some data indicate that the ion-electron recombination for argon is a low-probability process $[18,19]$. A theoretical modeling study done by Bogaerts [20] predicts that an increase in the metastable atom population requires a 100fold increase in thermalized electron density during the afterpeak regime. The two orders of magnitude increase, although expected to be readily detected, has not yet been experimentally observed. The spectroscopic data presented here, strongly indicate that ion-electron recombination is critical to the production of argon metastable atoms.

This work employed a perturbation method [21, 22] to examine the energy transfer processes within the afterpeak regime of a pulsed glow discharge plasma. Brief power pulses were applied during the afterpeak regime to disturb the environment of the collapsing plasma, especially electron energies. The responses of various plasma species to these "perturbation pulses" were followed using atomic emission or absorption spectroscopy. The data acquired 
provide greater insight into the fundamental plasma processes within the afterpeak regime, including further evidence of the role of ion-electron recombination. Unfortunately the exact nature of the ion-electron recombination process remains unresolved. Qualitative information regarding the kinetics of these processes is also gleaned from the observed response times.

\subsection{Experimental}

The pulsed glow discharge spectroscopy system employed in these investigations, Figure 2.1, consisted of the glow discharge source, a $0.64 \mathrm{~m}$ monochromator based optical spectroscopy system, and associated optics and electronics as described below. The operation of the system to achieve various spectroscopic measurements is also described.

\subsubsection{Glow Discharge Source}

A stainless steel six-way cross (MDC, Hayward, CA), equipped with two Suprasil optical view ports (Heraeus Quartz, Duluth, GA), served as the glow discharge chamber and the grounded anode. A vacuum, $<1 \mathrm{~m}$ Torr, was maintained in the chamber by a turbomolecular pump (Pfeiffer TPH50, Nashua, $\mathrm{NH}$ ) and a molecular pump. Ultra pure argon (Airgas, Randor, PA) was introduced through a metering valve to provide the discharge support gas. Argon pressures were monitored by a thermocouple pressure gauge (Varian 801, Lexington, MA). A copper disk cathode (NIST SRM 495, Gaithersburg, MD), 5 $\mathrm{mm}$ in diameter and $2 \mathrm{~mm}$ in thickness, was mounted on a direct insertion probe 
(DIP) equipped with a high voltage electrical feed through and introduced into the discharge chamber via a ball valve interlock.

The pulsed discharge power supply system consists of a function generator (DS345, Stanford Research Systems, Sunnyvale, CA 94089), an electronic high voltage pulser (GRX-H, Directed Energy, Fort Collins, $\mathrm{CO}$ ), and a power supply (Kepco APH 2000M, Flushing, NY). The function generator was programmed to produce the desired waveforms, which in turn triggered the pulser to gate the high voltage output from the power supply resulting in precisely controlled square wave power pulses. The function generator also provided triggers to synchronize the signal detection system. The resultant pulse trains were monitored with an oscilloscope (Tektronix 2232, Beaverton, NY) equipped with a high voltage probe (Tektronix P6015, Beaverton, NY). 


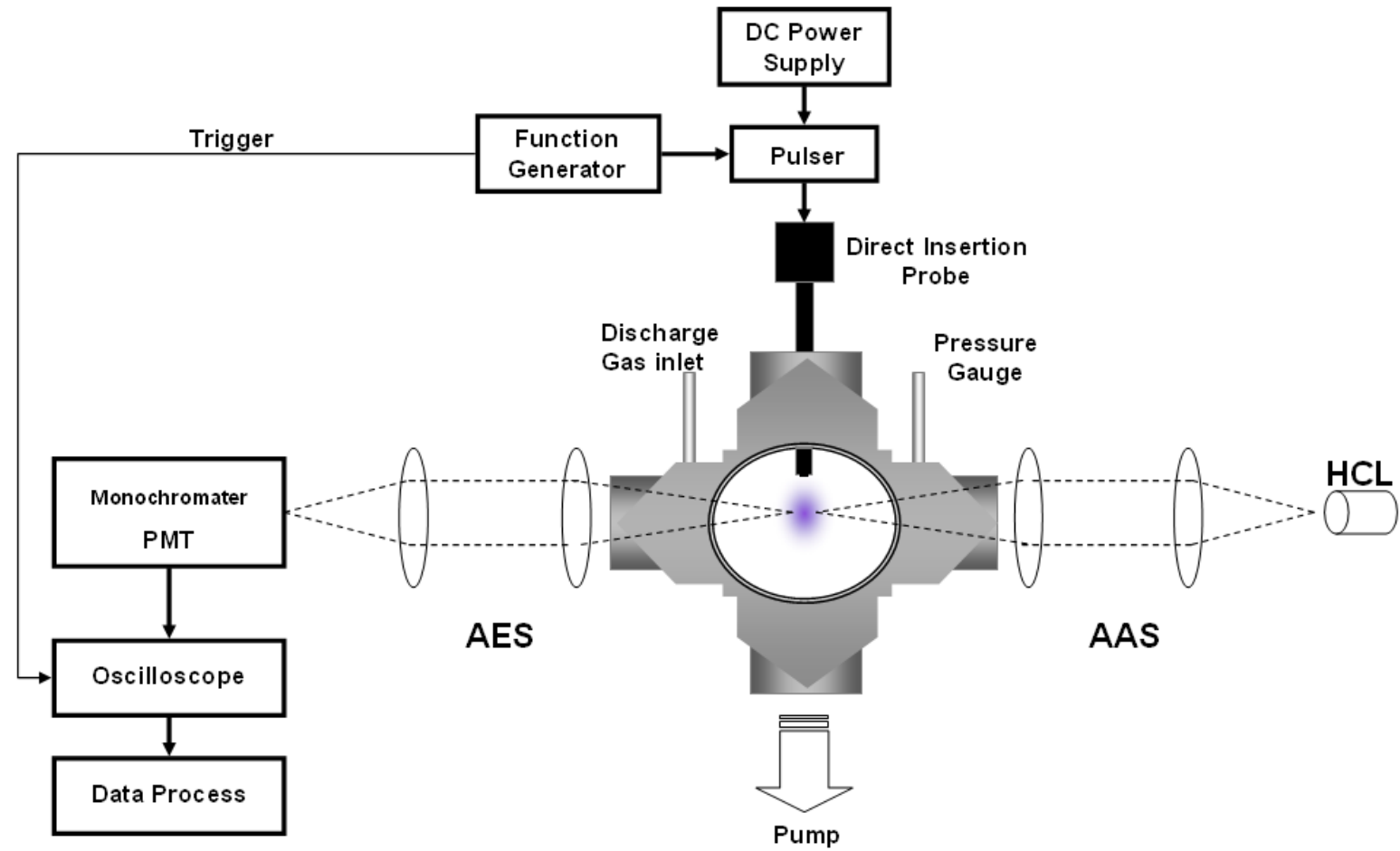

Figure 2.1. Schematic of the pulsed glow discharge spectroscopy system 


\subsubsection{Atomic Emission Measurements}

Atomic emission was used to determine the population of various excited states. Two plano-convex lenses were used to project the GD plasma image, 1:1, on the entrance of a Czerny-Turner monochromator (ISA HR-640, Edison, $\mathrm{NJ})$. The monochromator entrance and exit slits were both set at widths of 50 $\mu \mathrm{m}$ with a height of $1 \mathrm{~mm}$. Throughout this study, the lateral center of the plasma image was carefully aligned with the entrance. Variation of the position of cathode with respect to the orthogonal axis of the optical system enables measurements of the plasma at different distances from the cathode surface. The zero position was determined when the copper cathode surface image was located at the horizontal and vertical center of the entrance slit.

A photomultiplier tube (Hamamatsu R928, Bridgewater, NJ) detected the emission output of the monochromator and the signal was fed into an oscilloscope (LeCroy LT342, Chestnut Ridge, NY) that provided temporal emission profiles. The profiles were digitized using 2500 data points and averaged for 100 sweeps by the oscilloscope. The resultant data were then transferred into a spreadsheet program (Excel, Microsoft, Seattle, WA) on a computer through a GPIB interface. Further data processing and graphing was achieved using a visual basic macro in the spreadsheet.

\subsubsection{Atomic Absorption Measurements}

The populations of argon metastable atoms and ground copper atoms were measured using an atomic absorption technique. Boron-argon and copperneon hollow cathode lamps (HCL) operated at $15 \mathrm{~mA}$ current were used as the 
light sources for measurements of argon or copper species respectively. The incident light was first focused into a beam in the discharge chamber above the center of the cathode by two plano-convex lenses. The transmitted light was then imaged onto the monochromator entrance slit by the same optical lenses used in the emission measurements.

In this study, an approach was taken to eliminate the use of a lock-in amplifier and any associated temporal distortion. This very basic method relies on the stability and reproducibility of the plasma and simply involves three measurements followed by an absorbance calculation (Figure 2.2). First, the emission intensity profile of the $\mathrm{HCL}\left(\mathrm{I}_{\mathrm{HCL}}\right)$ is recorded. Then, the GD plasma is turned on and the total signal $\left(I_{\text {total }}\right)$, which consists of the emission from the GD plasma (I Ilasma $_{\text {a }}$ ) and the transmitted light from the HCL after being absorbed by the plasma $\left(I_{\text {trans }}\right)$, is recorded. Finally, the HCL light source is blocked and only the emission from the plasma ( $\left.I_{\text {plasma }}\right)$ is recorded. The differences between the total signal and the plasma emission signal are calculated to give the transmitted light intensity ( $\left.\mathrm{I}_{\text {trans }}\right)$. The absorbance is then calculated based on the wellknown, simple relationship in Equation 2.1.

$$
A=\log \left[I_{\mathrm{HCL}} / I_{\text {trans }}\right]=\log \left[\mathrm{I}_{\mathrm{HCL}} /\left(\left.\mathrm{I}_{\text {total }}\right|_{\text {plasma }}\right)\right] \quad \text { (Equation 2.1) }
$$

Where: $\mathrm{I}_{\mathrm{HCL}}=$ emission intensity of the HCL.

$I_{\text {trans }}=$ intensity of transmitted light.

$I_{\text {plasma }}=$ emission intensity of the plasma only.

$I_{\text {total }}=$ total radiation intensity of both transmitted light and plasma emission. 
Because the absorbance profiles were calculated from emission signals there is no temporal distortion arising from the lock-in amplifier time constant. The resultant absorbance profiles can hence be directly compared with the emission profiles in terms of temporal characteristics (Figure 2.2). 


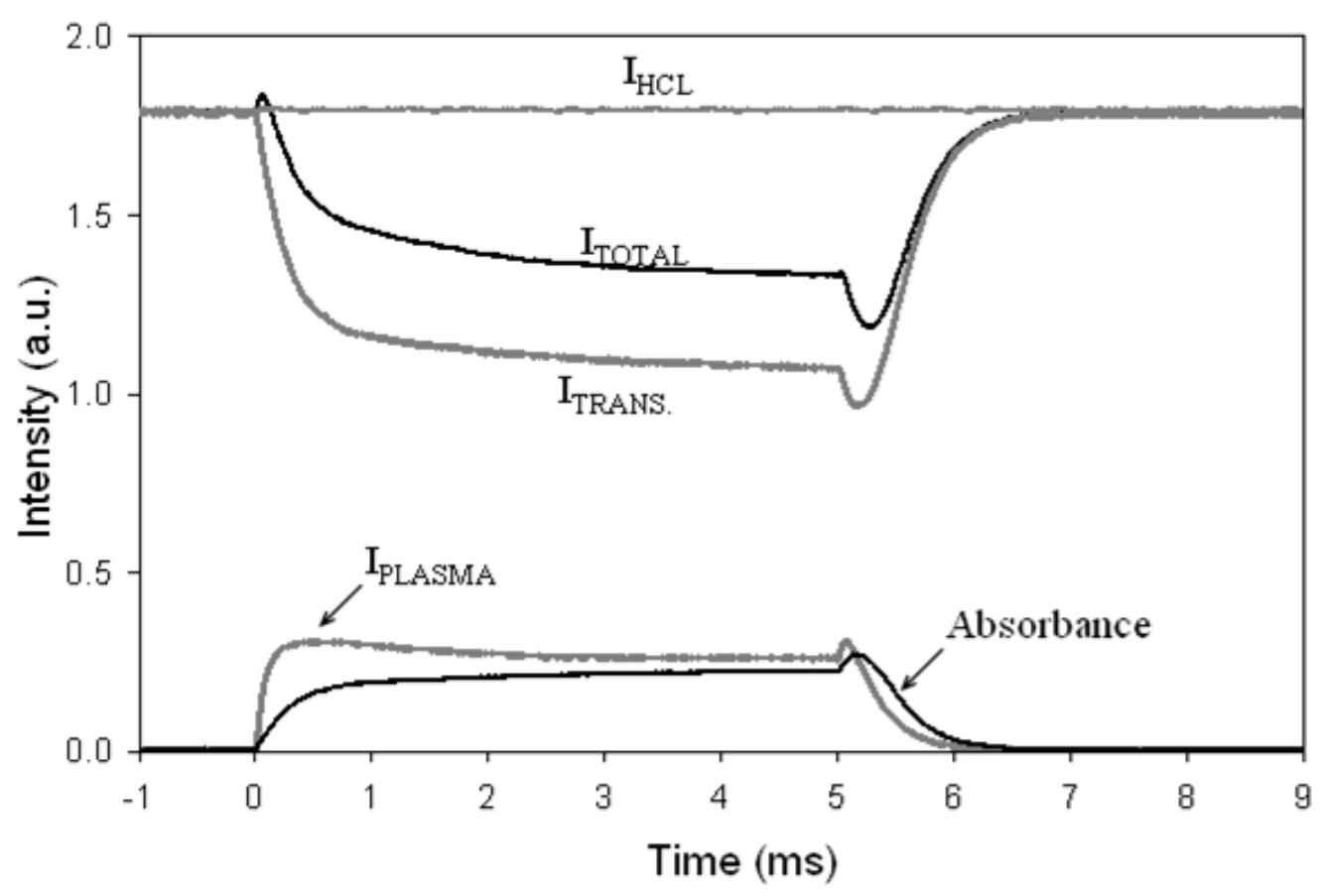

Figure 2.2. The temporal profile of measured HCL emission intensity ( $\left.\mathrm{I}_{\mathrm{HCL}}\right)$, plasma emission intensity (IPLASMA), total emission intensity (ITOTAL), and the calculated transmitted light intensity (ITRANS.) and absorbance. 


\subsubsection{Power Modulation to Yield a Perturbation Pulse}

The pulsed GD plasma was operated under a fixed pressure at 0.8 Torr. Under the normal pulse mode, the discharge power was kept on for $5 \mathrm{~ms}$ and turned off for $15 \mathrm{~ms}$, giving a $50 \mathrm{~Hz}$ pulse rate with a $25 \%$ duty cycle (Figure 2.3a). The pulse voltage was set at $800 \mathrm{~V}$ and the peak current was measured to be $3.2 \mathrm{~mA}$, resulting in a 0.64 Watt average power.

In order to explore the plasma processes within the afterpeak regime, brief power pulses were applied shortly after the termination of the power pulse to perturb the collapsing plasma (Figure $2.3 \mathrm{~b}$ and $3 \mathrm{c}$ ); the brief pulse is therefore termed a "perturbation pulse". The perturbing pulses had the same magnitude and power as the original pulse, differing only in the length of their on-time. The duration of the perturbation pulse was set for $0.1 \mathrm{~ms}$ and was positioned at 0.1 $\mathrm{ms}$ after power termination for the single perturbing pulse, and at $0.1 \mathrm{~ms}$ and 0.3 ms after power termination for the double perturbing pulses. It should be noted that increasing the length of the perturbing pulse proportionately increases the maximum intensity until a plateau, steady state discharge, is reached. In another study, the position of the single perturbing pulse was varied from 0.2 to $1.0 \mathrm{~ms}$ after the termination of the discharge plasma. 


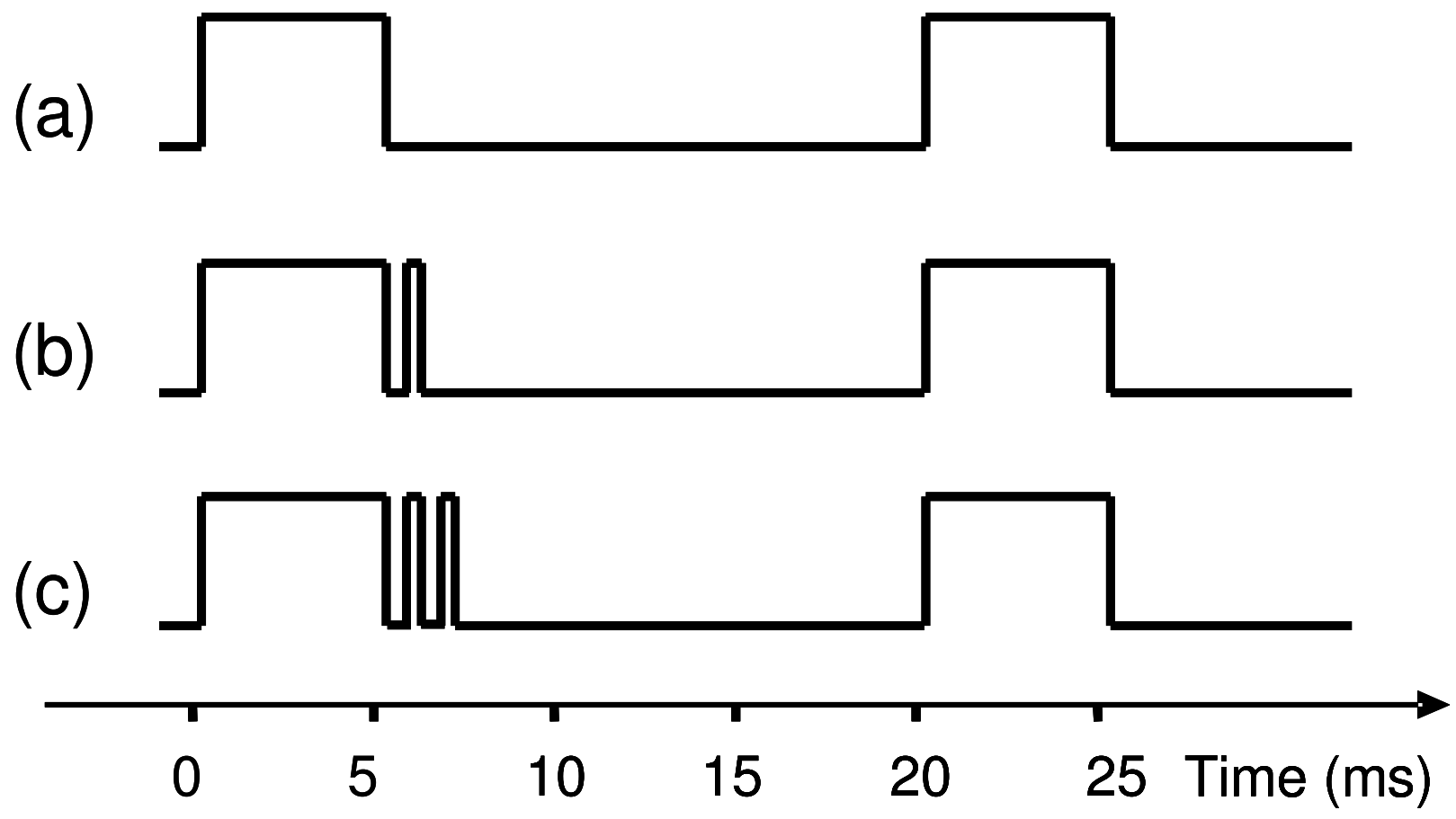

Figure 2.3. The temporal profiles of the applied power of (a) the normal pulsed glow discharge; (b) the pulsed glow discharge with a single perturbation pulse applied during the afterpeak $0.1 \mathrm{~ms}$ following power termination; and (c) two perturbation pulses applied 0.1 and $0.3 \mathrm{~ms}$ following termination. Profiles are not drawn to scale. 


\subsection{Results and Discussion}

An applied perturbation pulse generates an instantaneous electric field between the anode and cathode. Among the plasma species, electrons are most sensitive to transient shifts in the electric field. The electric field immediately energizes free electrons because of their light mass and higher mobility. These energetic electrons are then able to excite or ionize the heavier particles, i.e. atoms and molecules, via collisions. These excited or ionized plasma species can further dissipate their energies to the surrounding environment through various processes. The energy transfer chain can be schematically presented as follows:

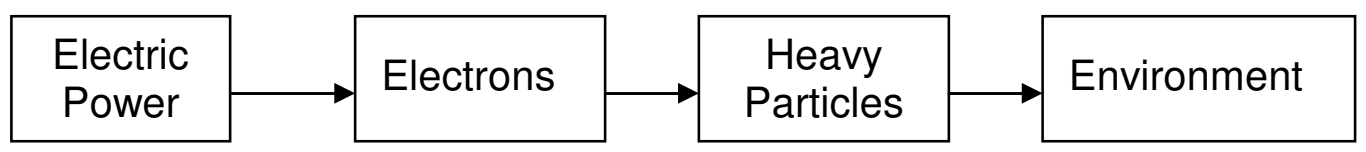

An important channel for this energy dissipation process in excited species is the emission of photons via radiative relaxation. When the power is terminated, the electron population rapidly thermalizes [23, 24]. Highly energetic electrons disappear from the plasma rapidly and thermal equilibrium occurs $\leq$ $100 \mu$ s after the power termination [24], suspending electron excitation and ionization.

Because of the close energy coupling, electronic perturbation of the plasma leads to changes in the spectroscopic observables of the plasma. Therefore, the study of the impact of these electric field induced electronic perturbations on spectral signals provides additional insight into the energy 
transfer processes in the plasma. A side benefit is that the temporal profiles also contain information regarding the kinetics of these energy transfer processes.

In this study, temporal spectroscopic signal profile responses of discharge gas and sputtered species to perturbation pulse modulations were monitored. The transitions explored are listed in Table 2.1; of each group, a representative transition will be discussed in detail.

Table 2.1. Selected spectral lines and the respective transitions.

\begin{tabular}{|c|c|c|}
\hline Group & Wavelength (nm) & Transition \\
\hline Argon atom & $\begin{array}{l}\text { Ar I } 811.5 \\
\text { Ar I } 794.8 \\
\text { Ar I } 415.8 \\
\text { Ar I } 420.0 \\
\text { Ar I 810.3 } \\
\text { Ar | 750.3 }\end{array}$ & $\begin{array}{l}4 p 2-4 s{ }^{3} P_{2} \\
4 p 7-4 s{ }^{3} P_{0} \\
5 p 5-4 s{ }^{3} P_{2} \\
5 p 2-4 s{ }^{3} P_{2} \\
4 p 4-4 s{ }^{3} P_{1} \\
4 p 10-4 s{ }^{1} P_{1}\end{array}$ \\
\hline Argon ion & $\begin{array}{l}\text { Ar II } 476.5 \\
\text { Ar II } 427.7 \\
\text { Ar II } 410.3 \\
\text { Ar II } 413.1 \\
\end{array}$ & $\begin{array}{l}4 p{ }^{2} P_{3 / 2}-4 s^{2} P_{1 / 2} \\
4 p^{\prime} P_{3 / 2}-4 s^{2} D_{5 / 2} \\
5 s^{4} P_{5 / 2}-4 p^{4} D_{7 / 2} \\
3 d^{\prime}{ }^{2} D_{3 / 2}-4 s^{\prime}{ }^{2} D_{3 / 2}\end{array}$ \\
\hline Copper atom & $\begin{array}{l}\text { Cu I } 324.7 \\
\text { Cu I } 327.4 \\
\text { Cu I } 809.3 \\
\text { Cu I } 368.7 \\
\text { Cu I } 515.3\end{array}$ & $\begin{array}{l}4 p^{2} P_{1 / 2}-4 s^{2} S_{1 / 2} \\
4 p^{3} P_{3 / 2}-4 s^{2} P_{1 / 2} \\
5 s^{2} S_{1 / 2}-4 p{ }^{2} P_{3 / 2} \\
6 d^{2} D_{5 / 2}-4 p^{2} P_{3 / 2} \\
4 d^{2} D_{3 / 2}-4 p^{2} P_{1 / 2}\end{array}$ \\
\hline
\end{tabular}

Note: The transitions in bold are discussed in detail as the representative for each group. 


\subsubsection{Argon Ion Emission}

Because all of the argon ion transitions exhibit similar emission responses, the response of the transition from $4 p^{2} \mathrm{P}_{3 / 2}(19.87 \mathrm{eV})$ to $4 s^{2} \mathrm{P}_{1 / 2}(17.26 \mathrm{eV})$, yielding emission at $476.5 \mathrm{~nm}$, is discussed as representative of this group. Excited argon ions are most likely produced by a two-step process: electron ionization of argon followed by electron excitation [14].

1) electron ionization: $\mathrm{Ar}+\mathrm{e}^{-}$(fast) $\rightarrow \mathrm{Ar}^{+}+2 \mathrm{e}^{-}$(slow)

2) electron excitation $\mathrm{Ar}^{+}+\mathrm{e}^{-}$(fast) $\rightarrow \mathrm{Ar}^{+}$(excited) $+\mathrm{e}^{-}$(slow)

Both steps directly involve energetic electrons; therefore, the emission is expected to exhibit immediate responses to power modulation. Indeed, the spontaneous responses can be readily recognized in Figure 2.4a: the emission intensities quickly decay as soon as the power is turned off. As explained earlier, energetic electrons disappear immediately after the power termination and both ionization and excitation halt; therefore, the excited argon ion state is no longer populated. Consequently, the emission undergoes a sharp decrease. Following the same argument, the application of the perturbation pulse will reverse the processes and the intensities instantaneously increase (Figures 2.4b and 4c). 

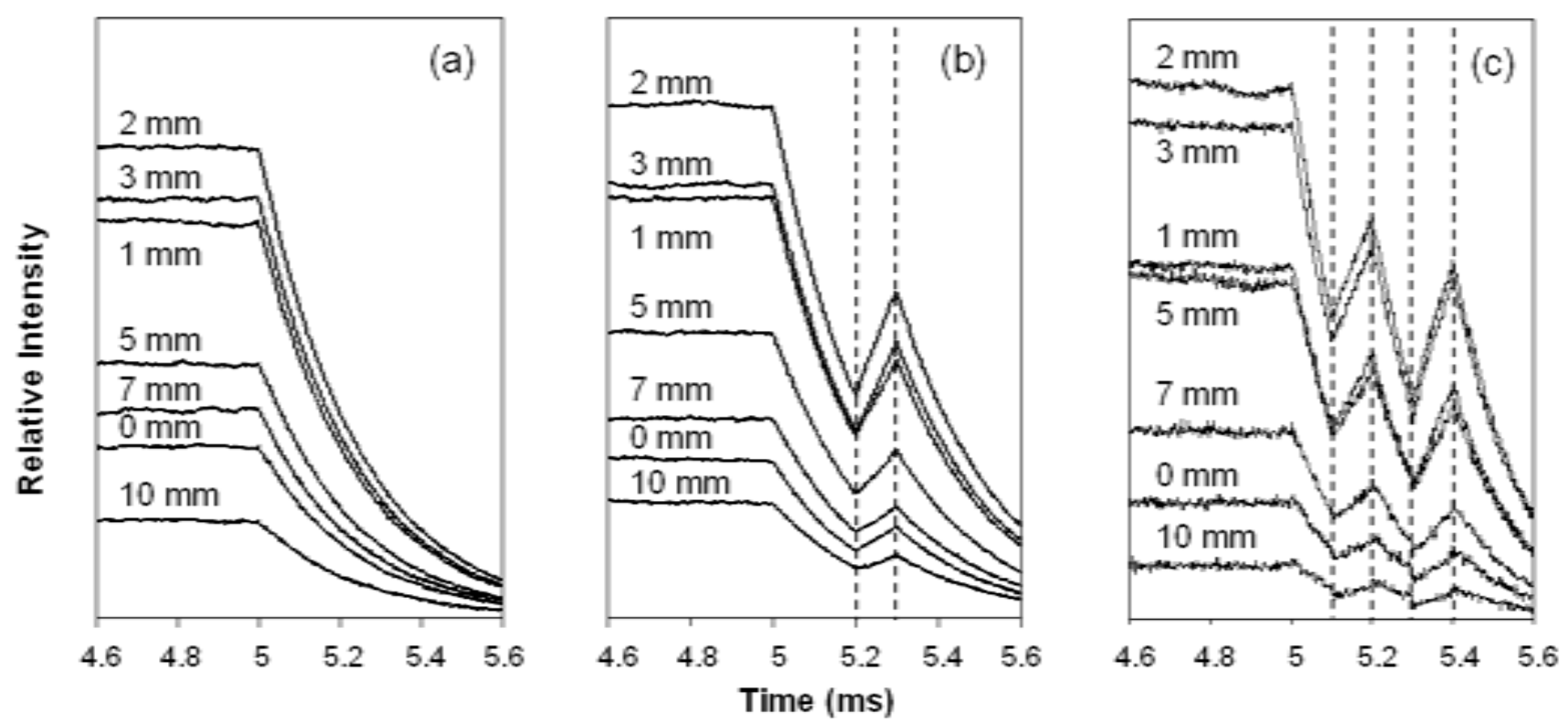

Figure 2.4. The temporal profiles of Ar II $476.5 \mathrm{~nm}$ emission at various distances: (a) the normal pulsed glow discharge; (b) the pulsed glow discharge with the perturbation pulse applied from $5.2 \mathrm{~ms}$ to $5.3 \mathrm{~ms}$; and (c) the pulsed glow discharge with the double perturbation pulse applied from 5.1 to $5.2 \mathrm{~ms}$ and 5.3 to $5.4 \mathrm{~ms}$. 


\subsubsection{Argon Atom Emissions}

Excited argon atoms can be produced through various mechanisms in a glow discharge. Within the cathode dark space $(0-2 \mathrm{~mm})$, fast atom excitation is the dominant excitation mechanism [25] because of the presence of the strong electric field.

1) Energy coupling into the argon ion:

Electric Field $+\mathrm{Ar}^{+}$(slow) $\rightarrow \mathrm{Ar}^{+}$(fast)

2) Charge exchange yielding fast atoms:

$$
\mathrm{Ar}^{+} \text {(fast) }+\operatorname{Ar}(\text { slow }) \rightarrow \mathrm{Ar}^{+}(\text {slow })+\operatorname{Ar} \text { (fast) }
$$

3) Fast atom excitation:

$$
\operatorname{Ar}(\text { fast })+\operatorname{Ar} \rightarrow \operatorname{Ar}(\text { slow })+\operatorname{Ar}^{*} \text { (excited) }
$$

In the negative glow region $(3-10 \mathrm{~mm})$, the major excitation mechanisms are electron excitation and ion-electron recombination.

1) Electron excitation:

$$
\mathrm{e}^{-} \text {(fast) }+\operatorname{Ar}(\text { ground }) \rightarrow \mathrm{e}^{-}(\text {slow })+A r^{*}(\text { excited })
$$

2) Ion-electron recombination-decay:

$$
\mathrm{Ar}^{+}+\mathrm{e}^{-}(\text {slow }) \rightarrow \mathrm{Ar}^{* *} \text { (highly excited) }
$$

The highly excited argons lie close to the ionization continuum; once formed, they radiatively decay to lower excited states: $\mathrm{Ar}^{\star \star} \rightarrow \mathrm{Ar}^{\star}+$ photon. 
Figure 2.5 shows the temporal profiles of argon atom emissions at 811.5 $\mathrm{nm}$. This emission corresponds to the transition from $4 \mathrm{p}^{3} \mathrm{D}_{3}(13.08 \mathrm{eV})$ to the $4 \mathrm{~s}$ ${ }^{3} \mathrm{P}_{2}$ metastable state $(11.55 \mathrm{eV})$. It is evident that the temporal profiles are spatially dependent. In the close proximity of the cathode surface $(0-1 \mathrm{~mm})$, the emissions quickly decay after the power termination; no afterpeak is observed. However, once the perturbation pulse is applied the intensities start to enhance. These observations are consistent with the proposed fast atom excitation mechanism within the cathode dark space. Note that the mechanism is closely coupled with electric field that is essential for the production of fast atoms. The power termination, i.e., disappearance of the electric field, immediately suspends this mechanism. Hence, the cessation of the power halts fast atom bombardment excitation resulting in near immediate intensity attenuation. When the perturbation pulse is switched on, the electric field is re-established; fast atom bombardment excitation is re-initiated and, therefore, the emission signals start to increase. 

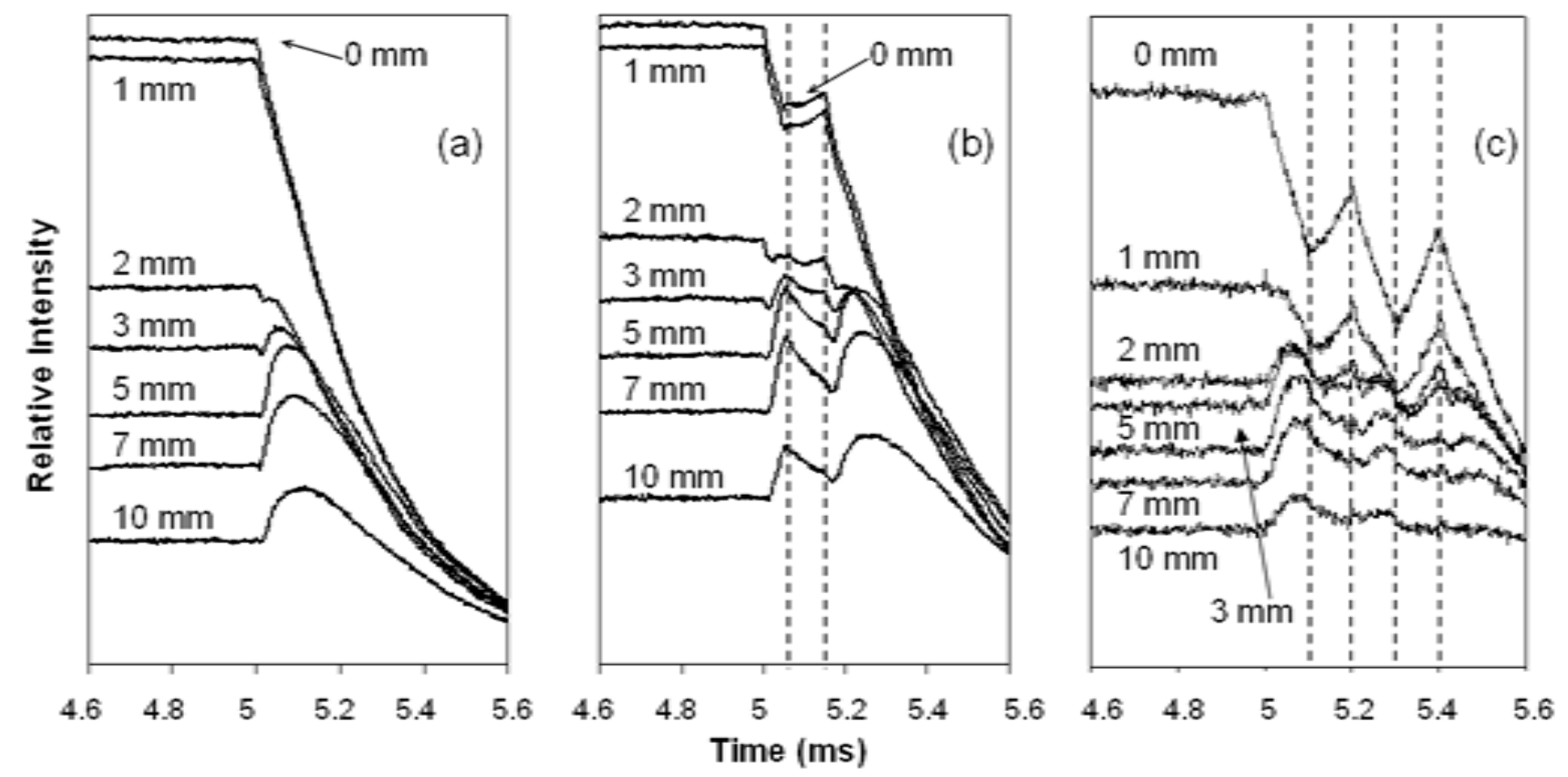

Figure 2.5. The temporal profiles of Ar I $811.5 \mathrm{~nm}$ emission at various distances for (a) the normal pulsed glow discharge; (b) the pulsed glow discharge with the perturbation pulse applied from 5.05 to $5.15 \mathrm{~ms}$; and (c) the pulsed glow discharge with the double perturbation pulse applied from 5.1 to $5.2 \mathrm{~ms}$ and 5.3 to $5.4 \mathrm{~ms}$ (within the dashed lines). 
At distances between 3 to $10 \mathrm{~mm}$, which lie within the negative glow region, the emission exhibits completely different responses. The intensities increase after the power termination and form the so-called "afterpeaks". The intensity upsurges, or the increases in excited argon atom population, arise from either electron excitation or ion-electron recombination-decay.

In this case, the plasma perturbation is especially useful in allowing one to determine what type of excitation mechanism is dominant. The electron excitation and ion-electron recombination have completely opposite responses to plasma perturbation. The power application increases the average electron energy and also allows for electron excitation to be further enhanced. On the other hand, as the electrons are accelerated, the thermalized electron population diminishes and the probability of ion-electron recombination is lowered. Therefore, if electron excitation is the dominant mechanism, the $811.5 \mathrm{~nm}$ emission intensity would exhibit a positive response to the applied perturbation pulse; that is, the intensity would increase at the onset of the perturbation pulse. Otherwise, a negative response would strongly suggest ion-electron recombination-decay to be the dominant excitation mechanism.

The experimental results, shown in Figures $2.5 \mathrm{~b}$ and c, clearly show that the intensities undergo an apparent decrease at the onset of the perturbation pulse. Therefore, ion-electron recombination-decay is the predominant mechanism populating the argon excited state during the afterpeak regime. As expected, the intensities start to increase after the perturbation pulse is terminated and ion-electron recombination re-establishes. It is evident that the 
intensity increases are delayed with respect to power termination. Because ionelectron recombination requires that the electron be thermalized, the delays are ascribed to the electron thermalization time. Based on the time difference between the power termination and the resurgence of emission intensity, the electron thermalization time is estimated to be $\sim 100 \mu \mathrm{s}$, in agreement with Biondi [24]. However, in this study, the thermalization time cannot be explicitly determined because of signal distortion.

\subsubsection{Argon Metastable Atom Absorption}

The metastable atoms of discharge gas play very important roles in excitation and ionization; therefore, the population of the metastable atoms and their production mechanism are of special interest. In this study, the population of the argon ${ }^{3} \mathrm{P}_{2}$ metastable state was monitored by measuring the absorbance at $811.5 \mathrm{~nm}$ and the results are shown in Figure 2.6.

The temporal responses of argon metastable atom ${ }^{3} \mathrm{P}_{2}$ population are found to be correlated with the respective argon atom emission profiles. Within the cathode dark space ( 0-1 mm, Figures 2.6a, b, and c), the metastable population decreases as the discharge power pulse is turned off and then immediately increases at the onset of the perturbation pulse. The observations suggest that, as with the other excited states, the metastable state is also predominantly populated by fast atom excitation inside the cathode dark space.

The afterpeaks were observed at approximate distances of 3-10 mm (Figures 2.6d, e, and f), indicating that metastable atoms continue to form within 
the negative glow region after the power termination. As discussed earlier, the dominant excitation mechanism is ion-electron recombination followed by radiative decay. When the perturbation pulse is applied, electrons are energized and ion-electron recombination is suppressed. In addition, the energetic electrons deconstruct metastable atoms via electron ionization and excitation, further depopulating the metastable state. Therefore, the metastable atom population undergoes a sudden decrease (Figure 2.6d).

After the termination of the perturbation pulse, the argon metastable population starts to recover. The recovery is delayed with respect to the perturbation pulse termination. Note that the delay closely matches the emission signal delay observed in Figure 2.5b, i.e., the assumed electron thermalization time. That is, the argon metastable population starts to increase as soon as the argon ion-electron recombination-decay re-initiates. The strong correlation provides additional evidence for the conclusion that the recombination-decay mechanism is responsible for the formation of metastable atoms. 


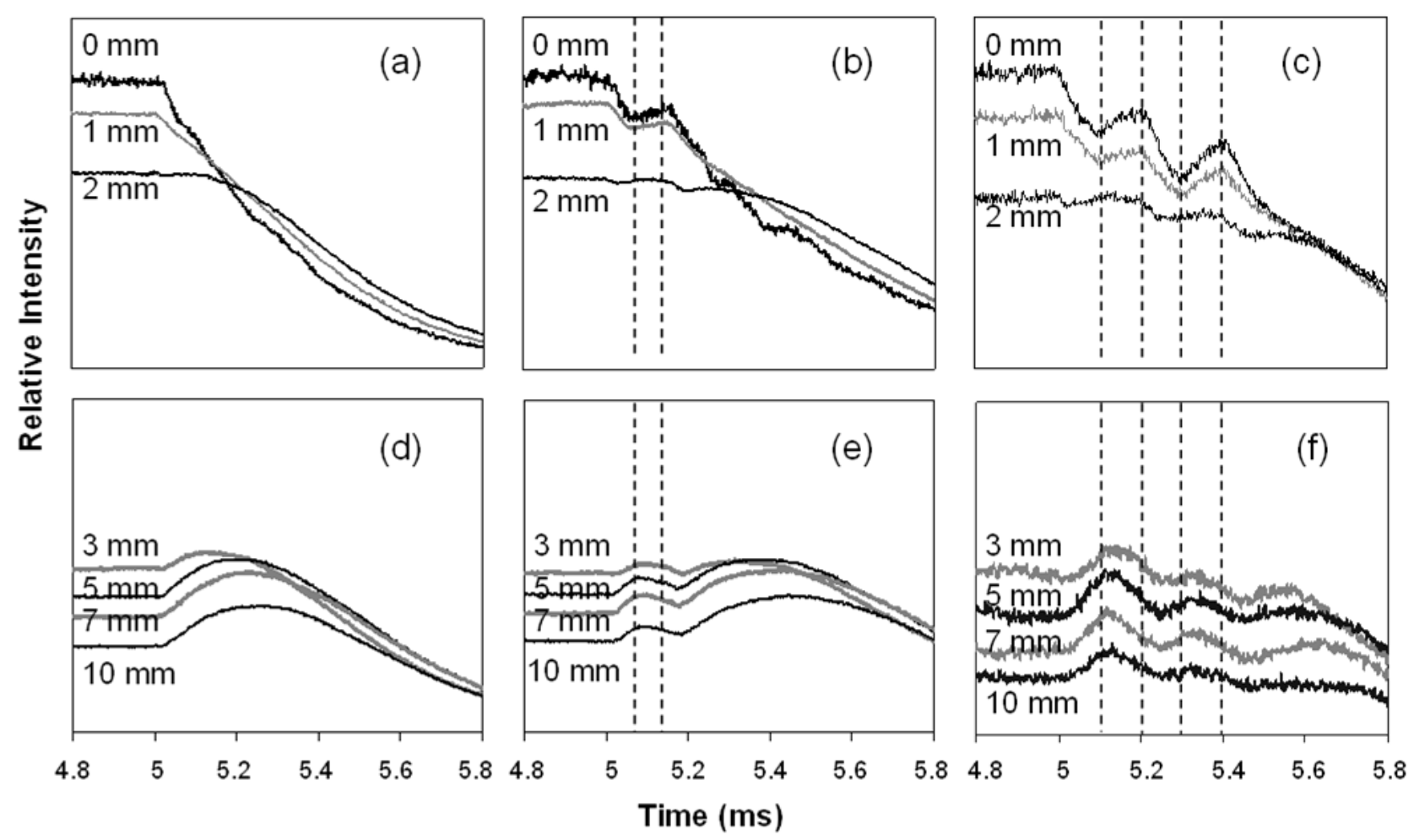

Figure 2.6. The temporal profile of argon metastable atom absorbance at $811.5 \mathrm{~nm}$ for (a) the normal pulsed glow discharge at 0-2 $\mathrm{mm}$ and (d) 3-10 $\mathrm{mm}$; (b) the pulsed glow discharge with the perturbation pulse applied from 5.05 to $5.15 \mathrm{~ms}$ (within the dashed lines) at $0-2 \mathrm{~mm}$ and (e) $3-10 \mathrm{~mm}$; and (c) the double perturbation applied from 5.1 to $5.2 \mathrm{~ms}$ and 5.3 to 5.4 ms (within the dashed lines). 


\subsubsection{Copper Atom Emission}

The excited $\mathrm{Cu}$ atom group is represented by the emission at $368.7 \mathrm{~nm}$ which originates from the $6 d^{2} D_{5 / 2}$ state $(7.18 \mathrm{eV})$. Significant afterpeaks were found throughout the plasma after the power was terminated (Figure 2.7a). Atomic absorption measurements of copper revealed that the copper atom population in the plasma remains relatively stable during the same period because of the slow diffusion processes (Figure 2.8). Therefore, the afterpeaks can be readily attributed to the corresponding excitation mechanisms of copper.

Strauss et al. [8] also observed similar intensity increases and ascribed the increases to electron excitation. The energy pooling of a pair of argon metastable atoms yields an electron carrying the extra energy of $7.48 \mathrm{eV}$. The energetic electrons generated can subsequently excite copper atoms.

$$
\begin{aligned}
& \mathrm{Ar}^{\mathrm{m}}+\mathrm{Ar}^{\mathrm{m}} \rightarrow \mathrm{Ar}^{0}+\mathrm{Ar}^{+}+\mathrm{e}^{-}+\Delta \mathrm{E} \text { where } \Delta \mathrm{E}=7.48 \mathrm{eV} \\
& \mathrm{e}^{-} \text {(fast) }+\mathrm{Cu}^{0} \text { (ground) } \rightarrow \mathrm{Cu}^{*} \text { (Excited) }
\end{aligned}
$$

An alternative mechanism is similar to the recombination-decay of argon [14]. The increasing argon metastable atoms Penning ionize copper atoms directly, resulting in an upsurge of copper ions after power termination $[4,10]$. The resultant copper ions recombine with thermalized electrons and subsequently populate the excited states of copper atoms through radiative cascade decay.

$$
\begin{aligned}
& \mathrm{Ar}^{\mathrm{m}}+\mathrm{Cu}^{0} \rightarrow \mathrm{Ar}+\mathrm{Cu}^{+} \\
& \mathrm{Cu}^{+}+\mathrm{e}^{-} \text {(slow) } \rightarrow \mathrm{Cu}^{*} \text { (excited) }
\end{aligned}
$$


Again, the dominant excitation mechanism can be determined by applying the perturbation method. Figures $2.7 \mathrm{~b}$ and $\mathrm{c}$ show the responses of the copper emission to the applied perturbation pulse. The onset of perturbation pulses causes sudden and significant decreases in the intensities at various distances; the intensities start to increase after the pulse is terminated. However, unlike the argon excitation emissions, no spatial differences are observed. The negative responses strongly suggest that copper atoms are predominantly excited through an ion-electron recombination-decay mechanism throughout the plasma. Shortly after the perturbation pulse is terminated, the copper emission intensities undergo a resurgence. Also observed in argon excited atom emissions, delays of $\sim 100 \mu$ s can be discerned for the resurgences with respect to the perturbation pulse termination; these delays are assigned to electron thermalization time and the values are consistent with the previous measurement.

Close examination reveals that the copper emission afterpeaks maximize at $\sim 5.2 \mathrm{~ms}$. As compared to the argon emissions, the maxima are delayed for $\sim 0.1 \mathrm{~ms}$, suggesting that the copper ion-electron recombination follows argon ion-electron recombination in time. Furthermore, the maximum positions and the breadths of the copper afterpeaks closely match those of the argon metastable population afterpeaks shown in Figures 2.6e and $\mathrm{f}$. This suggests that the excited copper atoms are associated with argon metastable atoms: the copper ion population that undergoes further recombination-decay arises from Penning ionization during the afterpeak regime, rather than from the residual copper ions in the plateau regime. 

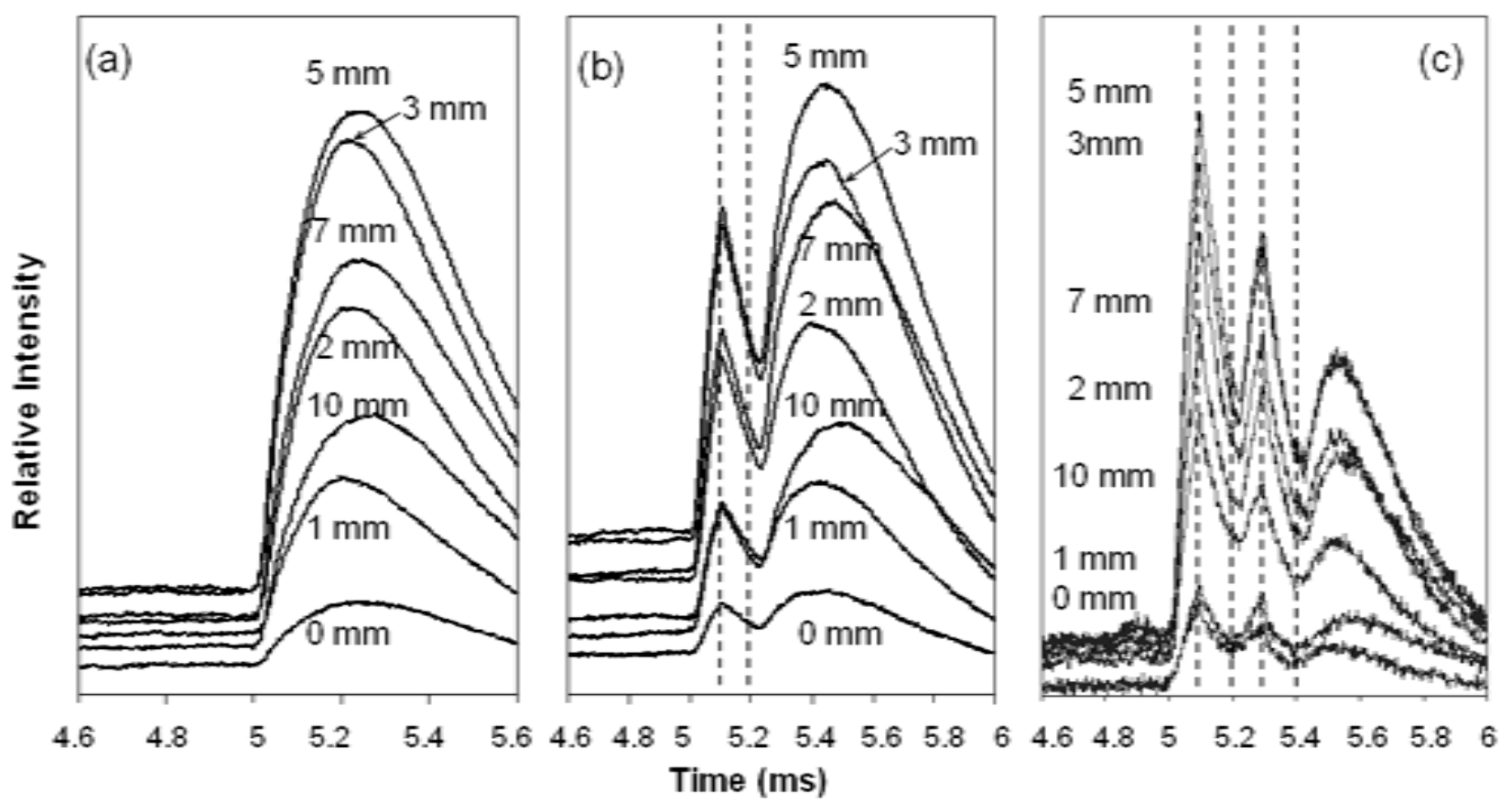

Figure 2.7. The temporal profiles of $\mathrm{Cu} I 368.7 \mathrm{~nm}$ emission at various distances for (a) the normal pulsed glow discharge; (b) the pulsed glow discharge with the perturbation pulse applied from 5.1 to $5.2 \mathrm{~ms}$; the pulsed glow discharge with the double perturbation pulse applied from 5.1 to $5.2 \mathrm{~ms}$ and 5.3 to $5.4 \mathrm{~ms}$. 


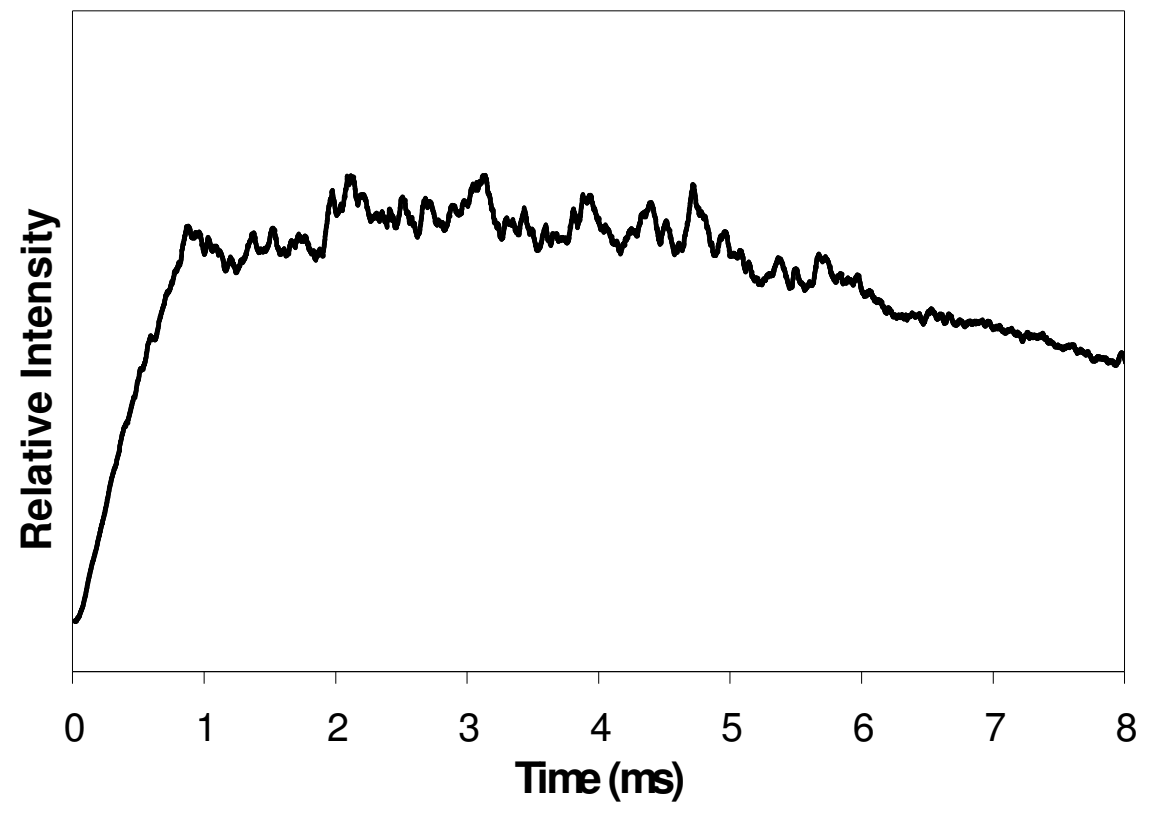

Figure 2.8. A typical temporal profile of copper atom absorbance signal at $334.7 \mathrm{~nm}$ in a pulsed glow discharge (sample distance: $4 \mathrm{~mm}$ ). 


\subsubsection{Variation of the Perturbation Pulse in Time}

A study was undertaken to examine the effect of varying the position of the perturbing pulse in time. The position of the perturbing pulse relative to power termination was varied from 0.1 to $1.0 \mathrm{~ms}$. The glow discharge plasma was observed within both the dark space and negative glow region with cathode distances of $0 \mathrm{~mm}$ and $5 \mathrm{~mm}$ respectively. As stated in section 2.2, the lateral center of the plasma image was carefully aligned with the entrance. The zero position was determined when the copper cathode surface image was located at the horizontal and vertical center of the entrance slit. The temporal profiles of the two positions can be seen in Figures 2.9a, b, c and d.

As seen in section 2.2, in close proximity to the cathode surface (Figure 2.9a) the emissions quickly decay after the termination of power and are enhanced once the perturbing pulse is applied. Again this observation is in agreement with the mechanism of fast atom excitation thought to be dominant within the cathode dark space. At distances farther from the cathode surface, in this case $5 \mathrm{~mm}$ (Figure 2.9b, c, and d), emission intensities increase following power termination due to ion-electron recombination-decay to form the afterpeak. With the onset of a perturbation pulse soon after power termination, about $0.1 \mathrm{~ms}$ (Figures $2.9 \mathrm{c}$ and d), the emission intensities quickly drop and then rise again with the pulse's cessation. However, the introduction of perturbation pulses set farther in time from the termination of power, 0.2 to $1.0 \mathrm{~ms}$ (Figure 2.9b), causes a spike in intensity followed by a mini-afterpeak once the pulse has ended. 
Within the cathode dark space (Figure 2.9a), the position of the perturbing pulse relative to the termination of power seems to have little effect on both the shape and intensity of the resulting upsurge. However, within the negative glow region of the plasma (Figure 2.9b), as the perturbing pulse is moved out farther in time, the intensity of the emission upsurge following the cessation of the perturbing pulse decreases. This is most likely due to lower populations of metastable argon atoms at times farther from the termination of power. Similar results were also observed for Ar II $476.5 \mathrm{~nm}$ emissions which have no afterpeak, and $\mathrm{Cu}$ I $368.7 \mathrm{~nm}$ emissions with an afterpeak. 

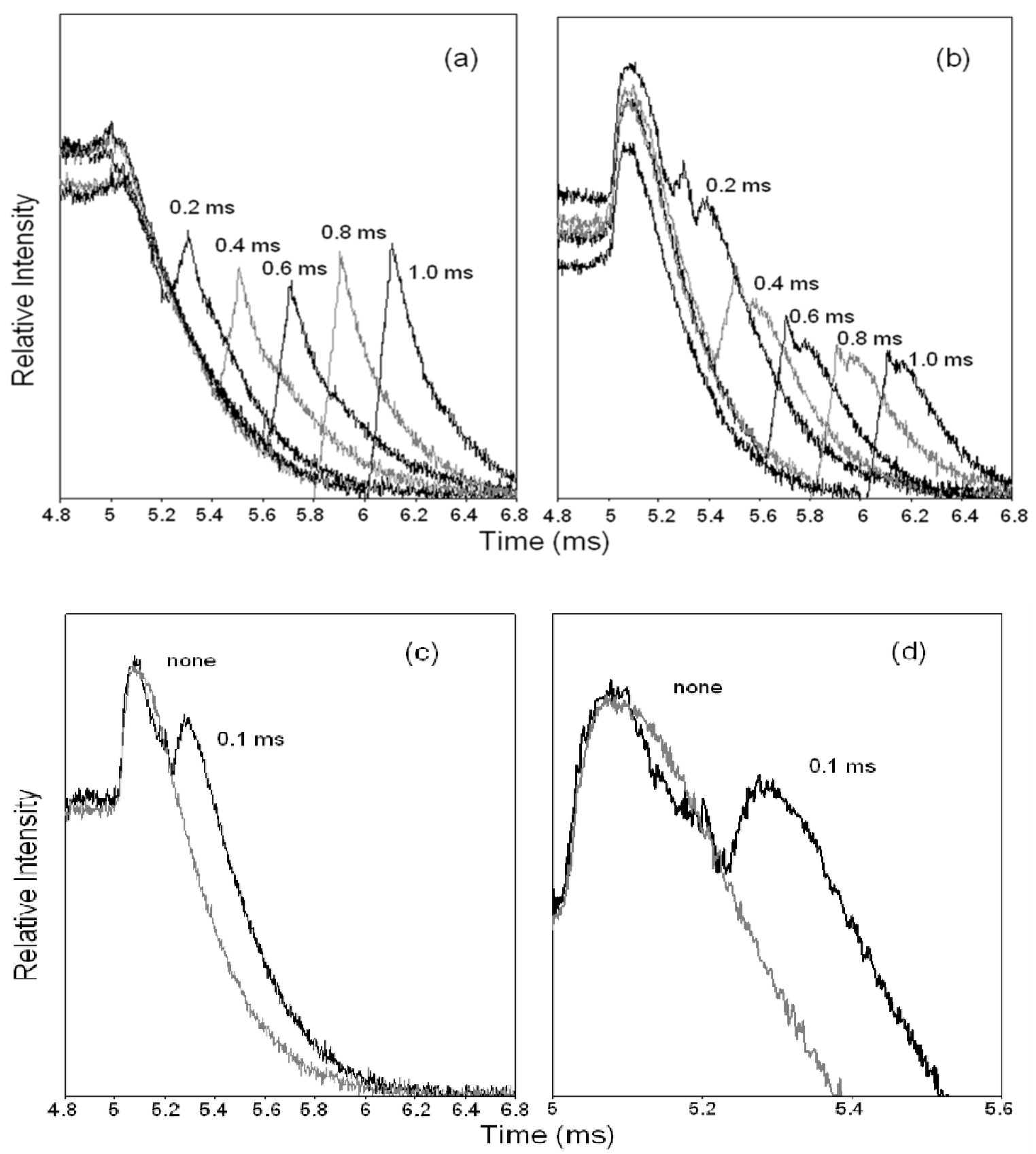

Figure 2.9. The temporal profiles of Ar I $811.5 \mathrm{~nm}$ emission with the perturbing pulse at various times $(0.2,0.4,0.6,0.8$, and $1.0 \mathrm{~ms})$ after power termination with the cathode positioned at (a) $0 \mathrm{~mm}$ with no afterpeak; and (b) $5 \mathrm{~mm}$ with an afterpeak. Figure (c) also has the cathode positioned at $5 \mathrm{~mm}$ and shows profiles with $(0.1 \mathrm{~ms})$ and without the perturbing pulse. Figure (d) is a close-up of (c). 


\subsection{Conclusions}

This study employed a perturbation method to probe the ionization and excitation processes within the afterpeak regime of a pulsed glow discharge plasma. The responses of various plasma species to the perturbation were measured using spectroscopic techniques. The experiments strongly suggest the following processes take place in sequence after the power termination.

1) electron thermalization;

2) argon ion-electron recombination-decay forming argon metastable atoms;

3) Penning ionization of copper atoms yielding copper ions;

4) copper ion-electron recombination-decay forming excited copper atoms.

Through these processes, the plasma energy cascades from the argon ion $(>15 \mathrm{eV})$ to the argon metastable state $(>11.55 \mathrm{eV})$, to the copper ion $(7.7 \mathrm{eV})$, and then the excited copper atom. The downward energy flow arises from the plasma decay after the power input has halted. Since the radiative decay of argon metastable states is forbidden, these states serve as energy reservoirs where their populations undergo temporary increases during the decay process. Consequently, the energetic (11.55 and $11.72 \mathrm{eV})$ metastable atoms play a central role in ionization and excitation during the afterpeak regime. It is important to note that the exact identity of the ion-electron recombination process remains unclear, particularly given that there does not appear to be an increase in electron density during the afterpeak that current theory indicates is required. 
Further experiments to identify the responsible ion-electron recombination mechanism are planned.

The experiments also revealed valuable information regarding the kinetics of the associated plasma processes, such as electron thermalization time. However, the signal distortion associated with the detection system prevents accurate measurements of these values. Future work will update the signal detection system to make accurate measurements. 


\subsection{References}

1. W. W. Harrison, C. Barshick, J. A. Kingler, P. H. Ratcliff, Y. Mei, Glow discharge techniques in analytical chemistry, Anal. Chem., 62 (1990) 943A949A.

2. C. L. Chakrabarti, K. L. Headrick, J. C. Hutton, Z. Bicheng, P. C. Bertels, M. $\mathrm{H}$. Back, Pulsed and transient nodes of atomization by cathodic sputtering in a glow discharge for atomic absorption spectroscopy, Anal. Chem., 62 (1990) 574-586.

3. W. O. Walden, W. W. Harrison, B. W. Smith, J. D. Winefordner, Multielement glow discharge atomic fluorescence using a continuum source, $J$. Anal. At. Spectrom., 9 (1994) 1039-1043.

4. C. Pan, F. L. King, Atomic emission spectroscopy employing a pulsed radiofrequency powered glow discharge, Appl. Spectrosc., 47 (1993) 2096-2101.

5. F. L. King, W. W. Harrison, Glow discharge mass spectrometry: an introduction to the technology and its utility, Mass Spectrom. Rev., 9 (1990) 285-317.

6. R. E. Steiner, C. L. Lewis, F. L. King, Tim-of-Flight mass spectrometry with a pulsed glow discharge ionization source, Anal. Chem., 69 (1997) 17151721.

7. R. A. Johnson, B. T. McClure, R. V. Holt, Electron removal in the helium afterglow, Phys. Rev., 80 (1950) 376-379.

8. J. A. Strauss, N. P. Ferreira, H. G. C. Human, An investigation into the role of the metastable argon atoms in the afterglow of a low-pressure discharge, Spectrochim. Acta, 37B (1982) 947-954.

9. J. A. Kingler, P. J. Savickas, W. W. Harrison, The pulsed glow discharge as an elemental ion source, J. Am. Soc. Mass Spectrom., 1 (1990) 138-143.

10. J. A. Kingler, C. M. Barshick, W. W. Harrison, Factors influencing ion signal profiles in pulsed glow discharge mass spectrometry, Anal. Chem., 63 (1991) 2571-2576.

11. F. L. King, C. Pan, Temporal signal profiles of analyte species in modulated glow discharge plasmas, Anal. Chem., 65 (1993) 735-739.

12. C. Pan, F. L. King, lon formation processes in the afterpeak time regime of pulsed glow discharge plasmas, J. Am. Soc. Mass Spectrom., 4 (1993) 727732.

13. G. P. Jackson, C. L. Lewis, S. K. Doorn, V. Majidi, F. L. King, Spectral, spatial, and temporal characteristics of a millisecond pulsed glow discharge: metastable argon atom production, Spectrochim. Acta, 56B (2001) 24492464.

14. C. L. Lewis, G. P. Jackson, S. K. Doorn, V. Majidi, F. L. King, Spectral, spatial, and temporal characteristics of a millisecond pulsed glow discharge: copper analyte emission and ionization, Spectrochim. Acta, 56B (2001) 487-501. 
15. C. L. Lewis, E. S. Oxley, C. Pan, F. L. King, Determination of $40 \mathrm{Ca}^{+}$in the presence of $40 \mathrm{Ar}^{+}$: an illustration of the utility of time-gated detection in pulsed glow discharge mass spectrometry, Anal. Chem., 71 (1999) 230234.

16. V. Majidi, M. Moser, C. Lewis, W. Hang, F. L. King, Explicit chemical speciation by microsecond pulsed glow discharge Time-of-Flight mass spectrometry: concurrent acquisition of structural, molecular, and elemental information, J. Anal. At. Spectrom., 15 (2000) 19-25.

17. R. E. Steiner, C. L. Lewis, V. Majidi, Consideration of a millisecond pulsed glow discharge Time-of-Flight mass spectrometry for concurrent elemental and molecular analysis, J. Anal. At. Spectrom., 14 (1999) 1537-1541.

18. D. R. Bates, Dissociation Recombination, Phys. Rev., 78(1950) 492-493.

19. D. R. Bates, Electron Recombination in helium, Phys. Rev., 77 (1950) 718719.

20. A. Bogaerts, R. Gijbels, G. P. Jackson, Modeling of a millisecond pulsed glow discharge: investigation of the Afterpeak, J. At. Anal. Spectrom., 18 (2003) 533-548.

21. E. L. Bydder, G. P. Miller, A relaxation method for determining state of equilibrium and temperature ratio $\mathrm{Te} / \mathrm{Tg}$ in an argon ICPT, Spectrochim. Acta, 43B (1988) 819-829.

22. F. H. A. G. Fey, W. W. Stoffels, J. A. M. van der Mullen, Instantaneous and delayed responses of line intensity to interruption of radiofrequency power in an argon inductively coupled plasma, Spectrochim. Acta, 46B (1991) 885-900.

23. Y. B. Golubovskii, H. Lange, V. A. Maiorov, I. A. Porokhova, V. P. Sushkov, On the decay of metastable and resonance Xe atoms in the afterglow of a constricted discharge diffusion, J. Phys. D, 36 (2003) 694-703.

24. M. A. Biondi, de-excitation, and ionization cross sections for metastable atoms. I, Phys. Rev., 88 (1952) 660.

25. D. A. Scott, A. V. Phelps, Excitation by fast atoms at very high electric field to gas-density ratios in argon, Physical Review A, 43 (1991) 3043-3056. 


\section{Chapter 3}

Spatial, Spectral, and Temporal Characteristics of a Millisecond Pulsed Glow Discharge: Electron Density \& Temperature 


\begin{abstract}
Time-resolved Langmuir probe studies were undertaken to elucidate the excitation and ionization processes in a direct current pulsed glow discharge plasma. Electron densities and temperatures were derived from the Langmuir probe results. Attention was paid to differences observed between the power-on, plateau, and power-off, afterpeak, regimes. The data suggest that ion-electron recombination plays a much less significant role in the formation of afterpeak emissions than previously thought. Instead, based on recent work by others, it is proposed here that dissociative recombination between molecular argon ions $\left(\mathrm{Ar}_{2}{ }^{+}\right)$and electrons is the principal process responsible for emission enhancements and increases in electron temperature found within the afterpeak regime. Spatially-resolved Langmuir probe studies were also undertaken and enabled the analysis of electron densities and temperatures within the plasma as a function of axial and vertical distance from a direct insertion copper cathode. Simultaneous spectral data of several copper lines was collected using a monochromator to ascertain any affects the Langmuir probe might have on the plasma.
\end{abstract}




\subsection{Introduction}

Plasma research was pioneered in the early 1920's by Irving Langmuir, a renowned American physicist and chemist who coined the term plasma because the ionized gases reminded him of blood plasma [1]. In 1928, Langmuir described the fourth state of matter as follows: "Except near the electrodes, where there are sheaths containing very few electrons, the ionized gas contains ions and electrons in about equal numbers so that the resultant space charge is very small. We shall use the name plasma to describe this region containing balanced charges of ions and electrons" [2]. Over the past 80 years, this branch of science has developed into a major discipline with vast bodies of research spanning the fields of chemistry, physics, and astronomy. Today, key driving forces behind plasma research include their use for controlled thermonuclear fusion, for thin film deposition and etching on microelectronics, and as solid state ion sources for atomic spectroscopy and mass spectrometry [3, 4, 5]. Glow discharge plasma is just one example that has been drawing increased interest over the last two decades. Well-known for their efficient generation of atomic populations from both conducting and non-conducting solid samples via cathodic sputtering, glow discharge (GD) plasmas find broad use as sources of atomization, excitation, and ionization in various analytical spectrometries $[6,7]$.

GD plasmas are rich in ionization mechanisms, with the most dominant processes being electron ionization, charge transfer, and Penning ionization. When operated in the pulsed mode, spatial and temporal separation of the different ionization mechanisms becomes possible through the creation of three distinct time regimes during each on/off cycle: a prepeak dominated by electron 
ionization, an afterpeak dominated by Penning ionization, and a steady-state plateau which arises from a mixture of electron impact, charge transfer, and Penning ionizations [8]. Although pulsed GD has been actively researched over the past 15 years, it remains a relatively new science with much debate about its basic plasma parameters and processes, including the origin of the afterpeak. Therefore, characterization of the energies and populations of charged species present would provide greater insight into the fundamental plasma processes within the pulsed GD.

Irving Langmuir's electrostatic probe, dubbed the Langmuir probe, remains one of the simplest and most widely used plasma diagnostic devices used in the field today. The device consists of a small conducting electrode, typically tungsten or graphite, inserted into the plasma from which localized electron and ion currents can be measured at its tip [9]. By applying a voltage ramp to the probe, the response current drained from the plasma can be recorded at different probe bias voltages, generating an I-V characteristic curve [10]. The I-V characteristic curve can then be used to determine several basic properties of the plasma, including temperature and density [11]. Part of the popularity of this method is due to the probe's exceedingly simple design, ease of use, and low cost [12]. Moreover, other techniques for calculating electron temperature and density, such as optical methods utilizing excitation temperature or $\mathrm{H}_{\beta}$ line broadening, preclude most low pressure plasmas because they are not at local thermal equilibrium (LTE) and do not have sufficient electron number densities [13]. 
Several studies have used Langmuir probes to investigate the nature of charged species in if and dc GD plasmas [6, 10, 14-17]. In their investigation of a dc argon GD plasma operated in the continuous mode, Fang and Marcus found that the electron number density was proportional to the discharge current with the majority of the electron temperatures falling between $0.20-0.35 \mathrm{eV}$ and electron densities on the order of $10^{11} \mathrm{~cm}^{-3}[6]$. Heintz and Hieftje made Langmuir probe measurements for a conventional rf GD (operated in both the continuous and pulsed mode) and a if planar-magnetron source. The conventional if GD exhibited temperatures between $2-5 \mathrm{eV}$ and electron densities between $10^{9}-$ $10^{10} \mathrm{~cm}^{-3}[16]$. To our knowledge, there have not been any studies to date that have investigated pulsed dc glow discharges using the Langmuir probe method.

In the present work, time-resolved Langmuir probe studies were undertaken to determine several fundamental plasma parameters in a direct current pulsed argon glow discharge plasma. Electron densities and temperatures were derived from the Langmuir probe results and attention was paid to differences observed between the power-on, plateau, and power-off, afterpeak, regimes. Spatially-resolved Langmuir probe studies enabled the analysis of electron densities and temperatures within the plasma as a function of axial and vertical distance from a direct insertion copper cathode. Simultaneous spectral data of several copper lines was also collected using a monochromator to ascertain any affects the Langmuir probe might have on the plasma. 


\subsection{Experimental}

The pulsed glow discharge spectroscopy system employed in these investigations, Figure 3.1, consisted of the glow discharge source, a single Langmuir probe with graphite electrode, a $1.0 \mathrm{~m}$ monochromator based optical spectroscopy system, and associated optics and electronics as described below.

\subsubsection{Glow Discharge Source}

The glow discharge source is described in detail in Chapter 2 section 2.2.1 and is described only briefly here. A vacuum, $<1 \mathrm{~m}$ Torr, was maintained in the chamber and Ultra pure argon (Airgas, Randor, PA) was introduced through a metering valve to provide the discharge support gas. A copper disk cathode (NIST SRM 495, Gaithersburg, MD), $5 \mathrm{~mm}$ in diameter and $2 \mathrm{~mm}$ in thickness, was mounted on a direct insertion probe (DIP) equipped with a high voltage electrical feed through and introduced into the discharge chamber via a ball valve interlock. Between runs, the copper cathode disks and ceramic shields were polished clean, rinsed with methanol, and allowed to dry in air.

The pulsed discharge power supply system consists of a function generator (DS345, Stanford Research Systems, Sunnyvale, CA 94089), an electronic high voltage pulser (GRX-H, Directed Energy, Fort Collins, $\mathrm{CO}$ ), and a power supply (Kepco APH 2000M, Flushing, NY). The function generator was programmed to produce the desired waveforms, which in turn triggered the pulser to gate the high voltage output from the power supply resulting in precisely controlled square wave power pulses that were monitored with an oscilloscope (Tektronix 2232, Beaverton, NY). 
The pulsed dc GD plasma was operated under a fixed pressure at 0.75 torr. Under pulsed mode, the discharge power was kept on for $5 \mathrm{~ms}$ and turned off for $15 \mathrm{~ms}$, giving a $50 \mathrm{~Hz}$ pulse rate with a $25 \%$ duty cycle. The pulse voltage was set between 600-900 V and the peak current was measured to be between $.6-.1 \mathrm{~mA}$, resulting in average powers between .36 and .90 Watts.

\subsubsection{Langmuir Probe Construction}

The Langmuir probe consisted of a $0.90 \mathrm{~mm}$ diameter graphite electrode (Pentel, Torrence, CA) soldered to a brass BNC pin and insulated in a ceramic MACOR $®$ shield (Accuratus, Washington, N.J.). The electrode was attached to the BNC connector using Teflon tape. Approximately $5 \mathrm{~mm}$ of the graphite was left exposed to sample the charged particles in the glow discharge plasma, resulting in a net exposed surface area of $1.48 \times 10^{-5} \mathrm{~m}^{2}$. A schematic of the Langmuir probe assembly is shown in Figure 3.2.

The Langmuir probe was inserted into the glow discharge source using a $\mathrm{BNC}$ to $\mathrm{BNC}$ connector flange and two spacer flanges connected on one side of the six-way cross described earlier. The Langmuir probe assembly was mounted perpendicular to the cathode in the GD chamber. Vacuum integrity was maintained using O-rings between each flange. The graphite electrode was joined through the BNC connectors to a programmable dc power supply (1251DM, Kepco, Flushing, NY) from which the power ramp, -10 to $40 \mathrm{~V}$, for the I-V characteristic curves was supplied. The Langmuir probe was electrically floated between the DC power supply and the oscilloscope. The voltage drop across a 1 
$\mathrm{k} \Omega$ resistor was used to measure the response current drained from the plasma.

A schematic of the glow discharge source design with the mounted Langmuir probe and simplified circuit depicting the electrically-floating probe is shown in Figures 3.1 and 3.3. 


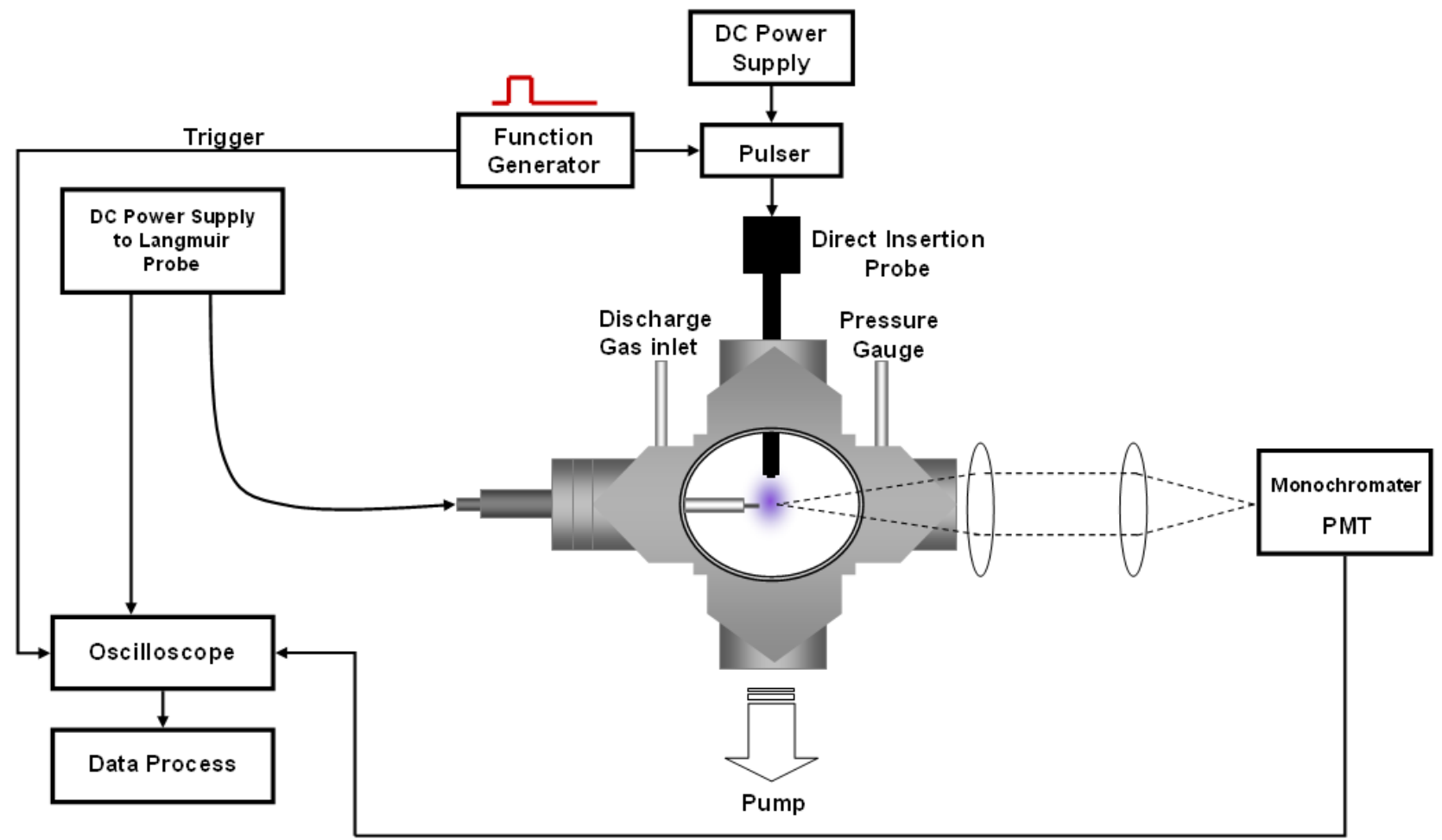

Figure 3.1. Schematic of the pulsed glow discharge spectroscopy system with Langmuir probe and monochramator. 

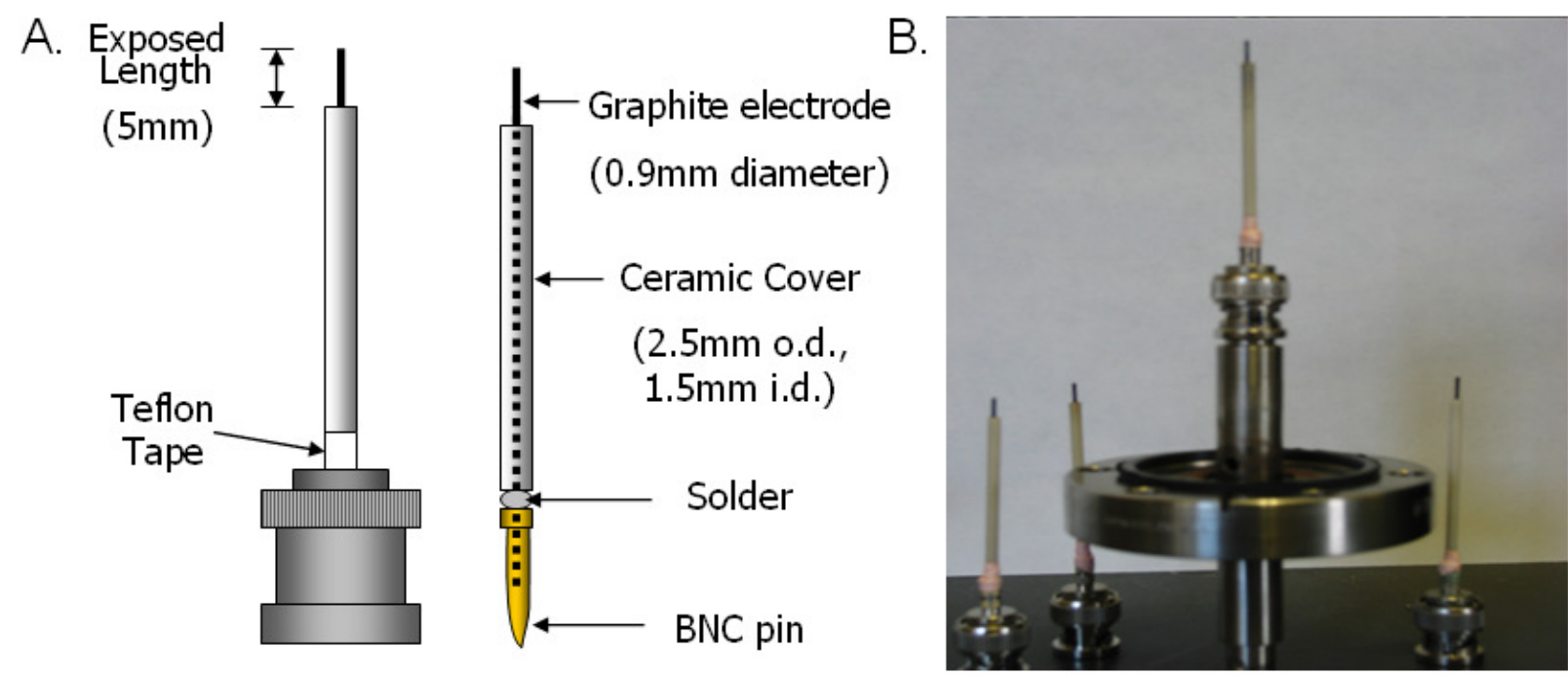

Langmuir probe with BNC to BNC connector flange

Figure 3.2. A) Schematic of the graphite Langmuir probe assembly and B) a picture of the actual Langmuir probes and the BNC to BNC connector flange used to introduce the probes into the glow discharge source. 


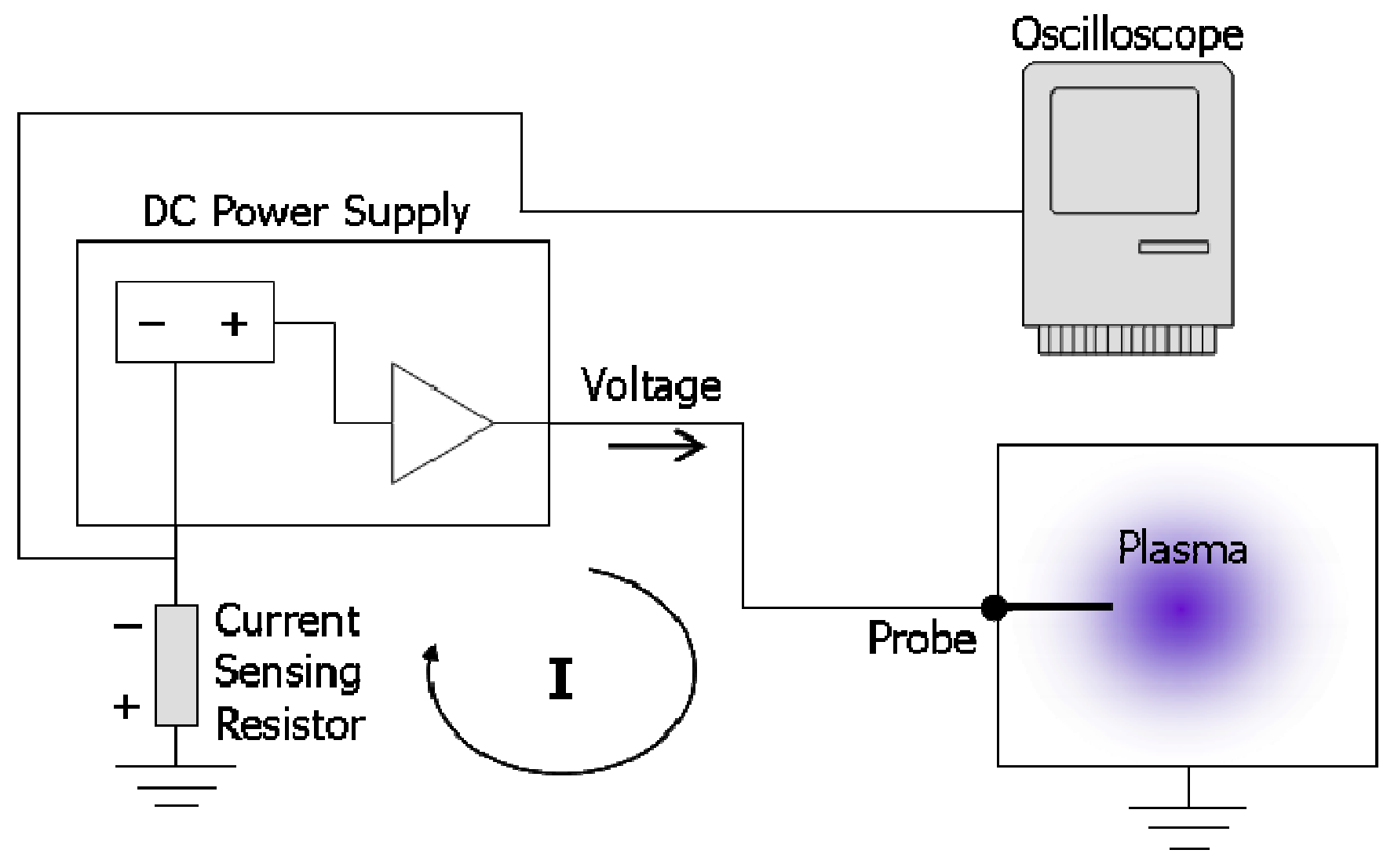

Figure 3.3. Schematic of the single Langmuir probe electrical circuit [adapted from 18]. 


\subsubsection{Langmuir Probe I-V Characteristic Measurements}

Before each data set, the copper cathode was sputtered for at least 15 minutes at the chosen dc voltage. This pre-sputtering period allows the plasma and discharge voltage to stabilize while burning off any impurities on the surface of the cathode. Because the Langmuir probe becomes coated with sputtered sample while in the plasma, ultimately affecting the conductivity and sheath formation of the graphite electrode, the cathode was situated at the farthest position ( $25 \mathrm{~mm}$ ) during each pre-sputtering period.

Once the plasma was stabilized, the cathode was moved to the desired location ( $5 \mathrm{~mm}$ from the tip of the Langmuir probe) and the I-V characteristics were measured. This was achieved by applying a manual voltage ramp (-10 to $40 \mathrm{~V}$ ) to the Langmuir probe and collecting the corresponding current flow through the probe. The pulse profiles from each response current were digitized using 10,000 data points and averaged for 100 sweeps by the oscilloscope (Figure 3.5). The resultant data were then transferred into a spreadsheet program (Excel, Microsoft, Seattle, WA) on a computer through a GPIB interface. Further data processing and graphing was achieved using a visual basic macro in the spreadsheet. To combat irregularities due to electrode coating by sputtered material and ensure comparable results, the Langmuir probes were cleaned with dilute nitric acid and rinsed with methanol between each data set. 


\subsubsection{Spectral Measurements}

To ascertain any affects the Langmuir probe might have on the plasma, simultaneous spectral data of several copper lines was collected using a monochromator. Two plano-convex lenses were used to project the GD plasma image, 1:1, on the entrance of a Czerny-Turner monochromator (ISA JY-38, Edison, NJ). The monochromator entrance and exit slits were both set at widths of $50 \mu \mathrm{m}$ with a height of $1 \mathrm{~mm}$. Throughout this study, the lateral center of the plasma image was carefully aligned with the entrance. A photomultiplier tube (Hamamatsu R928, Bridgewater, NJ) detected the emission output of the monochromator and the signal was fed into the oscilloscope described in section 3.2.3 to provide temporal emission profiles. The profiles were digitized using 2500 data points and averaged for 100 sweeps by the oscilloscope. The resultant data were then transferred into a spreadsheet program (Excel, Microsoft, Seattle, WA) on a computer through a GPIB interface. Further data processing and graphing was achieved using a visual basic macro in the spreadsheet. Figure 3.6 shows the placement of the monochromator PMT and lenses relative to the GD source and Langmuir probe. 


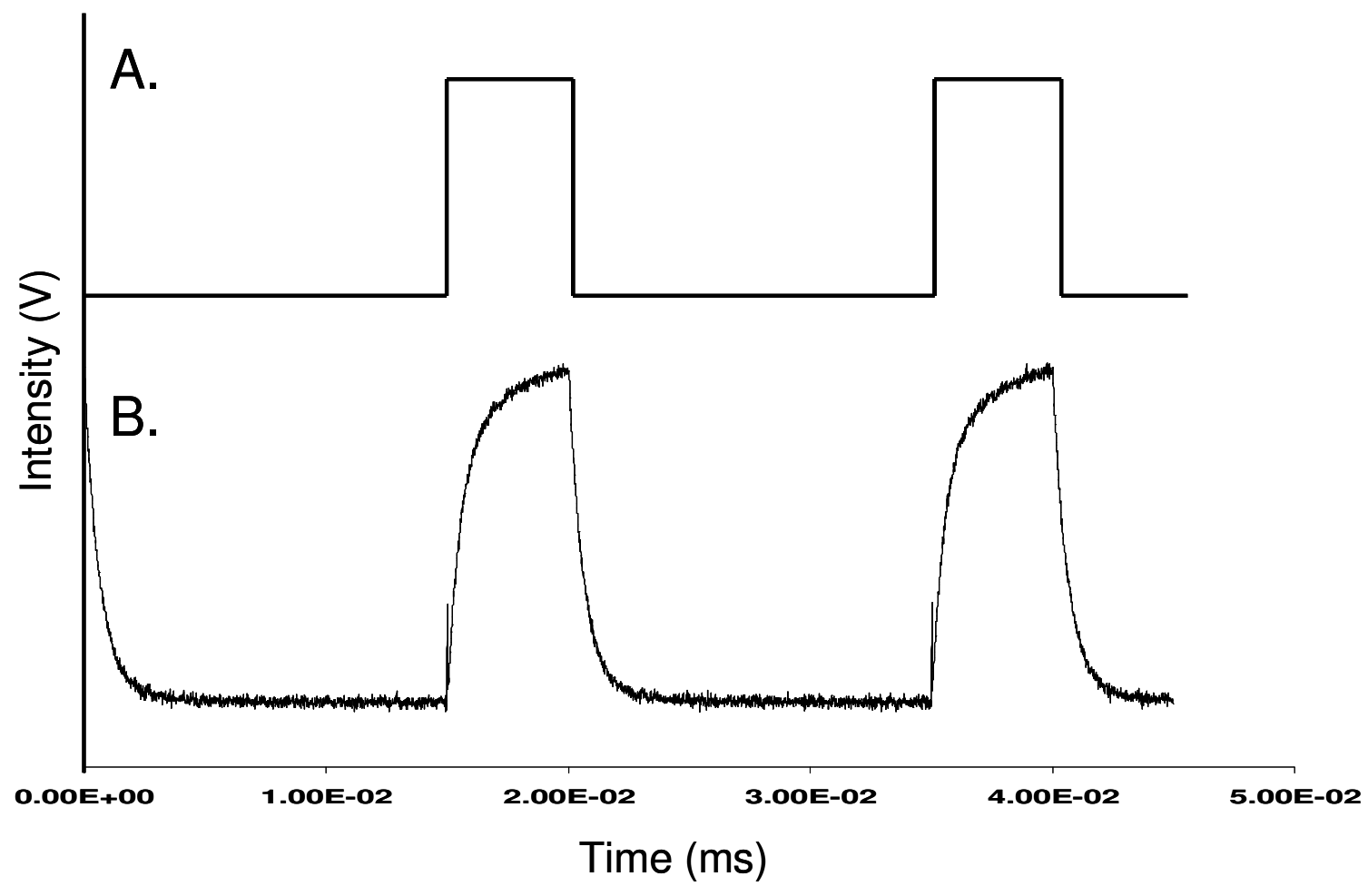

Figure 3.5. The temporal profiles of A) the applied power of the pulsed glow discharge; and B) the response voltage from the Langmuir probe (later converted to current during data processing). 

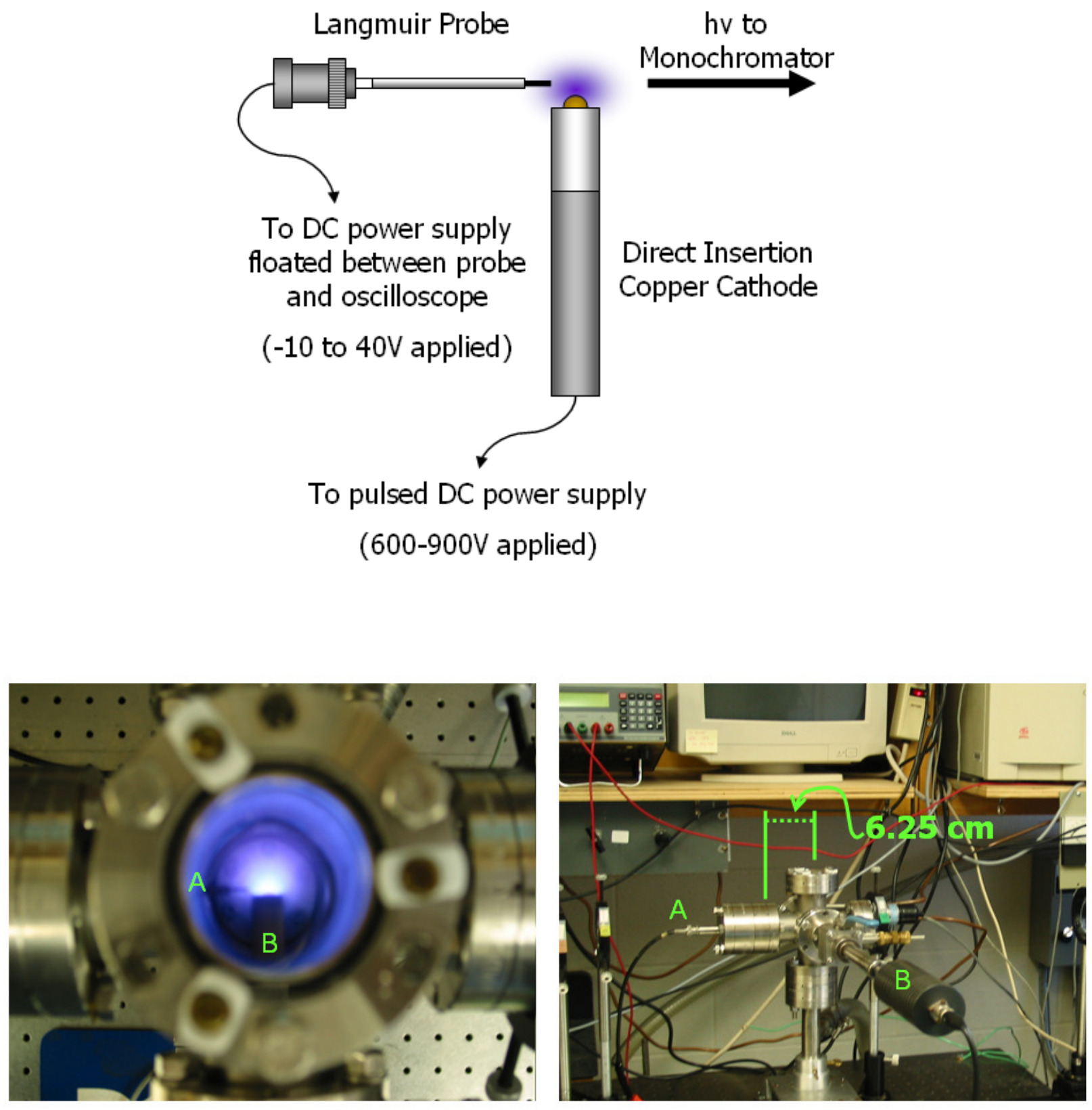

Figure 3.6. Close-up schematic of the Langmuir probe and cathode orientation, as well as pictures showing the top and side views of the GD chamber with the A) Langmuir probe and B) cathode. 


\subsection{Theoretical}

When a conducting material is inserted into a plasma, the rapidly moving electrons in the plasma collect on its surface, resulting in negative charge on the conductor within a few nanoseconds. If the conductor is electrically isolated, floating, its potential will be negative with respect to the surrounding plasma causing positive ions to accelerate toward it (electrons are then repelled from the floating conductor) [4]. Eventually, the conductor will reach an equilibrium potential, called the floating potential $\left(\mathrm{V}_{\mathrm{f}}\right)$, with the same number of electrons and positive ions per second reaching the surface. Chapman describes the area around such an object as follows: "It is as though a potential energy 'hill' develops in front of the substrate. However, it is a downhill journey for ions from the plasma to the substrate, but uphill for the electrons, so that only those electrons with enough initial kinetic energy make it to the 'top', i.e. the substrate" [19]. This region of disturbed potential is known as the sheath and its thickness is on the order of a few Debye lengths $\left(\lambda_{D}\right)$. In a plasma, the Debye length is the scale over which electrons screen out the electric field allowing for space-charge separation. The densities and temperatures of charged species in the sheath match the bulk plasma conditions at the sheath edge (a region called the "presheath") [20].

If an external bias is applied to the conductor, current is drawn from the plasma with either ions or electrons being collected depending on the polarity of the bias [9]. The number and energies of the charged species incident on the probe, as well as its surface area and applied potential, dictate the magnitude of the current drawn [13]. The Langmuir probe method ascertains fundamental 
plasma parameters by observing the current to the probe as a function of the difference between the applied probe potential $\left(V_{a p}\right)$ and the local plasma pontential $\left(V_{s}\right)$. When $V_{a p}<<V_{s}$ (i.e. more negative) only ion current reaches the probe and electrons are repelled. This area of the I-V characteristic is known as the ion saturation region. When $V_{a p}>V_{s}$ (i.e. more positive) only electron current reaches the probe and ions are repelled. This area is known as the electron saturation region. When $V_{a p} \leq V_{s}$, only electrons with enough kinetic energy to overcome the retardation potential, or the potential energy 'hill' as described by Chapman, are able to reach the probe. This area is known as the electron retardation region and can be used as an exclusive energy selector [6]. The ion saturation region is used to determine the plasma density (ion density, $n_{i}$ ) and the electron retardation region is used to determine the electron energy distribution (EEDF) and the electron temperature $\left(T_{e}\right)$. If the ion density is equal to the electron density $\left(n_{i}=n_{e}\right)$, then $n_{e}$ can also be determined. Figure 3.6 shows a representative I-V characteristic trace with each region labeled.

Although the use of Langmuir probes is relatively simple, the treatment of the data is considerably more complex and adjustments in operating parameters can alter the applicable theory and calculations used. To analyze my data by conventional Langmuir theory, several assumptions are made: 1) the distribution of the electron energies is Maxwellian in nature (average kinetic energy is an order of $k T), 2$ ) that the plasma is in local thermal equilibrium (LTE), 3) that the particle mean free path exceeds the Debye length (collisionless sheath), and 4) that the probe radius exceeds the Debye length (thin sheath) [16]. The calculated 
values for the mean free path and the Debye length were $.081 \mathrm{~mm}$ and $0.10 \mathrm{~mm}$ respectively and the probe radius was $0.45 \mathrm{~mm}$. While the mean free path does not exceed the Debye length, calculations using conventional Langmuir theory will give a good approximation of electron temperature and density within the plasma.

The local plasma potential $\left(V_{s}\right)$ is the potential difference of the plasma volume with respect to the anode, or in our case the chamber walls. $V_{s}$ can be determined from the intersection of the electron retardation and electron saturation regions of the I-V characteristic (shown in Figure 3.6) or from the maximum of the first derivative of the electron current to the probe [4]. If the natural log of the current $\left(\ln \left|I-I_{i s}\right|\right)$ is plotted versus voltage $\left(V \equiv V_{a p}-V_{s}\right)$, electron temperature $\left(T_{e}\right)$ can be calculated from the inverse of the slope in the electron retardation region of the curve. Once $T_{e}$ and $V_{s}$ are known, electron number density $\left(n_{e}\right)$ can be calculated from Equation 3.1,

$$
I_{v}=I_{i s}-n_{e} \cdot e \cdot A_{p}\left(\frac{k T_{e}}{2 \pi \cdot m_{e}}\right)^{\frac{1}{2}} \exp \left(\frac{e \cdot V_{s}}{k T_{e}}\right)
$$

(Equation 3.1)

where $I_{v}$ is the current at plasma potential, $I_{i s}$ is the ion saturation current, $e$ is the charge of an electron, $A_{p}$ is the exposed probe area, $k$ is the Boltzmann constant, $m_{e}$ is electron mass, and $V_{s}$ is the plasma potential. Equation 3.1 shows that there is an exponential increase in the electron current until the probe voltage is equal to the plasma potential $\left(V=V_{a p}-V_{s}=0\right)$. Beyond the local plasma potential $(V>$ 
$V_{s}$ ), the current is space charge limited and increases linearly with the probe voltage [11]. 


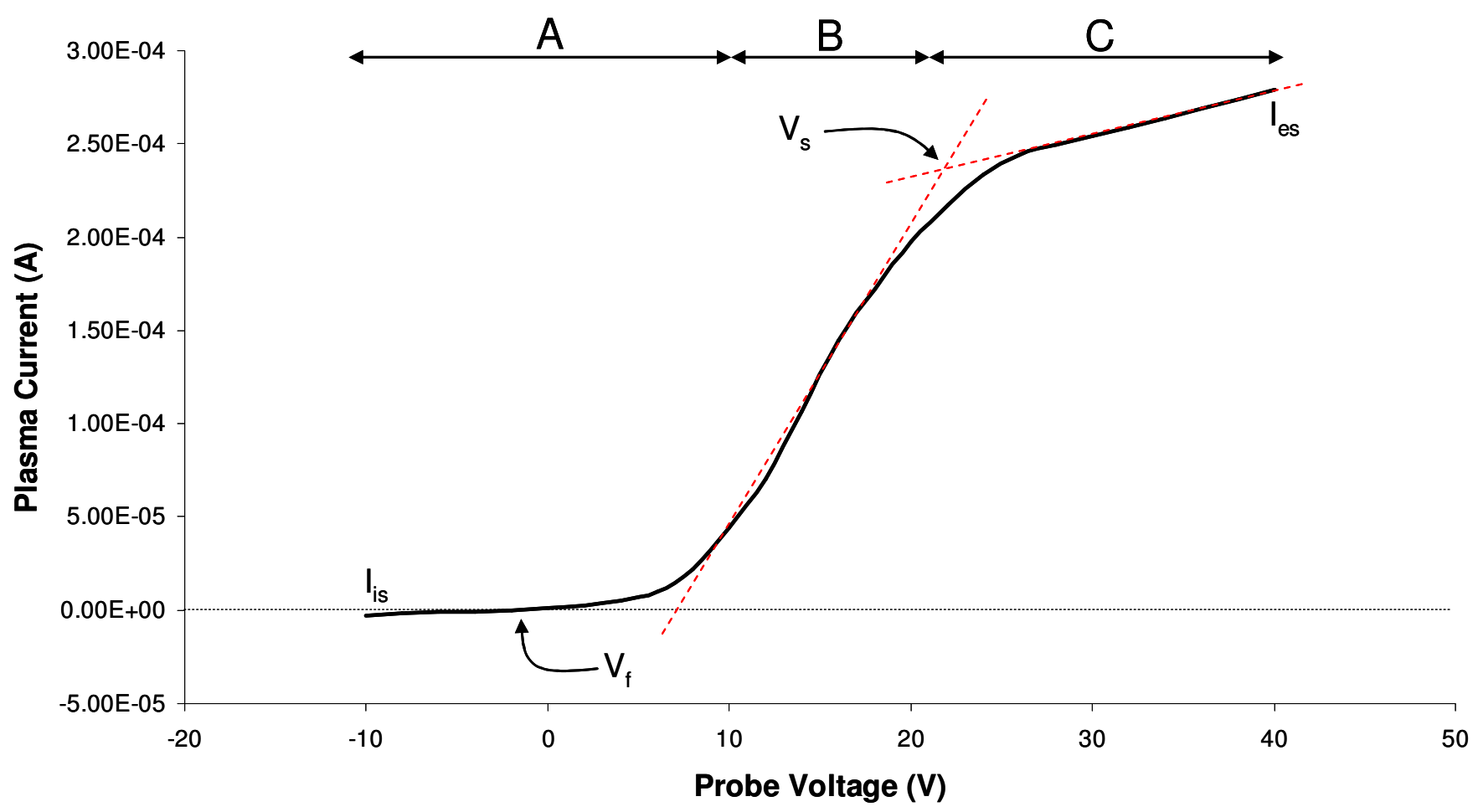

Figure 3.6. Schematic of a typical Langmuir I-V Characteristic trace with A) the ion saturation region (electrons repelled), B) the electron retardation region, and $\mathrm{C}$ ) the electron saturation region (ions repelled) and where $\mathrm{I}_{\text {is }}=$ ion saturation current, $l_{e s}=$ electron saturation current, $V_{f}=$ floating potential $(I=0)$, and $\mathrm{V}_{\mathrm{s}}=$ plasma potential [adapted from 11] 


\subsection{Results and Discussion}

\subsubsection{Temporal Langmuir Probe I-V Characteristics Electron Temperature $\left(T_{e}\right)$}

A glow discharge (GD) plasma is a hot, partially ionized gas (fractional ionization on the order of $10^{-4}$ ) consisting of roughly equal numbers of positive and negative charges, mostly electrons and positive ions. In plasma chemistry it is convenient to talk about the temperature of the charged species, but the speed distribution is a more precise description. For argon gas, the electrons are over 100,000 times less massive than their positive ion counterparts. In the presence of an electric field, the lighter mass of the electrons ensures them greater mobility and a faster mean speed. Moreover, because of the mass inequality, an electron will lose only a small fraction of its energy in an elastic collision with a neutral or ion which is in stark contrast to ionic collisions [21]. Thus, the word "hot" in the definition refers mostly to the electrons which have an average velocity some 3,000 times greater than that of the neutral molecules or positive ions [4]. In a typical GD plasma, the electrons are 25,000 - 50,000 K while the neutrals and ions have more modest temperatures ( 500 K).

Figures 3.7 and 3.8 show a characteristic I-V trace and $\mathrm{T}_{\mathrm{e}}$ profile for both the plateau and afterpeak temporal regimes. The slope of $\ln (I)$ vs. $V$ yielded straight lines for all plots, indicating that the distribution was Maxwellian. Calculated values for $T_{e}$ ranged between 3.3 and $4.7 \mathrm{eV}$ for the plateau region and 3.8 and $6.0 \mathrm{eV}$ for the afterpeak region with average values for both equal to $3.6 \mathrm{eV}(\sim 41,000 \mathrm{~K})$ and $4.4 \mathrm{eV}(\sim 50,000 \mathrm{~K})$ respectively. In all cases, the 
measured $T_{e}$ for the afterpeak was higher than that of the plateau with the average difference between the two temperatures equal to $0.8 \mathrm{eV}$. The calculated RSD was $45 \%$ for both the plateau and afterpeak making it difficult to assert whether any temperature difference between the two regions truly exists. Furthermore, the net drift of electrons with respect to the probe tends to overestimate the electron temperature, giving a value some 3 times greater than predicted [22].

While the range of $T_{e}$ values are in good agreement with those of other GD-Langmuir investigations $[6,16,17]$, the spike in $T_{e}$ values in the afterglow contradicts conventional descriptions of the pulsed GD afterpeak which suggest that upon pulse termination, the electron population will quickly thermalize to the gas temperature as electrons lose energy via collisions in the plasma [23].

However, similar results were observed by Overzet and Kleber in their Langmuirprobe investigations into the electron properties of pulsed gaseous electronics conference reference cell (GEC) discharges in pure Ar [24]. They attributed this increase to reactions between metastable argon atoms that yield energetic electrons as follows:

$$
\mathrm{Ar}^{\mathrm{m}}+\mathrm{Ar}^{\mathrm{m}} \rightarrow \mathrm{Ar}^{+}+\mathrm{Ar}^{0}+\mathrm{e}^{-} \text {(fast) } \quad \mathrm{EEDF}=7.3-7.9 \mathrm{eV} \quad \text { (Eqn. 3.2) }
$$

In their experiments, electron energy distribution function (EEDF) peaks of $\sim 7.5$ $\mathrm{eV}$ during the afterglow confirmed the presence of these high energy electrons [24]. Likewise, Wenig et. al. observed high constant values for the mean electron energy late in the afterglow of a pulsed low pressure inductively coupled plasma. The group undertook modeling experiments to better understand the phenomena 
which demonstrated that energy losses of the electrons could be compensated by re-heating via superelastic collisions from the upper to the lower metastable level, and by chemo-ionization involving the metastable levels $[25,26]$.

\section{Electron Number Density $\left(\mathrm{n}_{\mathrm{e}}\right)$}

As stated in Section 3.3, the electron number density $\left(n_{e}\right)$ can be calculated from Equation 3.1 once the electron temperature $\left(T_{e}\right)$ and the local plasma potential $\left(V_{s}\right)$ have been determined. In most cases the first derivative method was used to find $V_{s}$ unless no discernable maxima could be found, in which case, the intersection method was used. For applicable runs, $V_{s}$ values were determined using both techniques. There was good agreement between the methods with differences in a single run of less than $\pm 1 \mathrm{~V}$. Overall, $\mathrm{V}_{\mathrm{s}}$ values ranged from $3.4-13.3 \mathrm{~V}$ with an average of $6.63 \mathrm{~V}$ and no statistical difference between the average values for the plateau and afterpeak was found.

Calculated values for $n_{e}$ ranged between $9.1 \times 10^{9}$ and $3.3 \times 10^{10} \mathrm{~cm}^{-3}$ for the plateau region and $4.3 \times 10^{9}$ and $1.5 \times 10^{10} \mathrm{~cm}^{-3}$ for the afterpeak region. On average, the afterpeak $n_{e}$ dropped to about $47 \%$ of the plateau value. While these densities fall well within the range of other GD-Langmuir studies, the drop in $n_{e}$ during the afterpeak when compared with the plateau goes against traditional pulsed GD thinking as discussed in Chapter 2. Popular theory proposes that electron-ion recombination followed by radiative decay is the most likely cause of afterpeak emissions. However, in order to account for the emission intensification found in the after peak by this mechanism, a rise in 
electron density of about two orders of magnitude would be required [23]. While this increase has never been found experimentally, similar drops in $n_{e}$ have been found for ms-pulsed GD investigated by Thompson laser-scattering [27], pulsed GEC discharges using Langmuir probes [24], and through Monte Carlo models of ms-pulsed GD plasmas [26]. In the latter example, the calculated electron density dropped to within $38 \%$ of the plateau value.

Recent modeling experiments by Bogaerts strongly suggest that dissociative recombination between $\mathrm{Ar}_{2}{ }^{+}$ions and electrons, rather than electronion recombination, is the most likely cause of the afterpeak emission intensification [26]. Production of molecular argon ions $\left(\mathrm{Ar}_{2}^{+}\right)$in the GD plasma occurs by several processes:

$\underline{\mathrm{Ar}^{+} \text {to } \mathrm{Ar}_{2}}{ }^{+}$ion conversion

$$
\mathrm{Ar}^{+}+2 \mathrm{Ar}^{0} \rightarrow \mathrm{Ar}_{2}{ }^{+}+\mathrm{Ar}^{0} \quad \mathrm{k}=2.7 \times 10^{-31} \mathrm{~cm}^{6} \mathrm{~s}^{-1}
$$

$\underline{\text { Ar metastable-metastable associative ionization }}$

$$
\mathrm{Ar}_{\mathrm{m}}{ }^{*}+\mathrm{Ar}_{\mathrm{m}}{ }^{*} \rightarrow \mathrm{Ar}_{2}{ }^{+}+\mathrm{e}^{-} \quad \mathrm{k}=5.7 \times 10^{-10} \mathrm{~cm}^{3} \mathrm{~s}^{-1}
$$

$\underline{\text { Hornbeck Molnar associative ionization }}$

$$
\mathrm{Ar}^{\star \star}+\mathrm{Ar}^{0} \rightarrow \mathrm{Ar}_{2}^{+}+\mathrm{e}^{-} \quad \mathrm{k}=2 \times 10^{-9} \mathrm{~cm}^{3} \mathrm{~s}^{-1}
$$

Of these, Hornbeck Molnar associative ionization is thought to be the most important contributor of molecular argon ions during the afterpeak regime. This is due to the enhanced population of highly excited argon $\left(A r^{* *}\right)$ early-on in the afterglow that causes the ionization rate to spike over $1.0 \times 10^{-17} \mathrm{~s}^{-1}$ upon pulse termination [26]. This process is offset by dissociative recombination between molecular argon ions and electrons in the following reaction: 


\section{Electron-ion dissociative recombination}

$$
\mathrm{Ar}_{2}{ }^{+}+\mathrm{e}^{-} \rightarrow \mathrm{Ar}^{\star *}+\mathrm{Ar}^{0} \quad \mathrm{k}=8.5 \times 10^{-7} \mathrm{~cm}^{3} \mathrm{~s}^{-1}
$$

Some of Bogaerts modeling results are displayed in Figure 3.9. Figure 3.9.1 shows the calculated population densities of Ar atoms in various excited levels as a function of time with $\mathrm{Ar}_{2}{ }^{+}$included in the modeling, while Figure 3.9.2 shows the same profiles with $\mathrm{Ar}_{2}{ }^{+}$excluded from the modeling. A clear discrepancy can been seen in the afterpeak populations for levels with excitation energies of 15.2 $\mathrm{eV}$ or higher $(\mathrm{d}-\mathrm{h})$. Although a small afterpeak is evident in 3.9.2h, demonstrating that ion-electron recombination plays at least a minor role in the formation of elevated ion intensities in the afterglow, the populations are still an order of magnitude lower than for dissociative recombination. Figure 3.9.3 shows the calculated rates of a) dissociative recombination and b) ion-electron recombination as a function of time [26]. 
Table 3.1 lists the average operating power, $n_{e}$ and $T_{e}$ with the percent relative standard deviations (RSD) for both, the ion saturation current ( $\left.l_{\text {is }}\right)$, electron saturation current $\left(\mathrm{l}_{\mathrm{es}}\right), \mathrm{V}_{\mathrm{s}}$, and the floating potential $\left(\mathrm{V}_{\mathrm{f}}\right)$ for both the plateau and afterpeak temporal regions.

\section{Table 3.1}

\begin{tabular}{|c|c|c|c|c|c|c|c|c|c|}
\hline $\begin{array}{c}\text { Temporal } \\
\text { Region }\end{array}$ & $\begin{array}{c}\text { Power } \\
\text { (W) }\end{array}$ & $\begin{array}{c}\mathrm{n}_{\mathrm{e}} \\
\left(\mathrm{cm}^{-3}\right)\end{array}$ & $\begin{array}{c}\text { RSD } \\
(\%)\end{array}$ & $\begin{array}{c}\mathrm{T}_{\mathrm{e}} \\
(\mathrm{eV})\end{array}$ & $\begin{array}{c}\text { RSD } \\
(\%)\end{array}$ & $\begin{array}{l}I_{\text {is }} \\
(V)\end{array}$ & $\begin{array}{l}I_{\text {es }} \\
(V)\end{array}$ & $\begin{array}{l}V_{s} \\
(V)\end{array}$ & $\begin{array}{l}V_{f} \\
(V)\end{array}$ \\
\hline Plateau & .668 & 1.6E10 & 17. & 3.6 & 45. & $-3.28 E-06$ & 3.16E-04 & 6.68 & -3.60 \\
\hline Afterpeak & .668 & 7.6E09 & 19. & 4.4 & 44. & $-2.08 E-06$ & $1.58 \mathrm{E}-04$ & 6.59 & -4.36 \\
\hline
\end{tabular}




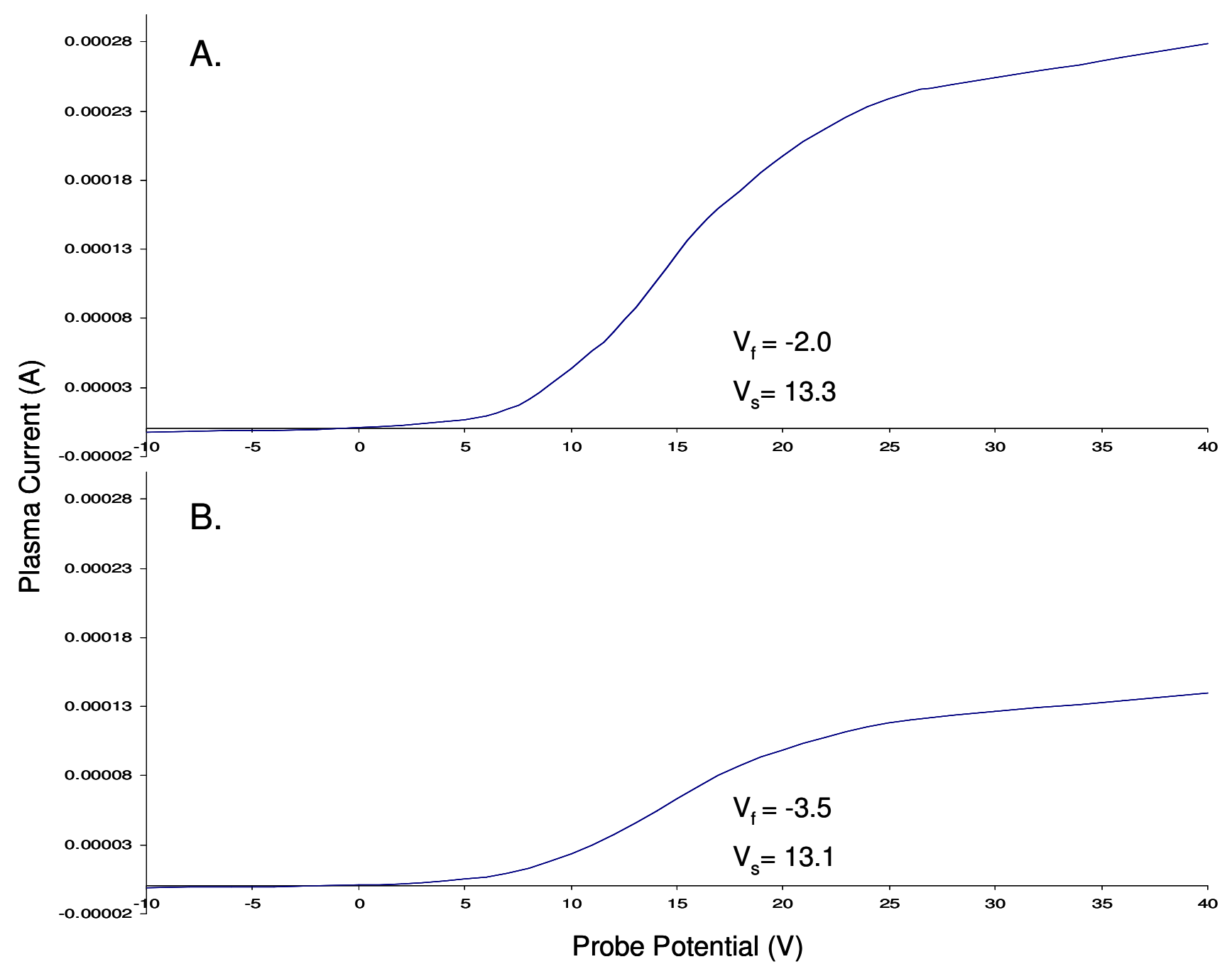

Figure 3.7. I-V characteristic traces during the A) plateau (4.750 $5.000 \mathrm{~ms})$ and $B)$ afterpeak $(5.001-5.101 \mathrm{~ms})$. Floating potentials $\left(\mathrm{V}_{\mathrm{f}}\right)$ and plasma potentials $\left(V_{s}\right)$ for the plateau and afterpeak are listed on each plot. Discharge pressure 0.75 torr, operating power $.64 \mathrm{~W}$, and sampling distance $7 \mathrm{~mm}$. 


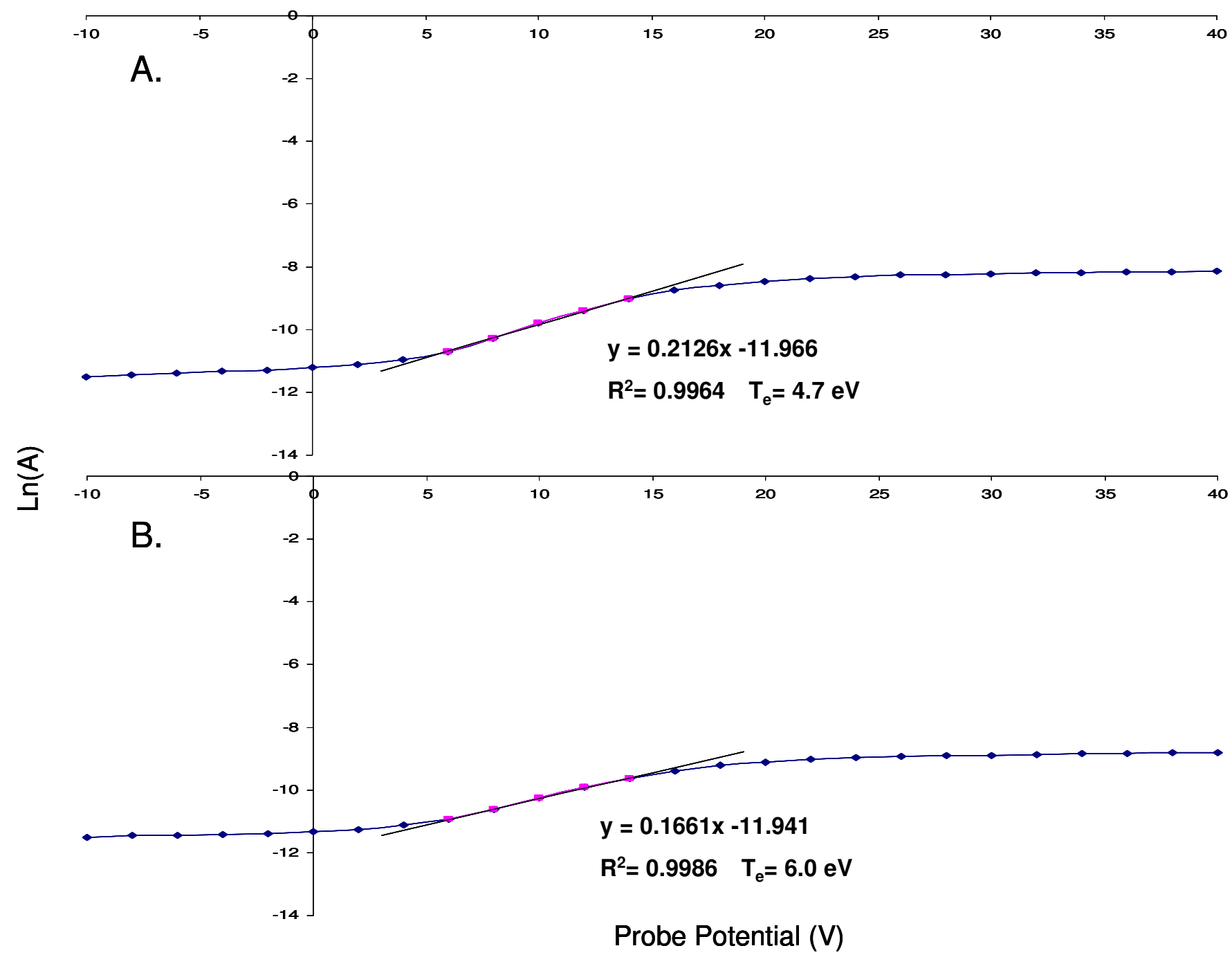

Figure 3.8. Plot of the natural log of the current $\left(\ln \left|I-I_{\text {is }}\right|\right)$ versus the probe potential $\left(V_{a p}-V_{s}\right)$ for the A) plateau $(4.750-5.000 \mathrm{~ms})$ and B) afterpeak (5.001 $-5.101 \mathrm{~ms})$. The electron temperature $\left(T_{e}\right)$ was determined by the inverse of the slope in the electron retardation region of the plot (shown in pink). Discharge pressure 0.75 torr, operating power $.64 \mathrm{~W}$, and sampling distance $7 \mathrm{~mm}$. 


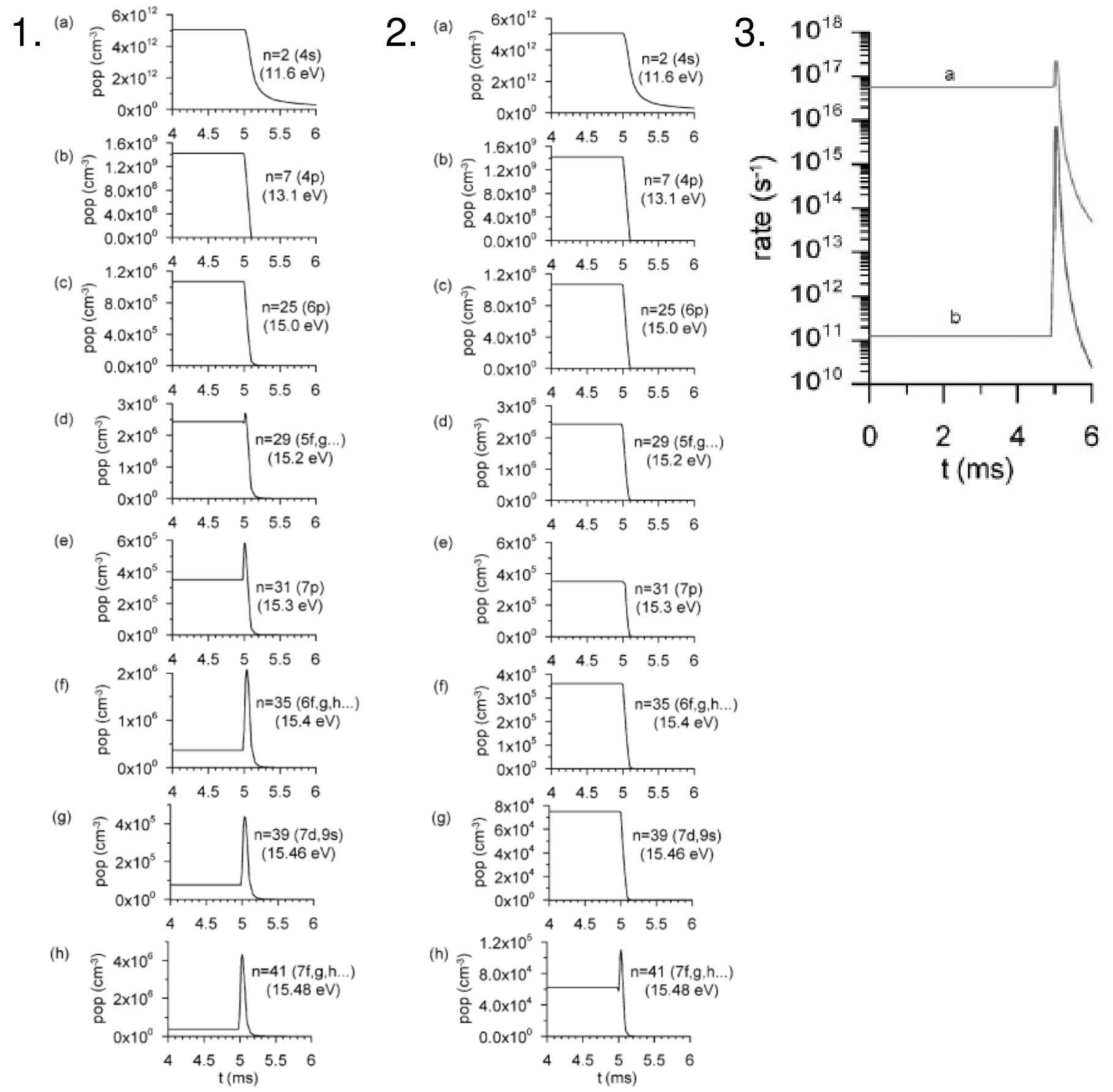

Figure 3.9. Calculated level populations of several Ar excited levels as a function of time with $1 \mathrm{a}-\mathrm{h}) \mathrm{Ar}_{2}{ }^{+}$ions included and 2a-h) $\mathrm{Ar}_{2}{ }^{+}$ ions excluded from the calculations. 3) Calculated rates of a) dissociative recombination and b) ion-electron recombination as a function of time [26]. 


\subsubsection{Spatial Characteristics}

Axial profiles were measured by varying the position of the direct insertion probe (copper cathode) from 5-23 $\mathrm{mm}$ with respect to the Langmuir probe. Vertical profiles were also measured using four Langmuir probes of differing lengths. Probe 1 marked the center of the plasma at the zero position $(5.4 \mathrm{~mm}$ in total length), while each consecutive probe, numbers 2, 3 and 4 , measured 1 $\mathrm{mm}$ in from the center $(5.3,5.2$, and $5.1 \mathrm{~mm}$ in total length or $1-3 \mathrm{~mm}$ from the center of the plasma respectively). A schematic representation of the axial and vertical distances analyzed is shown in Figure 3.10. Figure 3.11 shows the axial profiles for electron density and electron temperature, whereas Figure 3.12 shows the average electron density and electron temperature for each vertical distance, $0-3 \mathrm{~mm}$. The average was taken for at least three runs on each probe at a distance of $5 \mathrm{~mm}$ and with an operating power and discharge pressure of .64 W and .75 torr respectively.

Both Figures 3.11.A and 3.12.A show little differences among $n_{e}$ values along either the axial or vertical axes. Average values for $n_{e}$ were $5.4 \times 10^{9}, 6.0 \times$ $10^{9}, 4.0 \times 10^{9}$, and $1.0 \times 10^{10} \mathrm{~cm}^{-3}$ for each Langmuir probe measuring $0-3 \mathrm{~mm}$ respectively, with the average standard deviation equal to $\pm 3.2 \times 10^{9} \mathrm{~cm}^{-3}$. These data agree with spatially resolved measurements made throughout the bulk of the negative glow region by other investigators $[6,16]$, however, Marcus did see a small decrease in $n_{e}$ near the far end of the plasma. Because of the low pressures used and high diffusion rates found within glow discharge plasmas, the lack of dependence of the electron density on the cathode distance is expected [16]. 
Figures 3.11.B and 3.12.B show the axial and vertical profiles of electron temperature. $T_{e}$ showed little statistical change when measured axially; however, a slight decrease was seen as the average distance from the cathode was measured vertically. Although minor, this decrease is not surprising because electron heating should take place close to the copper cathode surface near the edge of the cathode sheath. Average values for $\mathrm{T}_{\mathrm{e}}$ were 4.2, 3.7, 3.4, and $3.3 \mathrm{eV}$ for probes $1-4$ respectively, with the average standard deviation equal to \pm 0.5 $\mathrm{eV}$.

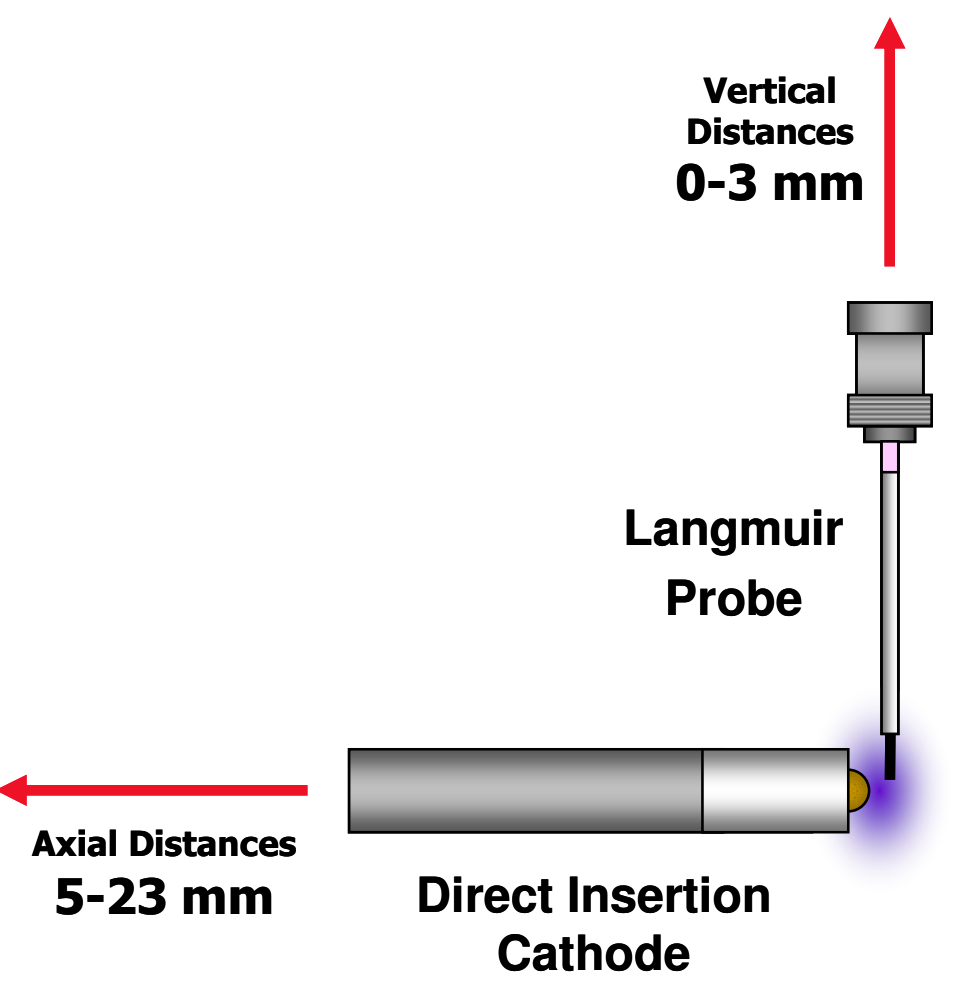

Figure 3.10. Schematic representation of the axial and vertical distances analyzed for the spatially resolved profiles. 


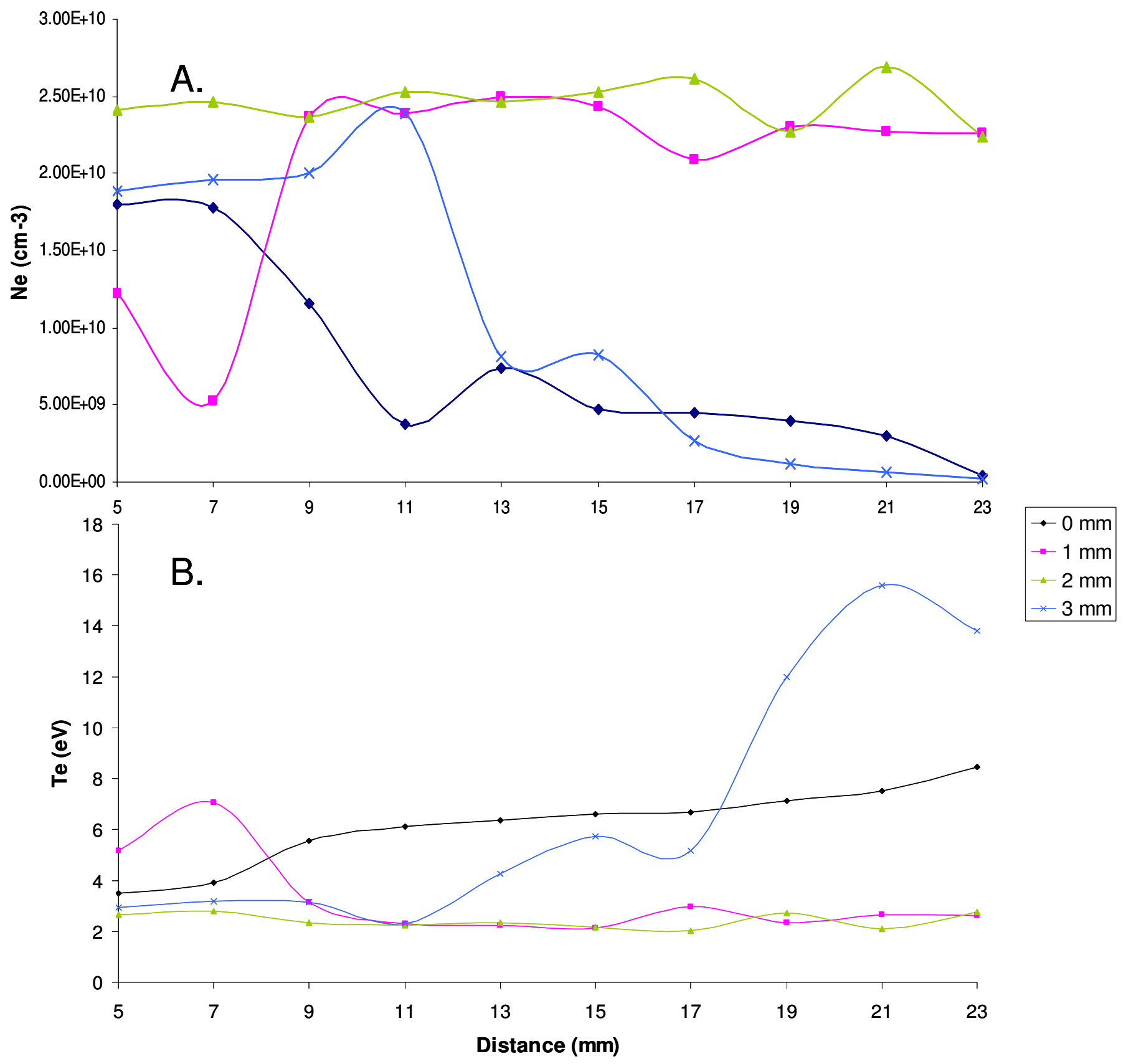

Figure 3.11. Axial profiles of $A$ ) electron density and $B$ ) electron temperature using probes of differing vertical distance $(0-3 \mathrm{~mm}$ from the center of the plasma respectively). Sampled during the plateau regime $(4.75-5.00 \mathrm{~ms})$ with discharge pressure 0.75 torr and operating power $.60 \mathrm{~W}$. 

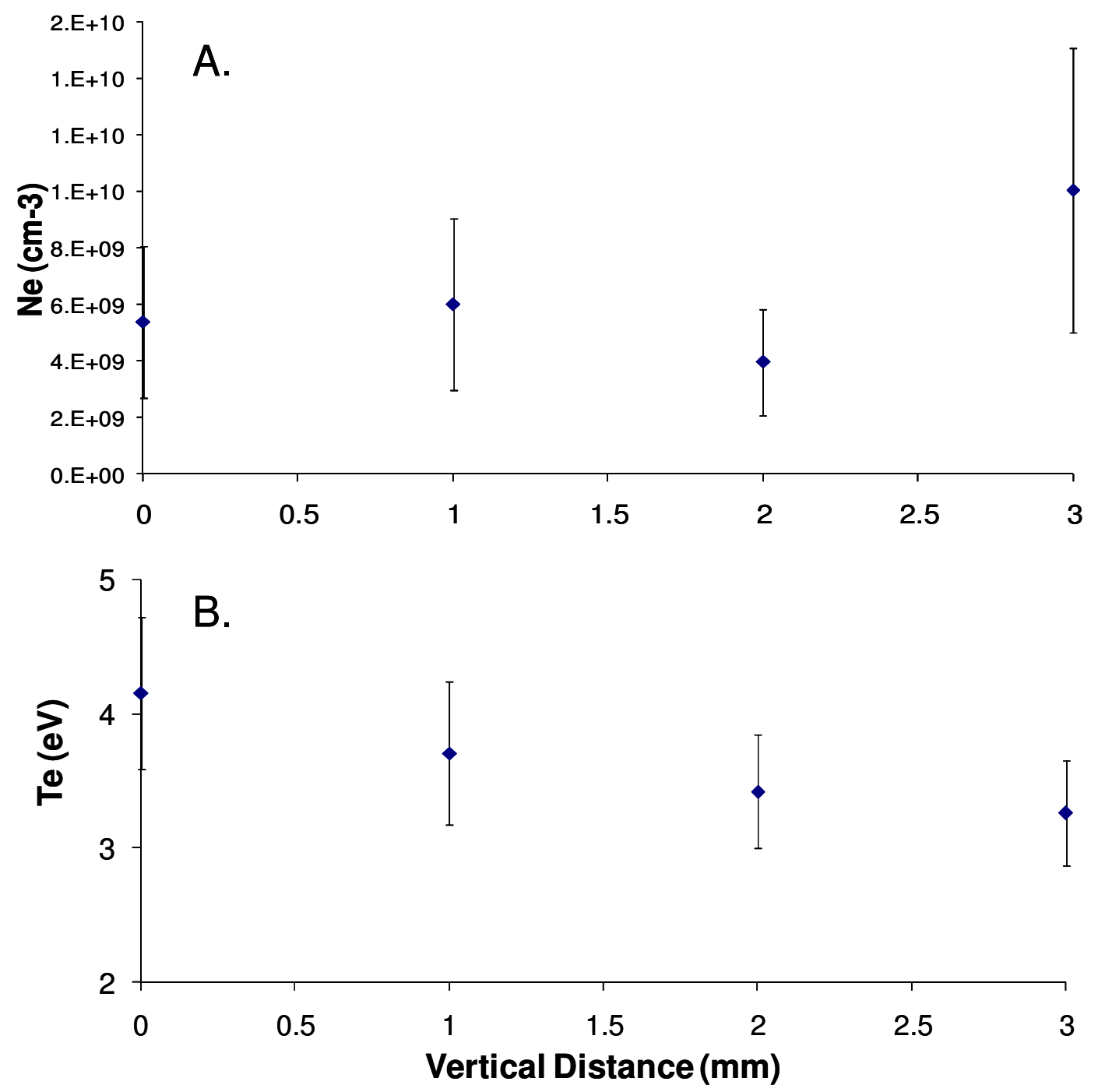

Figure 3.12. Average profiles of A) electron density and B) electron temperature versus vertical distance from the cathode $(0-3 \mathrm{~mm}$ from the center of the plasma respectively) with the direct insertion probe stationary. Sampled during the plateau regime $(4.75-5.00 \mathrm{~ms})$. Discharge pressure 0.75 torr and operating power $.64 \mathrm{~W}$. 


\subsubsection{Spectral Characteristics}

To ascertain any effects the Langmuir probe might have on the plasma, simultaneous spectral data of several copper lines were collected using a monochromator. As discussed in Chapter 2, the copper emission afterpeaks (in this case $\mathrm{Cu} \mathrm{I,} 368.7 \mathrm{~nm}$ ) reach a maximum approximately $0.2 \mathrm{~ms}$ after pulse termination ( 5.2 ms). This rise in the afterpeak emission of Copper I lines, including 324.7 and $368.7 \mathrm{~nm}$, has been determined by optical emission, atomic absorption, and mass spectrometric methods and is well documented [28-31]. The rise in the copper ion population is closely associated with argon metastable atoms and Penning ionization during the afterpeak regime.

Figure 3.13 shows the $A$ ) raw and $B$ ) normalized temporal profiles of optical emission data for $\mathrm{Cu}(\mathrm{I}) 324.7$ and $368.7 \mathrm{~nm}$. The pink line is optical data taken with no voltage applied to the Langmuir probe whereas the blue line is optical data take while 65 volts was applied to the Langmuir probe. The raw data in $3.13 \mathrm{~A}$ shows that the use of the Langmuir probe increases the relative intensity for the measured transition. However, there is little difference in the normalized profiles shown in 3.13B demonstrating that the use of the Langmuir probe did not affect the overall temporal emissions and therefore the sputtering and ionization efficiencies within a particular time regime. Future experiments are planned to examine the affects of lower voltages applied to the probe, between 0 and $40 \mathrm{~V}$. 


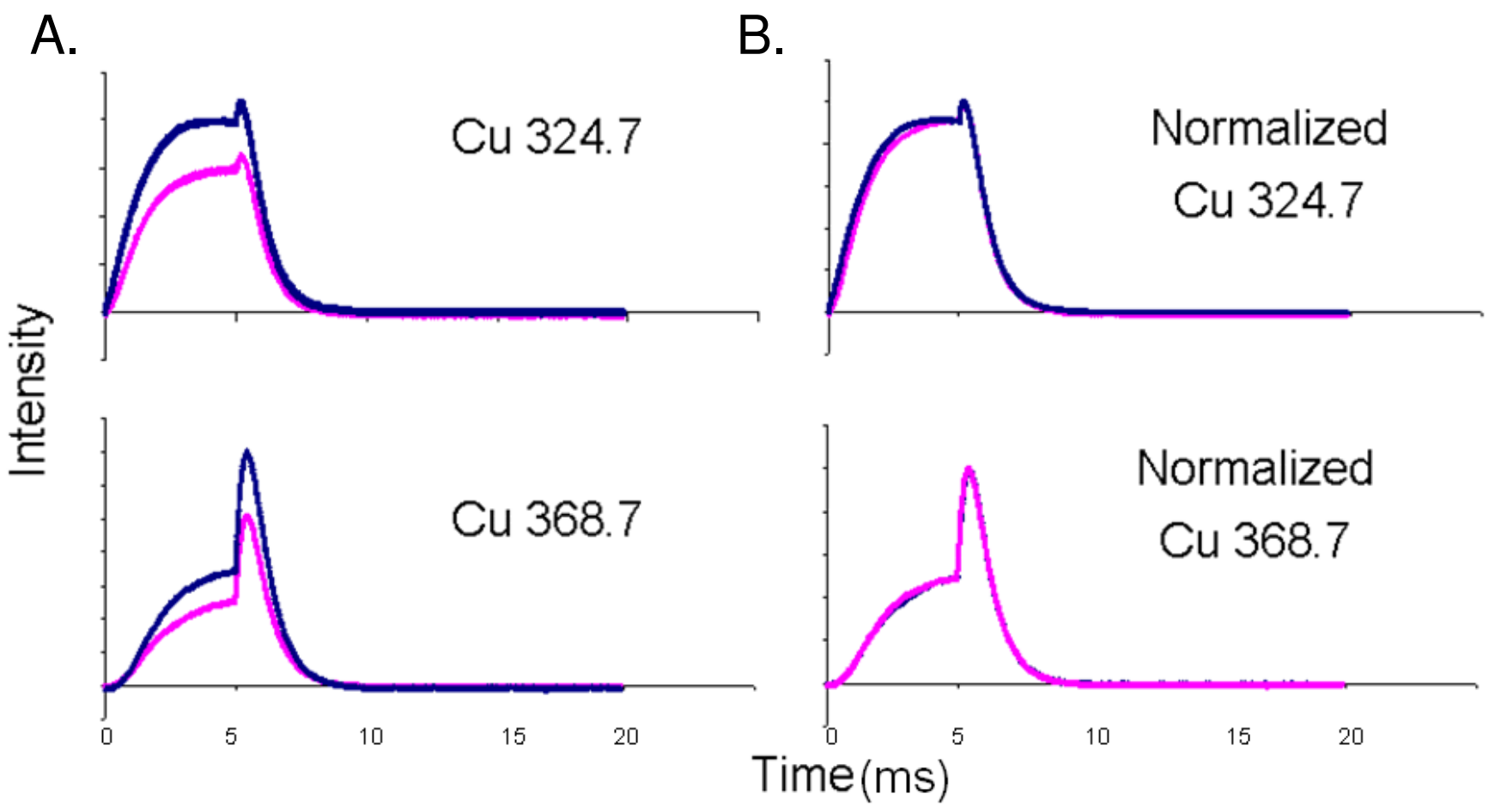

Figure 3.13. Temporal profiles of $A$ ) raw and $B$ ) normalized optical emission data for $\mathrm{Cu}(\mathrm{I}) 324.7$ and $368.7 \mathrm{~nm}$ with no voltage (pink) and $65 \mathrm{~V}$ (blue) applied to the Langmuir probe. Discharge pressure 0.75 torr and sampling distance $5 \mathrm{~mm}$. 


\subsection{Conclusions}

Time-resolved Langmuir probe studies were undertaken to elucidate the excitation and ionization processes in a direct current pulsed glow discharge plasma. Electron densities and temperatures were derived from the Langmuir probe results and attention was paid to differences observed between the poweron, plateau, and power-off, afterpeak, regimes. Calculated values for electron density $\left(n_{e}\right)$ ranged between $9.1 \times 10^{9}$ and $3.3 \times 10^{10} \mathrm{~cm}^{-3}$ for the plateau region and $4.3 \times 10^{9}$ and $1.5 \times 10^{10} \mathrm{~cm}^{-3}$ for the afterpeak region. On average, the afterpeak $n_{e}$ dropped to about $47 \%$ of the plateau value. Values for electron temperature $\left(T_{e}\right)$ ranged between 3.3 and $4.7 \mathrm{eV}$ for the plateau region and 3.8 and $6.0 \mathrm{eV}$ for the afterpeak region with average values for both equal to $3.6 \mathrm{eV}$ $(\sim 41,000 \mathrm{~K})$ and $4.4 \mathrm{eV}(\sim 50,000 \mathrm{~K})$ respectively. The calculated RSD was $45 \%$ for both the plateau and afterpeak making it difficult to assert whether any temperature difference between the two regions truly exists.

In the multipulse studies discussed in Chapter 2, it was proposed that the following processes take place in sequence after the power termination.

1) electron thermalization;

2) argon ion-electron recombination-decay forming argon metastable atoms;

3) Penning ionization of copper atoms yielding copper ions;

4) copper ion-electron recombination-decay forming excited copper atoms. While argon ion-electron recombination-decay plays a non-negligible role in the formation of the afterpeak, it cannot account for the majority of afterglow 
emissions because it requires a rise in electron density of about two orders of magnitude not evident in our data. Rather, it is suggested here that dissociative recombination between molecular argon ions $\left(\mathrm{Ar}_{2}^{+}\right)$and electrons is the principal process responsible for afterpeak emissions.

Upon power termination, both electron ionization and charge transfer processes halt and the ions formed by these processes quickly decay to a baseline value. The energetic metastable atoms (11.55 and $11.72 \mathrm{eV}$ ) play a central role in ionization and excitation during the afterpeak regime as Penning ionization becomes dominant. At the same time, the population of highly excited argon levels increases. This is due to electron-ion dissociative recombination, but also to a lesser degree from ion-electron recombination.

The high energy ionization processes dominant within the afterglow may also account for the increase in electron temperature found within the afterpeak regime. Electrons are thought to thermalize upon pulse termination due to the drop in potential at the cathode. It is possible that re-heating of the electrons takes place via superelastic collisions from the upper to the lower metastable levels or by chemo-ionization involving the metastable levels. This, along with the decrease in the electron population could cause the average energy of the remaining electrons to increase.

Spatially-resolved Langmuir probe studies allowed for the measurement of electron densities and temperatures within the plasma as a function of axial and vertical distance from a direct insertion copper cathode while simultaneous spectral data were collected to determine any affects the Langmuir probe might 
have on the plasma. Although the raw data showed an increase in the relative intensity for a measured transition, there was little difference in the normalized profiles. This demonstrated that the use of the Langmuir probe did not affect the overall temporal emission profiles and therefore the sputtering and ionization efficiencies within a particular time regime over another. 


\subsection{References}

1. G. L. Rogoff, Ed., IEEE Transactions on Plasma Science, 19(1991) 989.

2. I. Langmuir, Oscillations In lonized Gases, Proc. Nat. Acad. Sci. U.S., 14(1928) 627-637.

3. I. H. Hutchinson, Principles of Plasma Diagnostics $2^{\text {nd }}$ Ed., Cambridge University Press: Cambridge, UK (2002).

4. D. N. Ruzic, Electric Probes for Low Temperature Plasmas, American Vacuum Society Education Committee: New York, NY (1994).

5. J. S. Crighton, J. Carroll, B. Fairman, J. Haines, M. Hinds, Atomic Spectrometry Update-Industrial Analysis: metals, chemicals, and advanced materials, J. Anal. Atomic Spect., 11 (1996) 461R-508R.

6. D. Fang, R. K. Marcus, Use of cylindrical Langmuir probe for the characterization of charged particle populations in a planar, diode glow discharge device, Spectrochimica Acta B, 45 (1990) 1053-1074.

7. W. W. Harrison, C. Barshick, J. A. Kingler, P. H. Ratcliff, Y. Mei, Glow discharge techniques in analytical chemistry, Anal. Chem., 62 (1990) 943A949A.

8. C. L. Lewis, M. A. Moser, D. E. Dale, W. Hang, C. Hassell, F. L. King, V. Majidi, Time-gated pulsed glow discharge: Real-time chemical speciation at the elemental, structural, and molecular level for gas chromatography timeof-flight mass spectrometry. Analytical Chemistry 75 (2003) 1983-1996.

9. A. Keesee, Neutral Density Profiles in Argon Helicon Plasmas. West Virginia University Libraries: Morgantown, WV (2006).

10. Y. Yuancai, R. K. Marcus, Langmuir probe study of the charged particle characteristics in an analytical radio frequency-glow discharge. Roles of discharge conditions and sample conductivity. Spectrochimica Acta B, 51 (1996) 509-531.

11. F. F. Chen, R. H. Huddlestone, Plasma Diagnostic Techniques, Academic Press: New York, NY (1964).

12. D. L. Rudakov, M. G. Shats, R. W. Boswell, C. Charles, J. Howard, Overview of probe diagnostics on the H-1 heliac, Rev. Sci. Inst. 70 (1999) 476-479.

13. R. K. Marcus, Glow Discharge Spectroscopies, Plenum Press: New York (1993).

14. D. Fang, R. R. Williams, R. K. Marcus, Design and evaluation of a computer controlled langmuir probe system for glow discharge plasma diagnostics, J. Anal. Atomic Spect. 5 (1990) 569-573.

15. A. P. Paranjpe, J. P. McVittie, S. A. Self, A tuned Langmuir probe for measurements in rf glow discharges, J. Appl. Phys., 67 (1990) 6718-6727.

16. M. J. Heintz, G. M. Hieftje, Langmuir-probe measurements of a pulsed and steady-state if glow-discharge source and of an if planar-magnetron source, Spectrochimica Acta B, 51 (1996) 1629-1646. 
17. W. H. Tao, H. K. Yasuda, Measurement of spatial distributions of electron density and electron temperature in direct current glow discharge by double langmuir probes, Plasma Chem. And Plasma Proc., 22 (2000) 297-311.

18. Y. Fei, IV Ramp Circuits for Langmuir Probes, http://metalab.uniten.edu.my/ chandan/ August 30, 2007.

19. B. N. Chapman, Glow Discharge Processes, John Wiley and Sons: New York (1980).

20. J. D. Swift, Electrical Probes for Plasma Diagnostics, lliffe Books Ltd.: London, UK (1970).

21. A. M. Howatson, An Introduction to Gas Discharges, Pergamon Press: Oxford, UK (1965).

22. T.E. Sheridan, J. Goree, Langmuir-probe characteristics in the presence of drifting electrons. Physical Review E, 50 (1994) 2991-2996.

23. A. Bogaerts, R. Gijbels, G. P. Jackson, Modeling a millisecond pulsed glow discharge: Investigation of the afterpeak, J. Anal. Atomic Spect., 18 (2003) 533-548.

24. L. J. Overzet, J. Kleber, Effect of metastable atom reactions on the electron energy probability functions in afterglows, Plasma Sources Sci. Technol., 7 (1998) 512-523.

25. G. Wenig, M. Schulze, P. Awakowicz, A. V. Koedell, Modeling of pulsed low-pressure plasmas and electron re-heating in the late afterglow. Plasma Sources Sci. Technol., 15 (2006) S35.

26. A. Bogaerts, The afterglow mystery of pulsed glow discharges and the role of dissociative electron-ion recombination, J. Anal. Atomic Spect. 22 (2007) 502-512.

27. G. Gamez, A. Bogaerts, G. M. Hieftje, Temporal and spatially resolved laser-scattering plasma diagnostics for the characterization of a ms-pulsed glow discharge, J. Anal. Atomic Spect. 21 (2006) 350-359.

28. F. L. King, C. K. Pan, Temporal signal profiles of analytical species in modulated glow discharge plasmas, Anal. Chem. 65 (1993) 735-739.

29. C. Lewis, S. K. Doorn, D. M. Wayne, F. L. King, V. Majidi, Characterization of a pulsed glow discharge laser ablation system using optical emission, Appl. Spect., 54 (2000) 1236-1244.

30. G. P. Jackson, C. L. Lewis, S. K. Doorn, V. Majidi, F. L. King, Spectral, spatial, and temporal characteristics of a millisecond pulsed glow discharge: metastable argon atom production, Spectrochim. Acta, 56B (2001) 24492464.

31. C. L. Lewis, G. P. Jackson, S. K. Doorn, V. Majidi, F. L. King, Spectral, spatial, and temporal characteristics of a millisecond pulsed glow discharge: copper analyte emission and ionization, Spectrochim. Acta, 56B (2001) 487-501. 
Chapter 4

Direct Chromium Speciation in Solid State Materials - A GDMS

Approach 


\section{Abstract}

Among the various elemental mass spectrometry techniques, glow discharge mass spectrometry is recognized for its ability to provide direct determination of trace elements present in solid state samples. In the present work, a pulsed glow discharge time of flight mass spectrometry method is developed for the direct speciation of chromium in solid state samples. The millisecond pulsed glow discharge is a versatile ion source that provides elemental, structural and molecular information. Careful tuning of the operating parameters yields the plasma chemistry that favors cluster ion formation. Unique mass spectral features permit differentiation between the trivalent and hexavalent forms of chromium, (CrllI) and (CrVI) respectively, in chromium oxide samples. Specifically, signals at 104 and $120 \mathrm{~m} / z$ corresponding to the $\mathrm{Cr}_{2}{ }^{+}$and $\mathrm{Cr}_{2} \mathrm{O}^{+}$ cluster ions are indicative of the presence of $\mathrm{Cr}(\mathrm{III})$ in the sample, whereas signal at $100 \mathrm{~m} / \mathrm{z}$ corresponding to the $\mathrm{CrO}_{3}{ }^{+}$cluster ion is indicative of the presence of $\mathrm{Cr}(\mathrm{VI})$. The impact of glow discharge operating conditions on the appearance of these characteristic cluster ions is discussed. 


\subsection{Introduction}

Historically, a trace element has been defined as an element with an average concentration of less than $100 \mathrm{ppm}$ in a given sample. However, the definition of a trace element depends on the substance being analyzed, as Paracelsus Von der Besucht stated "All substances are poisons... The right dose differentiates a poison from a remedy". Furthermore, technological advances in specificity and sensitivity have made the analysis of concentrations in the ppb and ppt range quite common. The term dates back to the early $20^{\text {th }}$ century, when scientists were able to detect minute amounts of several elements in living organisms, often describing them as being present in "traces" or "trace amounts" [1]. Throughout the next half century, all efforts to analyze trace elements were focused on total concentrations [2]. Elemental speciation didn't come to the forefront of the scientific community until the late 1950's and early 1960's with the methyl mercury poisoning epidemic of the Japanese residents near Minamata Bay. Subsequent anthropogenic pollution incidents, such as oyster extinctions in Arachon Bay, France due to tributyltin antifouling paints and globalized lead pollution from antiknock agents in gasoline, further demonstrated the need for analytical methodologies to detect and identify specific chemical forms of trace elements [3].

Elemental speciation seeks to identify, characterize, and quantify the defined species, forms, or phases of an element present in a given sample and its distribution [4]. The importance of elemental speciation lies in the fact that environmental changes influence the behavior of both essential and toxic elements through alterations of the forms in which they occur. These forms, 
including oxidation state and the associated complex or molecular form, profoundly impact the toxicity, bioavailability, metabolism, transport, uptake, and distribution of the element [5]. Chromium is just such an example, the toxicity of which is oxidation state dependent. Whereas the trivalent species of chromium (CrIII) is an essential nutrient needed in ultra-trace quantities (50 to $200 \mu \mathrm{g}$ ), the hexavalent species (CrVI) is a known carcinogen and is toxic to humans $[6,7]$. By understanding the most important forms of an element and the transformation processes between those forms, one can more accurately predict their environmental impact. It is for this reason that the relevance of speciation science spans many different scientific backgrounds: specialists in chemistry, biology, geology, physics, nutrition, and medicine all have a need for this kind of information [4].

A speciation study of a given material might include the determination and quantification of the elements present, as well as identification of the chemical nature or molecular form of each species. Such analysis typically requires a plethora of instrumental techniques, sample preparation procedures, and chemical analysis methods [8]. The most common approach to elemental speciation is the utilization of chromatographic separation methods coupled with elemental or molecular characterization techniques such as atomic spectroscopy or mass spectrometry [9]. However, as Marcus states "the greatest level of specificity comes from methods that allow the unambiguous identification of a complete analyte entity" including molecular weight as well as "selective information related to molecular structure (i.e., fragmentation patterns)" [10]. 
In recent years, mass spectrometry (MS) has proven to be the best detection choice for speciation analysis because it provides both the sensitivity and selectivity needed to asses the molecular, structural, or elemental composition of a given analyte. Nevertheless, the type of elucidation achieved by MS is largely contingent on the ionization method used. "Hard" ionization methods like inductively coupled plasma (ICP) provide information regarding the elemental composition, whereas methods like electrospray ionization (ESI), a "soft" ionization technique, allow for the detection of intact molecular ions. As a result, to achieve a complete characterization of a given sample, one must interface among multiple detection methods inevitably increasing the time, cost, sample size and operator skill needed to conduct the analysis [9].

Classic chromatographic and 'wet chemistry' techniques utilized in elemental speciation further complicate the analysis. Because these techniques require liquid samples, many complicated pretreatment procedures, such as extraction, preconcentration, or derivatization are needed. These methods not only lend to additional analysis duration and solvent cost, but also open the possibility for oxidation or reduction reactions to occur, thereby altering the speciation of the original sample [11]. For the analysis of geological samples where the specificity of a particular elemental valency is of great concern, it is often necessary to avoid such modifications and preserve the sample in its natural condition, thus making liquid techniques obsolete [3].

It is the combination of these two pressing needs, a mass spectrometric ionization source that can provide concurrent elemental, structural, and 
molecular information and the necessity for a direct solid sampling technique, that has led our group to investigate the use of a glow discharge time of flight mass spectrometry method for the direct speciation of chromium in solid state samples. Glow discharge (GD) plasmas are "steady-state but highly heterogeneous plasmas with a number of dynamic processes occurring simultaneously" [8]. The atomization of an analyte in a GD ion source arises through cathodic sputtering, in which ionized discharge gas atoms, created by the breakdown of the discharge support gas, are accelerated toward the cathode where they bombard the surface and "knock off" sample atoms. Once atomized, the sputtered analyte atoms can become excited or ionized in the surrounding plasma via interactions with numerous energetic species such as fast electrons, excited and metastable atoms, and ions generated in plasma sustaining processes. Each species transfers energy to analyte molecules through a characteristic ionization process that can excite them within a particular energy range. These processes include electron ionization, charge transfer, and Penning ionization [9].

When operated in the pulsed mode, spatial and temporal separation of the different ionization mechanisms becomes possible through the creation of three distinct time regimes during each on/off cycle: a prepeak dominated by electron ionization, an afterpeak dominated by Penning ionization, and a steady-state plateau which arises from a mixture of electron impact, charge transfer, and Penning ionizations [12]. As a consequence, analyte molecules are exposed to a series of transient ionization periods within each pulse cycle that range from 
"hard" to "soft", allowing for a highly tunable ion source [9]. By coupling a pulsed GD plasma ion source with time-of-flight mass spectrometry (Pulsed-GDMS) and selecting a particular time regime/plasma chemistry, elemental and/or molecular information may be obtained regarding a sample's composition.

The use of GD plasma is now well established for the direct determination of trace elements in solid state samples, including geological materials [13, 14, 15]. Previous research from this lab has also demonstrated the ability of this technique to be modified by time gating to permit chemical speciation of volatile organic and organometallic compounds $[8,9,12]$. In the present work, a PulsedGDMS method for the direct speciation of chromium in solid state samples is presented. Based on similar work conducted by Aubriet et al. utilizing secondary ion mass spectrometry (SIMS) [11, 16], unique mass spectral features have been found that permit differentiation between the trivalent and hexavalent forms of chromium, (CrIII) and (CrVI) respectively, in chromium oxide samples. 


\subsection{Experimental}

The pulsed glow discharge mass spectrometry system employed in these investigations, Figure 1, consisted of the glow discharge ion source and chamber into which the sample materials were introduced, a linear time-of-flight (ToF) oriented orthogonally to the glow discharge ion beam, and associated electronics as described below. Experimental conditions can be found in Table 4.1.

\begin{tabular}{ll}
\multicolumn{2}{l}{ Table 4.1. ToF-MS operating parameters } \\
\hline Glow discharge \\
Pressure & $0.3-0.4$ Torr \\
Operating power & $100-130 \mathrm{~W}$ \\
Pulse duration & $5 \mathrm{~ms}$ \\
Duty cycle & $25 \%$ \\
$\quad$ Sampling distance & $5-9 \mathrm{~mm}$ \\
Time-of-Flight & \\
Flight path length & $1 \mathrm{~m}$ \\
lon lenses potential & \\
$\quad$ Skimmer & $-353 \mathrm{~V}$ \\
Accelerator (A2) & $-1535 \mathrm{~V}$ \\
Deflector (X1, X2) & $-1800 \mathrm{~V}$ \\
Deflector (Y1, Y2) & $-1549 \mathrm{~V}$ \\
Repeller (A1) & $+250 \mathrm{~V} ; 1 \mu \mathrm{s}$ \\
Detector & $-1850 \mathrm{~V}$
\end{tabular}




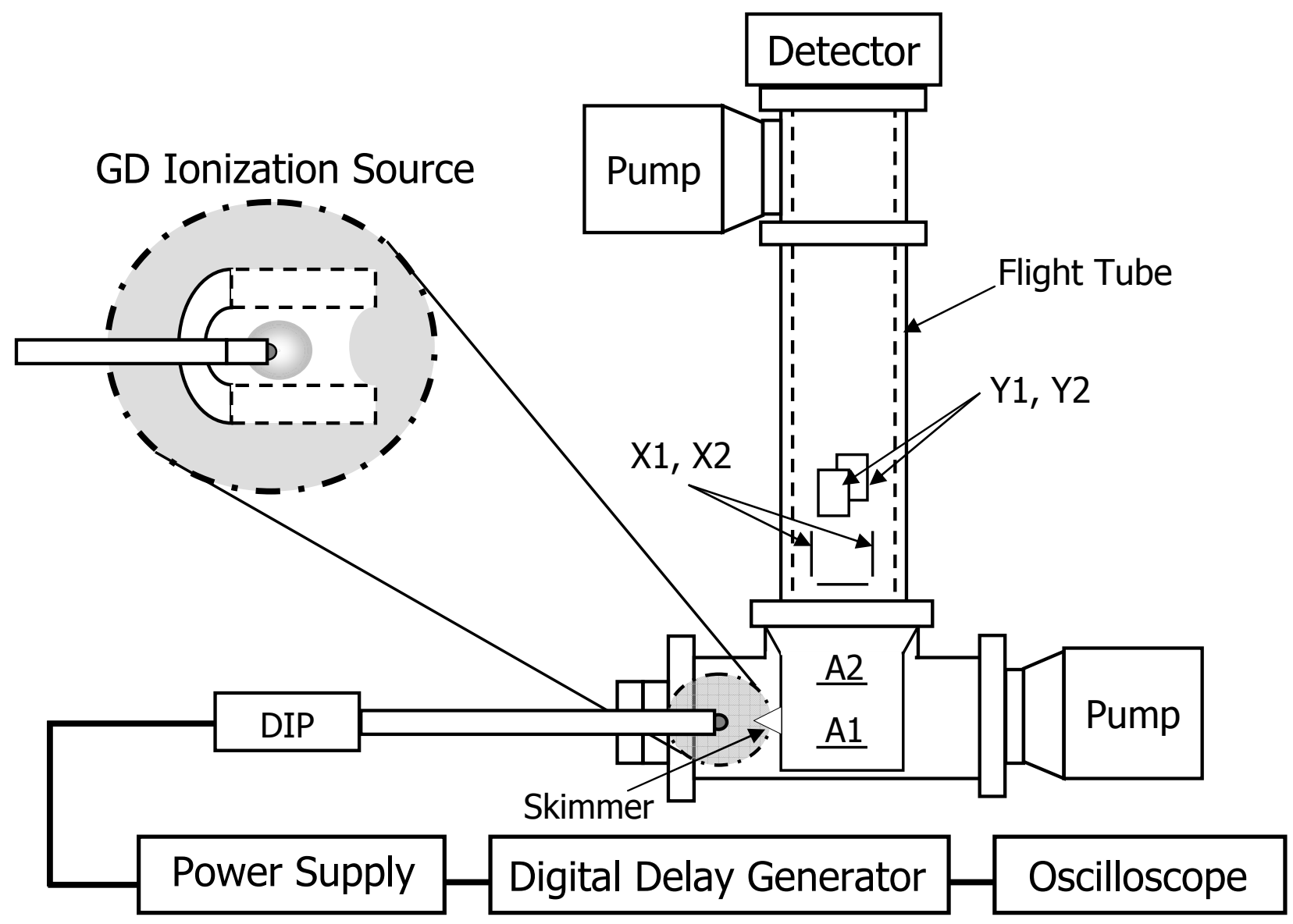

Figure 4.1. Schematic of the GD-ToFMS Instrument (refer to Table 1 for component descriptions). 


\subsubsection{Pulsed Glow Discharge Plasma Device}

A $13.56 \mathrm{MHz}$ radio frequency (rf) generator coupled with an automatic matching network (RF Plasma Product Inc., Marlton, NJ) was used to power the GD plasma. The internal pulsing mode of the if power supply system permits control of applied power, pulse width, and duty cycle. A $5 \mathrm{~ms}$ pulse width with a $25 \%$ duty cycle was maintained throughout the experiments while the operating power was varied. The argon discharge support gas (ultra pure, Airgas, Randor, PA) was introduced through a metering valve to control the gas pressure which was monitored by a thermocouple pressure gauge (Varian, Lexington, MA).

\subsubsection{Chromium Sample Cathode Production}

Analytical reagent grades of chromium (III) oxide $\left(\mathrm{Cr}_{2} \mathrm{O}_{3}\right)$ and chromium (VI) oxide $\left(\mathrm{CrO}_{3}\right)$ were purchased from commercial suppliers (J.T. Baker Chemical Co., Phillipsburg, N.J. and Alfa Aesar, Ward Hill, M.A. respectively). Because the chromium oxide samples do not form sturdy disk samples when pressed, they were mixed with spectroscopic grade silver or graphite powders (Alfa Aesar, Ward Hill, M.A. and Bay Carbon, Bay City, M.I. respectively). The silver matrix adds stability and makes the resulting pellets more conductive. The samples were prepared according to methods developed by this group $[17,18]$. All constituent powders were dried overnight at $90^{\circ} \mathrm{C}$ and stored in a desiccator until needed. Sample weights between 1 to $2 \mathrm{~g}$ were prepared to yield the desired \%weight compositions. The resulting powder mixture was then homogenized in a ball-in-vial mixer (Wig-L-Bug, SPEX, Edison, N.J.) for 3 minutes. Approximately $160 \mathrm{mg}$ of powder was compacted in a die assembly 
constructed in this department's machine shop under $4,000 \mathrm{lbs}$ of force for 30 minutes. The resulting disk-shaped sample pellets were $5 \mathrm{~mm}$ in diameter and $4.5 \mathrm{~mm}$ in height.

\subsubsection{Time-of-Flight Mass Spectrometer}

Instrumentation: The mass spectrometer employed in this study was a linear ToF instrument (R.M. Jordan Co., Grass Valley CA) oriented orthogonally to the glow discharge ion beam. A six-way, high vacuum cross (MDC Vacuum Products Co., Hayward, CA) served as the GD chamber. Plasmas were generated using a direct insertion probe (DIP) onto which the disk-shaped sample cathodes, $5 \mathrm{~mm}$ in diameter and $4.5 \mathrm{~mm}$ in height, were mounted (Figure 2). The DIP allows for axial movement inside the GD chamber enabling ToF sampling at different distances from the cathode surface (Figure 3). Using a nonconducting MACOR $®$ shield (Accuratus, Washington, N.J.), constant cathode surface area and current density were maintained allowing only the face of the sample disk to be exposed to discharge sputtering. Further information on the design of this instrumentation can be found in previous papers published by this group [19]. 
Compacted Pellet

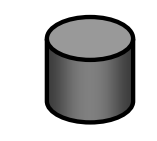

Pellet Holder
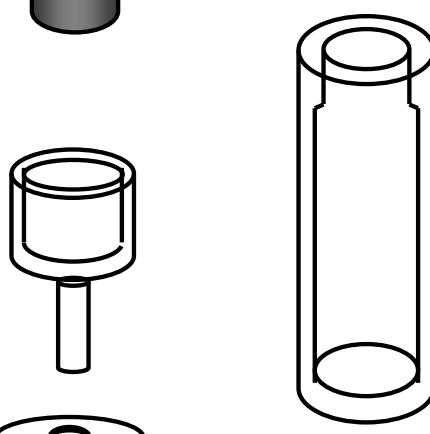

Push-on Connector

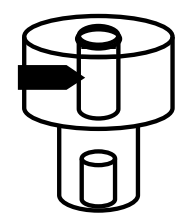

MACOR $®$ Shield

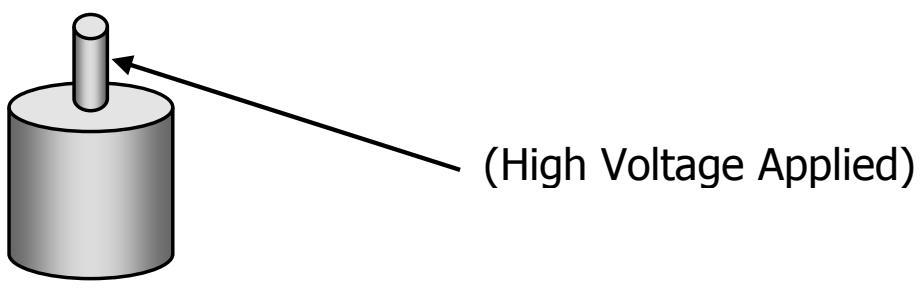

Direct Insertion Probe

Figure 4.2. Schematic of the pellet holder used for mounting compressed sample pellets to the DIP [adapted from 22]. 


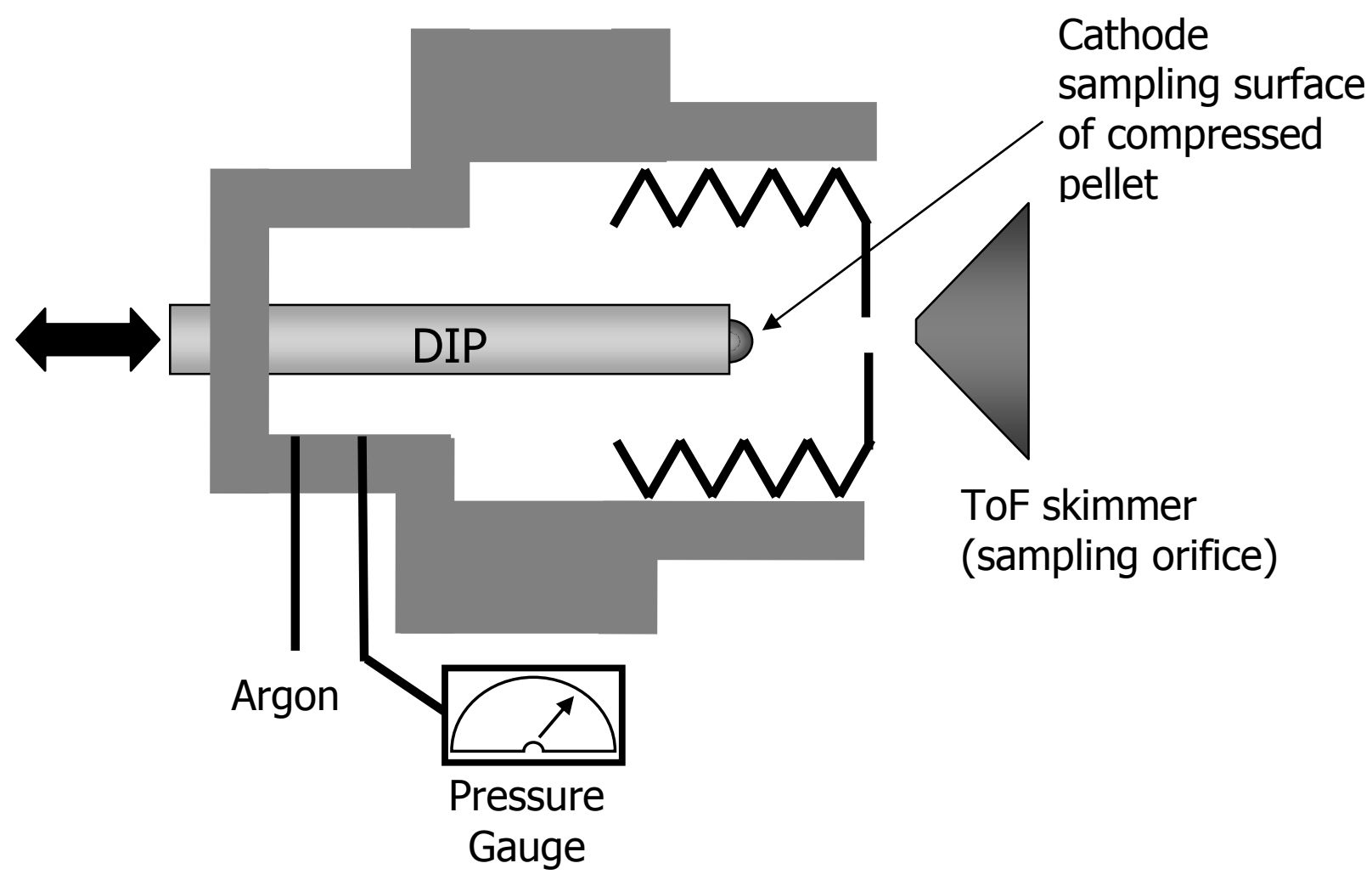

Figure 4.3. Schematic of the GD ion source. 
Data acquisition: A digital delay generator (DDG) (EG\&G Princeton Applied Research, Princeton, NJ) in synchronization with the if power pulse was used to trigger an ion extraction pulse to the ToF repeller thereby initiating the signal detection system. The DDG allows for time-gated detection with which mass spectra can be obtained at selected temporal intervals within the power pulse sequence (Figure 4). In this study, plateau and afterpeak spectra were collected between $4.8 \mathrm{~ms}$ and $5.3 \mathrm{~ms}$ from the pulse initiation. The mass spectrometric data was amplified and fed into a 1-GHz oscilloscope (Lecroy 9370M, Chestnut Ridge, NY) for collection. The spectra were averaged continuously at 1:1023 and transferred to the computer through a GPIB card where a macro tool was used to open the ASCII data in Microsoft Excel and produce the corresponding mass spectrum. 


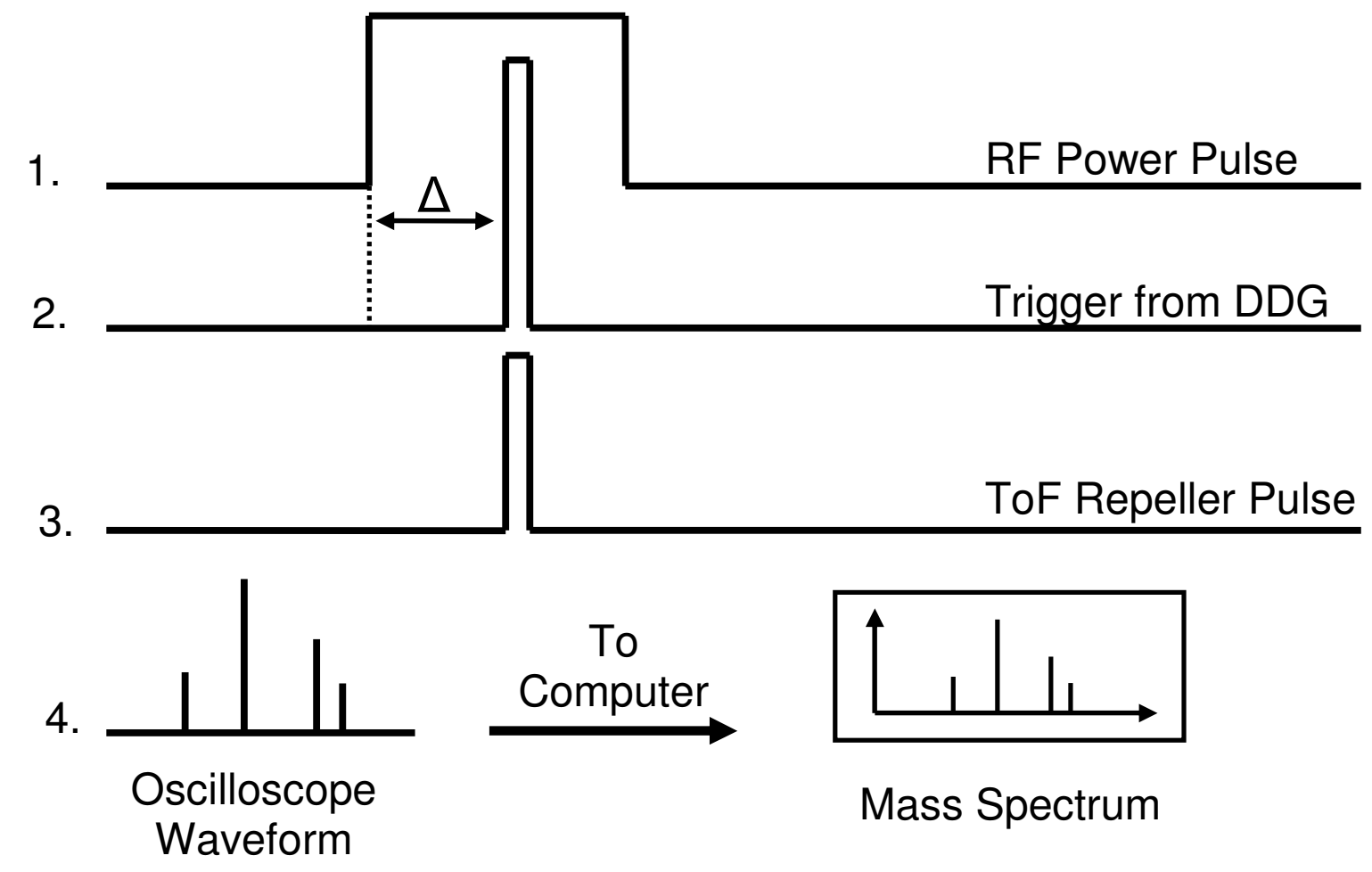

Figure 4.4. Schematic of the time-gated detection and data acquisition system for the ToFMS instrument. The digital delay generator (DDG) allows the trigger delay time $(\Delta)$ to be adjusted relative to the rf power onset. 


\subsection{Results and Discussion}

\subsubsection{Evaluation of Temporal Regimes}

The majority of analytical glow discharge (GD) plasmas operate in a continuous power mode, generating steady-state analytical signals. When operated in a modulated or pulsed power mode, several unique characteristics are imparted to the plasma. These characteristics contribute to significant analytical advantages for pulsed GD plasmas over their continuous counterparts. First, because each "power-on" period is followed by a much longer "power-off" period allowing time for the cathode to cool, higher instantaneous power can be applied to the plasma to enhance sputtering, excitation, and ionization while preventing problems associated with sample overheating. Second, pulsed operation of the plasma enables temporal discrimination between analyte signal intensities and those of the discharge gas and background contaminants through the creation of three distinct time regimes, the prepeak, plateau, and afterpeak [19]. Within each pulse cycle analyte molecules are exposed to a series of transient ionization periods: a prepeak dominated by electron ionization, an afterpeak dominated by Penning ionization, and a steady-state plateau which arises from a mixture of electron impact, charge transfer, and Penning ionizations [12]. Such temporal discrimination allows for a highly tunable ion source offering windows of ionization that range from "hard" to "soft." In the present study, it is the unique plasma chemistry of the afterpeak, the period immediately following the discharge power termination during which a large population of metastable argon species is created, that is of greatest interest. 
Upon power termination, high-energy electrons rapidly lose kinetic energy through collisional processes in the GD chamber. Although these thermalized electrons are no longer sufficiently energetic enough to ionize gas-phase species, they can recombine with argon ions to form energetic metastable atoms [20]. Metastable atoms efficiently ionize sputtered analyte atoms or molecules of lower ionization potentials through a process called Penning lonization. During this process, collisions between metastable and analyte atoms result in the transfer of the metastable atom's potential energy, 11.55 and $11.62 \mathrm{eV}$ for metastable argon atoms, to form an analyte ion and a ground state argon atom. Because Penning ionization is limited by the relatively low potential energies of the metastable argon atoms, molecular fragmentation is inhibited and thus the afterpeak exhibits enhanced signals for the molecular ions sputtered from the analyte material [8].

Temporal comparisons were made between a pure silver pellet and a mixed pellet with $20 \%$ hexavalent chromium oxide $\left(\mathrm{CrO}_{3}\right)$ and $80 \%$ silver by weight (Figures 4.5 and 4.6). In each case, the GD plasma was operated at 120 W rf power and 0.32 torr argon discharge gas with the DIP positioned such that the cathode was $9 \mathrm{~mm}$ from the sampling orifice. Time resolved mass spectra were collected within each region of the pulse cycle for both pellets: prepeak $(0.3$ and $0.4 \mathrm{~ms})$, plateau $(4.9 \mathrm{~ms})$, and afterpeak $(5.1 \mathrm{~ms})$.

Prepeak: Just after the onset of power, 0.3 and $0.4 \mathrm{~ms}$ into the pulse, electron ionization dominates as electrons are accelerated away from the cathode and interact with the discharge gas. At this time, the argon ion profile 
maximizes. Minimal signal contributions are obtained from the sample species, silver and chromium, due to delays in analyte sputtering and diffusion into the negative glow region of the plasma.

Plateau: At approximately $1 \mathrm{~ms}$ into the pulse cycle and until power termination, the plasma reaches pseudo steady-state conditions. In this temporal region, ionization mechanisms equilibrate into a mixture of electron impact, charge transfer, and Penning ionizations. Mass spectra collected in this region tend to be highly fragmented, yielding structural information about the analyte. Plateau mass spectra were obtained at $4.9 \mathrm{~ms}$ into the pulse cycle. Signal contributions from both the argon discharge gas and cathode material are present. For the mixed chromium $(\mathrm{VI})$ oxide and silver pellet, steady-state signals were obtained for the ions of $\mathrm{Cr}, \mathrm{CrOH}, \mathrm{CrO}_{2} \mathrm{H}$, and the isotopes of $\mathrm{Ag}(\mathrm{m} / \mathrm{z} \mathrm{52}$, $69,85,107$, and 109 respectively).

Afterpeak: At $0.1 \mathrm{~ms}$ after power termination $(5.1 \mathrm{~ms}$ into the pulse cycle), analyte ion signals maximize while the intensities of discharge species and background contaminants wane. As stated earlier, this temporal region is dominated by Penning ionization through a large population of metastable argon ions. For the pure silver pellet, the 107 and 109 masses maximize and saturate the detector. The silver dimer (m/z 214) and water-silver complexes ( $\mathrm{m} / \mathrm{z} 125$ and 127) can also be seen. For the mixed chromium ( $\mathrm{VI}$ ) oxide and silver pellet, the ion signal intensities for $\mathrm{Cr}, \mathrm{CrO}, \mathrm{CrOH}, \mathrm{CrO}_{2}$ and $\mathrm{CrO}_{2} \mathrm{H}$ increase $(\mathrm{m} / \mathrm{z} 52,68$, 69,84 , and 85 respectively) and the parent ion appears $\left(\mathrm{CrO}_{3} \mathrm{H}, \mathrm{m} / \mathrm{z} 101\right)$. 
Because this project desires the direct speciation of solid state samples to permit differentiation between the trivalent and hexavalent forms of chromium, the afterpeak time regime was chosen as the most analytically favorable for chromium cluster ion formation. All further mass spectra for chromium oxide and silver pellets were obtained at $5.1 \mathrm{~ms}$. For the mixed pellets of chromium oxide and graphite, $5.3 \mathrm{~ms}$ was found to give the most intense analyte peaks. 


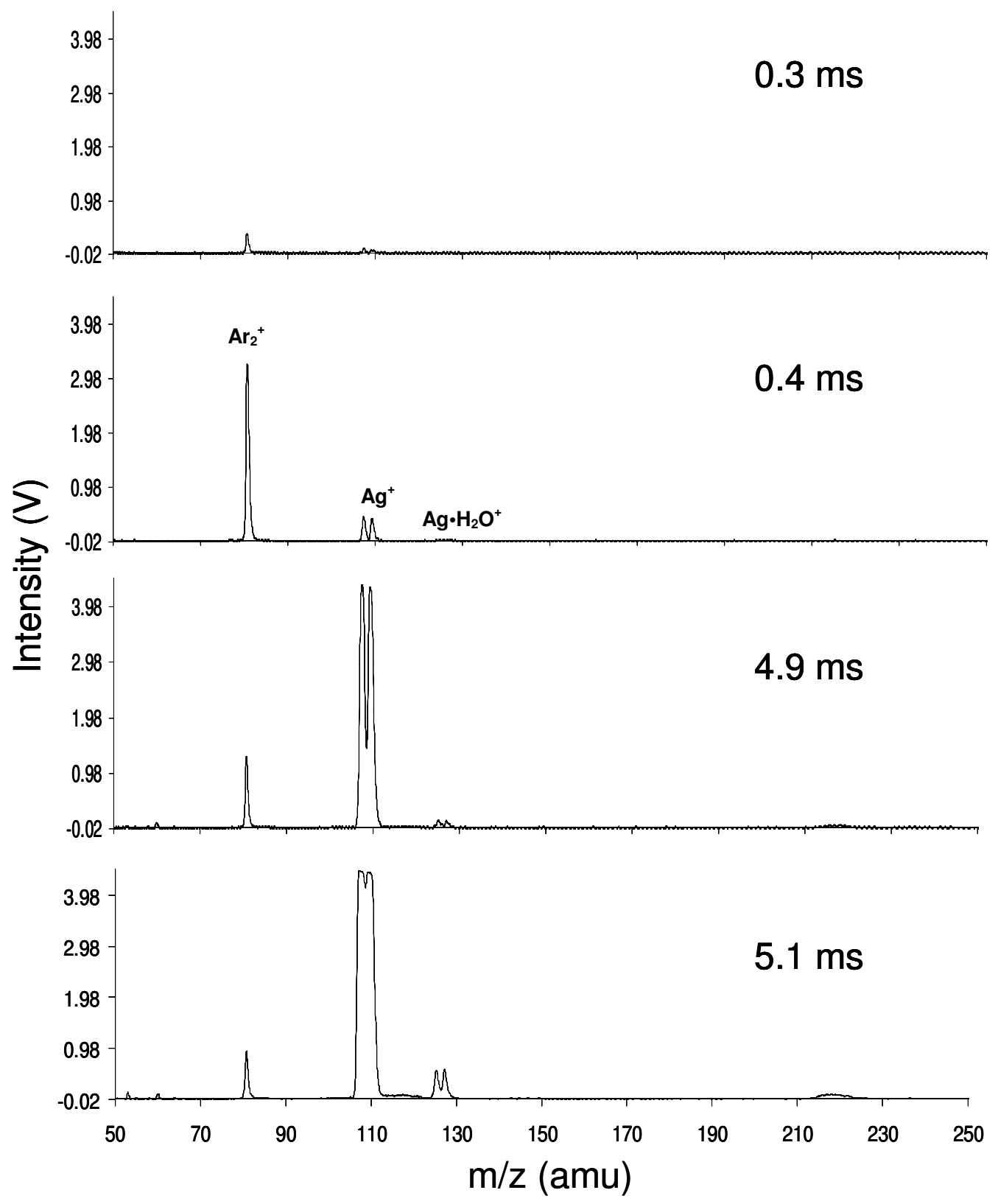

Figure 4.5. Time resolved mass spectra of pure silver during the prepeak (0.3 $\mathrm{ms}$ and $0.4 \mathrm{~ms})$, plateau $(4.9 \mathrm{~ms})$, and afterpeak $(5.1 \mathrm{~ms})$. Discharge pressure 0.32 torr, operating power $120 \mathrm{~W}$, and sampling distance $9 \mathrm{~mm}$. At $5.1 \mathrm{~ms}$ the ion intensity of the silver line becomes saturated. 


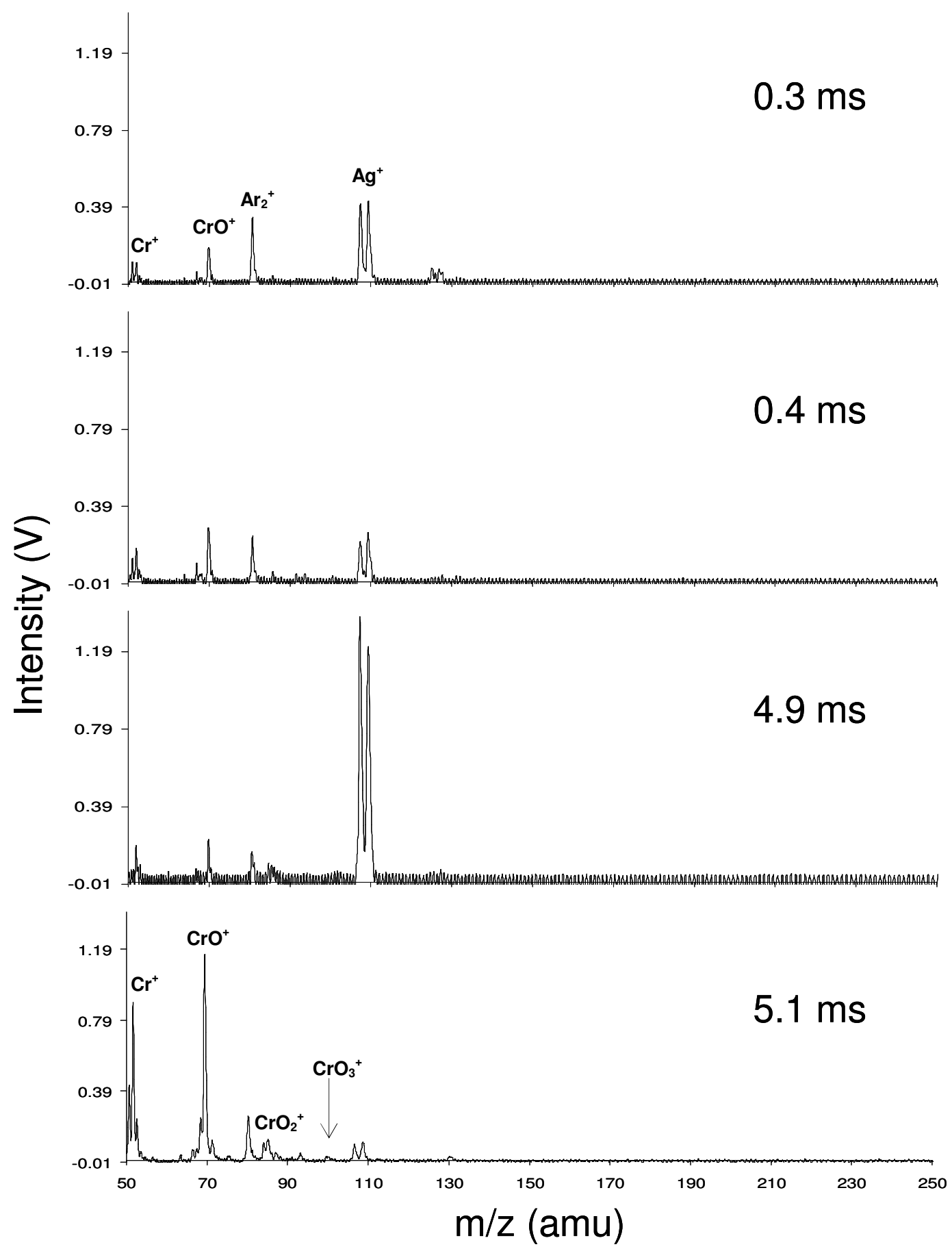

Figure 4.6. Time resolved mass spectra of $20 \%$ chromium (VI) oxide and silver pellet during the prepeak ( $0.3 \mathrm{~ms}$ and $0.4 \mathrm{~ms})$, plateau $(4.9 \mathrm{~ms})$, and afterpeak $(5.1 \mathrm{~ms})$. Discharge pressure 0.32 torr, operating power $120 \mathrm{~W}$, and sampling distance $9 \mathrm{~mm}$. 


\subsubsection{Evaluation of Operating Parameters}

One can significantly affect excitation and ionization processes within the GD by controlling the plasma operating parameters. Careful adjustment of the applied power or discharge pressure makes it possible to selectively enhance a particular ionization mechanism thus providing additional tuning capabilities for the optimization of a given analyte signal $[9,12]$. The operating pressure controls the collisional environment of the GD plasma, whereas the applied potential defines the kinetic energy that can be obtained by charged species in the plasma [17]. Because excitation and ionization processes within pulsed GD plasmas show spatial heterogeneity over the pulse cycle, further tuning capabilities can be gained via careful selection of the plasma sampling region. Studies of discharge pressure, operating power, and sampling distance were conducted to favor the formation of chromium cluster ions in the plasma (Figures 4.7, 4.8, and 4.9 respectively). The optimal settings can be found in Table 4.2.

Discharge Pressure: The discharge pressure determines the mean free path and collision frequency of ions and atoms in the plasma. Figure 4.7 shows the effects of discharge pressure on the afterpeak mass spectra of a pellet mixed with $30 \%$ trivalent chromium oxide $\left(\mathrm{Cr}_{2} \mathrm{O}_{3}\right)$ and silver. While higher pressures yielded high intensity peaks for elemental chromium and silver ( $\mathrm{m} / \mathrm{z} 52$ and 107-109 respectively), lower pressures favor the formation of larger chromium clusters. These results are in agreement with previous work conducted by this group. While investigating the molecular energetics of GD-ToFMS using the thermometer molecule tungsten hexcarbonyl, Li et al found that pressures between 0.2 to 0.6 torr were optimal for the "soft" ionization of large molecules. 
Higher pressures lead to extensive fragmentation, while extremely low pressures resulted in low analyte signal intensities [9].

Operating Power: Although the operating power has little effect on the type of ionization garnered in the plasma, it greatly influences sputtering yields and ion intensities. Figure 4.8 shows the effects of operating power on the afterpeak mass spectra of a pellet mixed with $28 \%$ hexavalent chromium oxide $\left(\mathrm{CrO}_{3}\right)$ and silver. Under conditions of constant pressure and distance, increases in operating power yielded increases in ion intensities. This was especially noticeable for chromium oxide clusters, with only modest increases in the ion intensities of elemental chromium and silver.

Sampling Distance: While the optimal spatial distribution from which the GD is sampled is somewhat dependent on the analyte material under investigation, the metastable argon population tends to maximize approximately $5-10 \mathrm{~mm}$ from the cathode surface leading to enhanced molecular ion intensities in this region $[9,21]$. Figure 4.9 shows the afterpeak mass spectra of a $20 \%$ hexavalent chromium oxide $\left(\mathrm{Cr}_{2} \mathrm{O}_{3}\right)$ and silver pellet taken at various distances from the sampling orifice. As the plasma is sampled farther out from the cathode surface, around $9 \mathrm{~mm}$, the ion intensities of chromium and chromium oxide clusters increase dramatically. Distances closer to the cathode (3- $7 \mathrm{~mm})$ show only lower mass chromium clusters, while farther distances (11 and 13 $\mathrm{mm}$ ) have decreased intensities. 


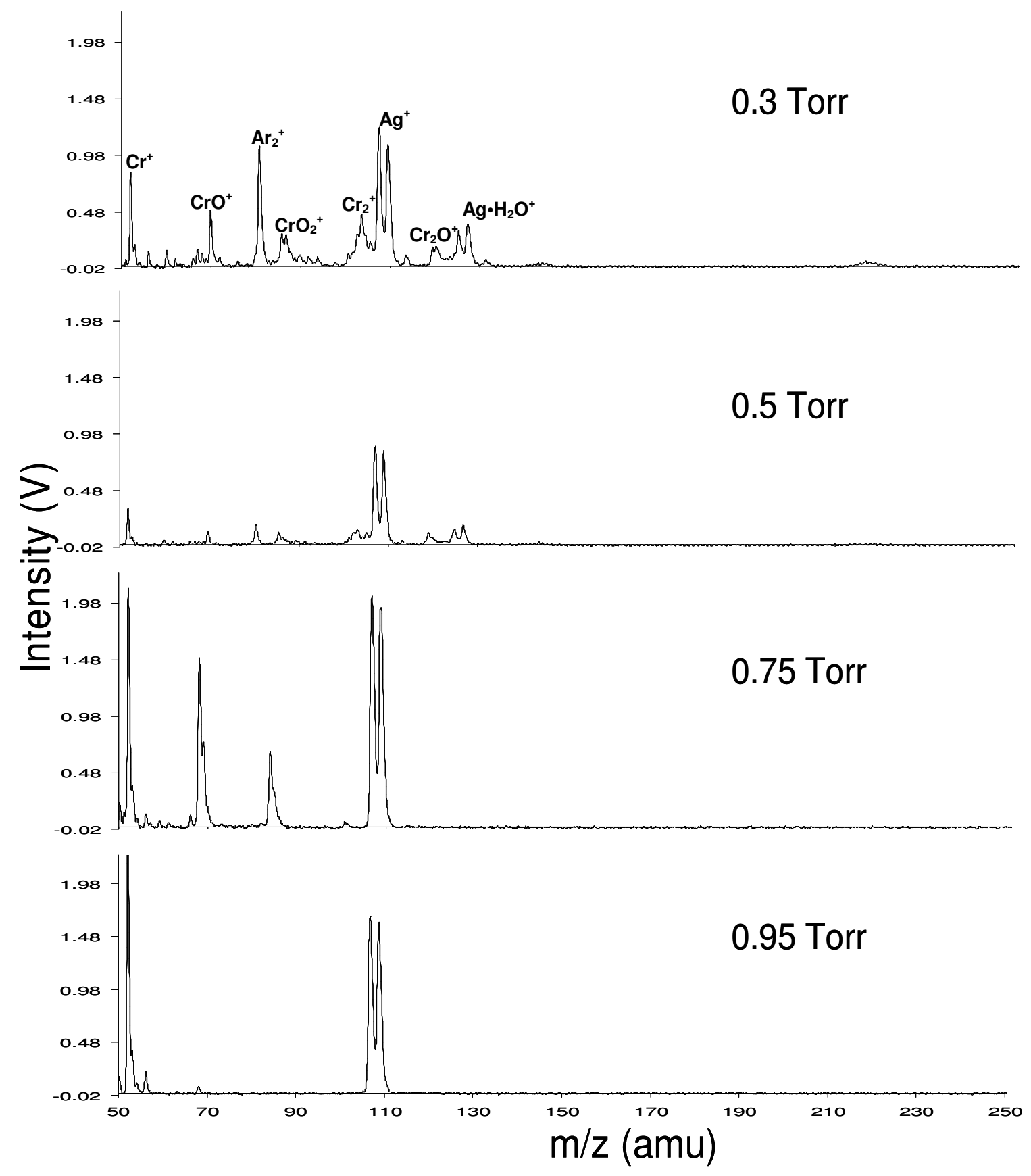

Figure 4.7. Effects of discharge pressure on the afterpeak mass spectra (5.1 ms) of a pellet mixed with $30 \%$ chromium (III) oxide and silver. Operating power $120 \mathrm{~W}$ and sampling distance $9 \mathrm{~mm}$. 


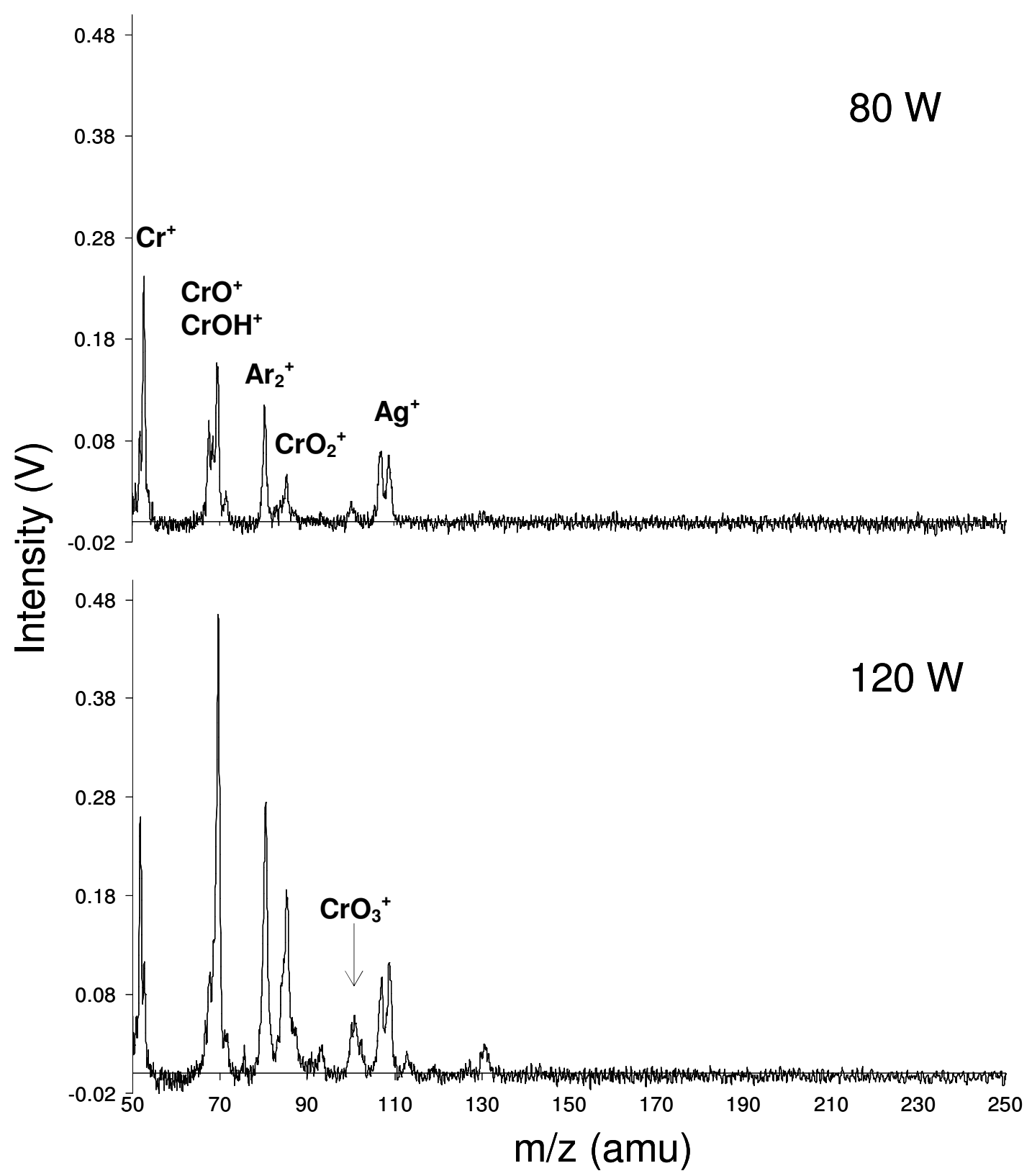

Figure 4.8. Effects of operating power on the afterpeak mass spectra ( $5.1 \mathrm{~ms}$ ) of a pellet mixed with $28 \%$ chromium (VI) oxide and silver. Discharge pressure 0.32 torr and sampling distance $9 \mathrm{~mm}$. 


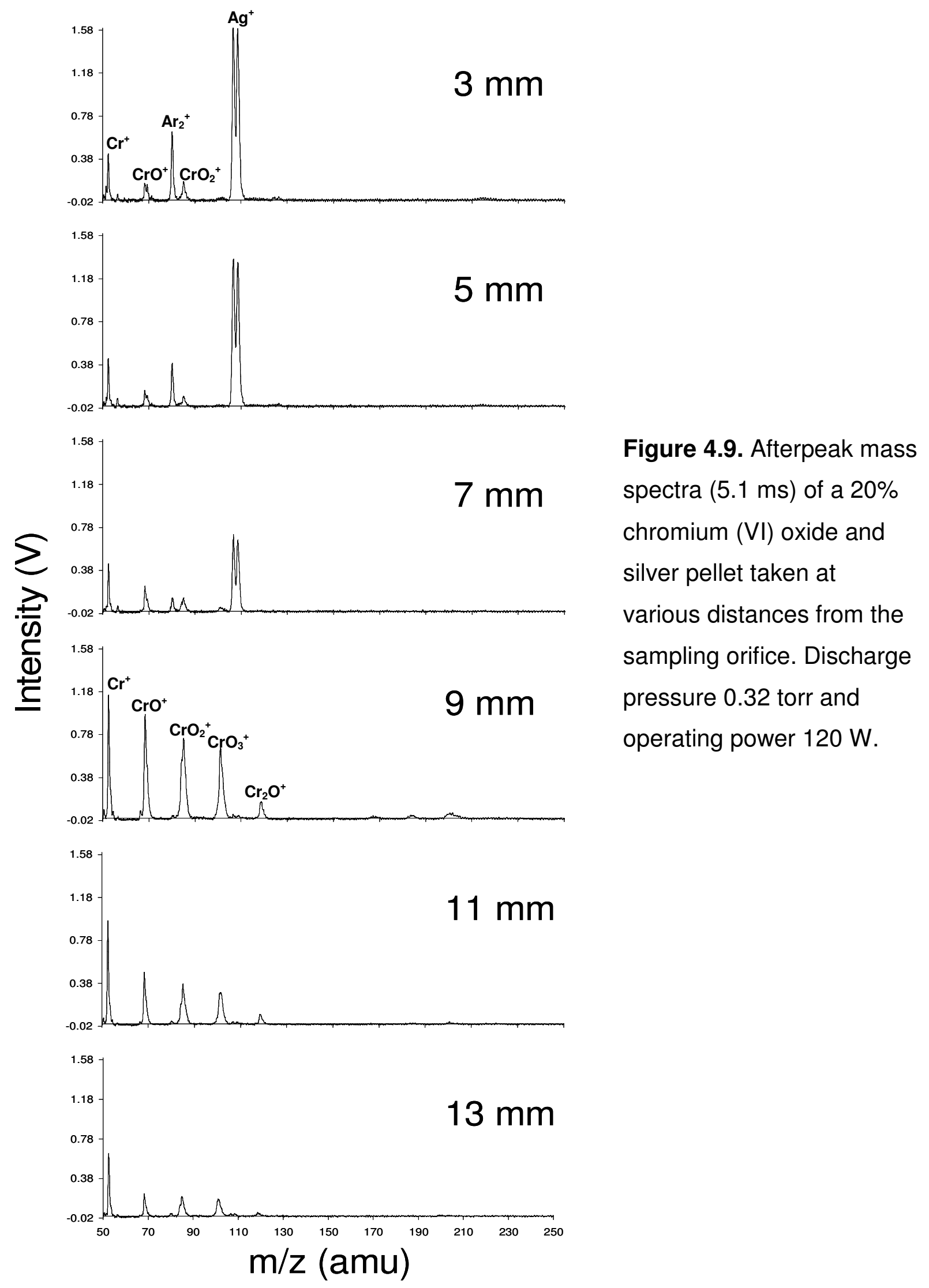


Table 4.2. Plasma Operating Parameters

$\begin{array}{lrrrr}\text { Pellet Type } & \begin{array}{r}\text { Pulse } \\ \text { Time }\end{array} & \begin{array}{r}\text { Discharge } \\ \text { Pressure } \\ 0.32 \text { torr }\end{array} & \begin{array}{r}\text { Operating } \\ \text { Power } \\ 120 \mathrm{~W}\end{array} & \begin{array}{r}\text { Sampling } \\ \text { Distance } \\ 9 \mathrm{~mm}\end{array} \\ \begin{array}{l}\text { Chromium and } \\ \text { Silver }\end{array} & 5.1 \mathrm{~ms} & & & \\ \begin{array}{l}\text { Chromium and } \\ \text { Graphite }\end{array} & 5.3 \mathrm{~ms} & 0.32 \text { torr } & 100 \mathrm{~W} & 5 \mathrm{~mm}\end{array}$

\subsubsection{Speciation and Quantitative Analysis}

Differentiation of Pure Pellets: The mass spectra of hexavalent and trivalent chromium oxide in silver matrices are shown in Figures 4.10 and 4.11 respectively. Several $\mathrm{Cr}_{x} \mathrm{O}_{y}{ }^{+}$cluster ions and hydrogenated species were detected for both chromium oxides, although the number, relative intensity, and distribution vary depending on the particular chromium oxide. Specifically, chromium (VI) oxide produced more numerous and more oxygenated species with molecular ions containing up to four chromium atoms. These findings are in direct agreement with previous chromium speciation work conducted by Aubriet et al using secondary ion mass spectrometry (SIMS) $[11,16]$. The disparity in the number of cluster ions produced may be due to differences in ionization potential (IP), with the IP of hexavalent chromium closely matching that of metastable argon, 11.6 and 11.55 respectively. Specific ions were detected for both chromium oxides. $\mathrm{Cr}_{2}{ }^{+}$and $\mathrm{Cr}_{2} \mathrm{O}^{+}(\mathrm{m} / \mathrm{z} 104$ and 120) were characteristic of trivalent chromium oxide $\left(\mathrm{Cr}_{2} \mathrm{O}_{3}\right)$, while $\mathrm{CrO}_{3}{ }^{+}(\mathrm{m} / \mathrm{z}$ 100) and numerous larger cluster ions, $\mathrm{Cr}_{2-4} \mathrm{O}_{4-12}{ }^{+}(\mathrm{m} / \mathrm{z} 168,184,200,252,268,284,300,352,368,384$, and 400), were specific for the hexavalent chromium oxide $\left(\mathrm{CrO}_{3}\right)$. 
Differentiation of Mixed Pellets: The differentiation between trivalent and hexavalent chromium oxide was also studied using a graphite matrix. Figure 4.12 shows the afterpeak mass spectra of a $20 \%$ chromium (III) oxide and graphite pellet and a $20 \%$ chromium (VI) oxide and graphite pellet. Although no specific peaks were detected for each oxidation state, the relative intensities of certain peaks allows one to easily discriminate between the two forms, in particular, the ratio of the $\mathrm{CrO}_{3}-\mathrm{CrO}_{3} \mathrm{H}$ to $\mathrm{Cr}_{2}$ cations (m/z $100-101$ and 104 respectively). As would be expected, higher ion abundances of the $\mathrm{Cr}_{2}$ ion is indicative of chromium (III) oxide $\left(\mathrm{Cr}_{2} \mathrm{O}_{3}\right)$, while higher ion abundances of the $\mathrm{CrO}_{3}$ ion is indicative of chromium $(\mathrm{VI})$ oxide $\left(\mathrm{CrO}_{3}\right)$. Other specific markers include; m/z 120 - 121, corresponding to the $\mathrm{Cr}_{2} \mathrm{O}$ and $\mathrm{Cr}_{2} \mathrm{OH}$ cations, which are characteristic for the trivalent form, and appreciably higher intensities for $\mathrm{m} / \mathrm{z} 68-69$ and $84-85$, corresponding to the $\mathrm{CrO}, \mathrm{CrOH}, \mathrm{CrO}_{2}$, and $\mathrm{CrO}_{2} \mathrm{H}$ cations respectively, are characteristic of the hexavalent chromium oxide.

Mixed comparisons with varying ratios of the trivalent to hexavalent oxides in graphite were also performed. Figure 4.13 shows the afterpeak mass spectra of a pellet mixed with $15 \%$ chromium (III) oxide, $5 \%$ chromium (VI) oxide, and graphite and a pellet mixed with $5 \%$ chromium (III) oxide, $15 \%$ chromium (VI) oxide, and graphite. Using the same characteristic masses as stated earlier for graphite, one can quickly discern the oxidation state of greater quantity. Again, higher relative ion abundances for masses 104,120, and 121 are indicative of $\mathrm{Cr}_{2} \mathrm{O}_{3}$, while higher relative ion abundances for masses 100 or 101 are indicative of $\mathrm{CrO}_{3}$. 
Quantitative Analysis: Differences in sample preparation, weighing, drying, mixing, and pressing, as well as plasma instabilities, can cause the relative intensities to deviate from a linear response. For this reason, an internal standard is often useful for quantitative analysis where the response from the instrument varies from run to run. Pellets were pressed using a $1 \%$ silver internal standard mixed with varying \% concentrations of trivalent or hexavalent chromium oxide and spectroscopic grade graphite. The average intensities of the silver isotopes, $\mathrm{m} / \mathrm{z} 107$ and 109, were then used to normalize the relative intensities of several chromium and chromium oxide peaks: $\mathrm{Cr}, \mathrm{CrO}_{3}, \mathrm{Cr}_{2}$, and $\mathrm{Cr}_{2} \mathrm{O}$ (m/z 52, 100, 104, and 120 respectively). The average of at least five distinct measurements were calculated and plotted versus concentration. In Figure 4.14, the analytical calibration curves for chromium (III) oxide and chromium (VI) oxide in graphite are shown. The standard deviations between runs were calculated and are included by each point having error bars within the diameter of the marker on the plot. The R-square values for chromium (III) oxide and chromium (VI) oxide were found to be 0.9210 and 0.9222 respectively. 


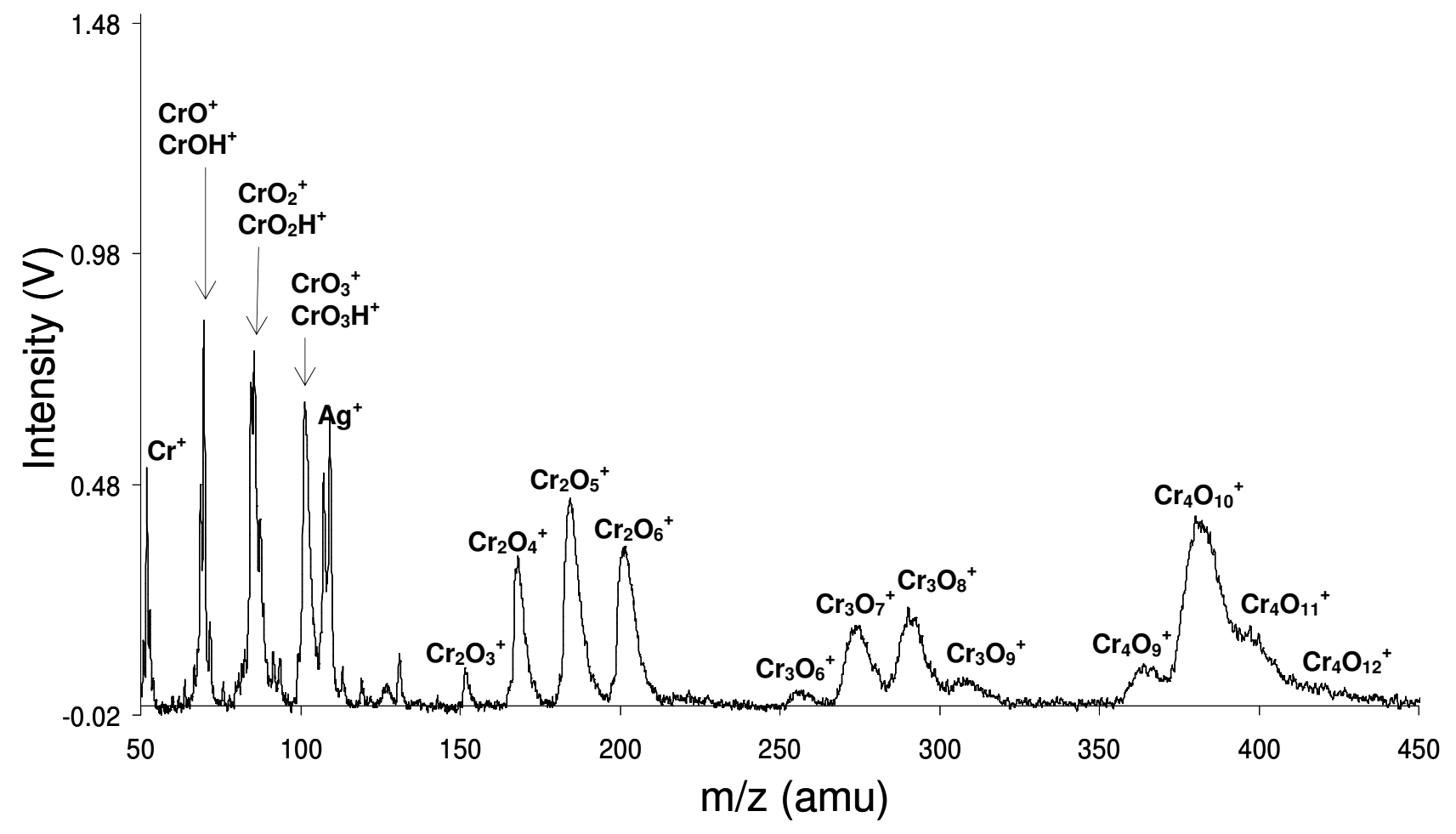

Figure 4.10. Afterpeak (5.1 ms) mass spectra of $20 \%$ chromium (VI) oxide with silver. Discharge pressure 0.32 torr, operating power $120 \mathrm{~W}$, and sampling distance $9 \mathrm{~mm}$. 


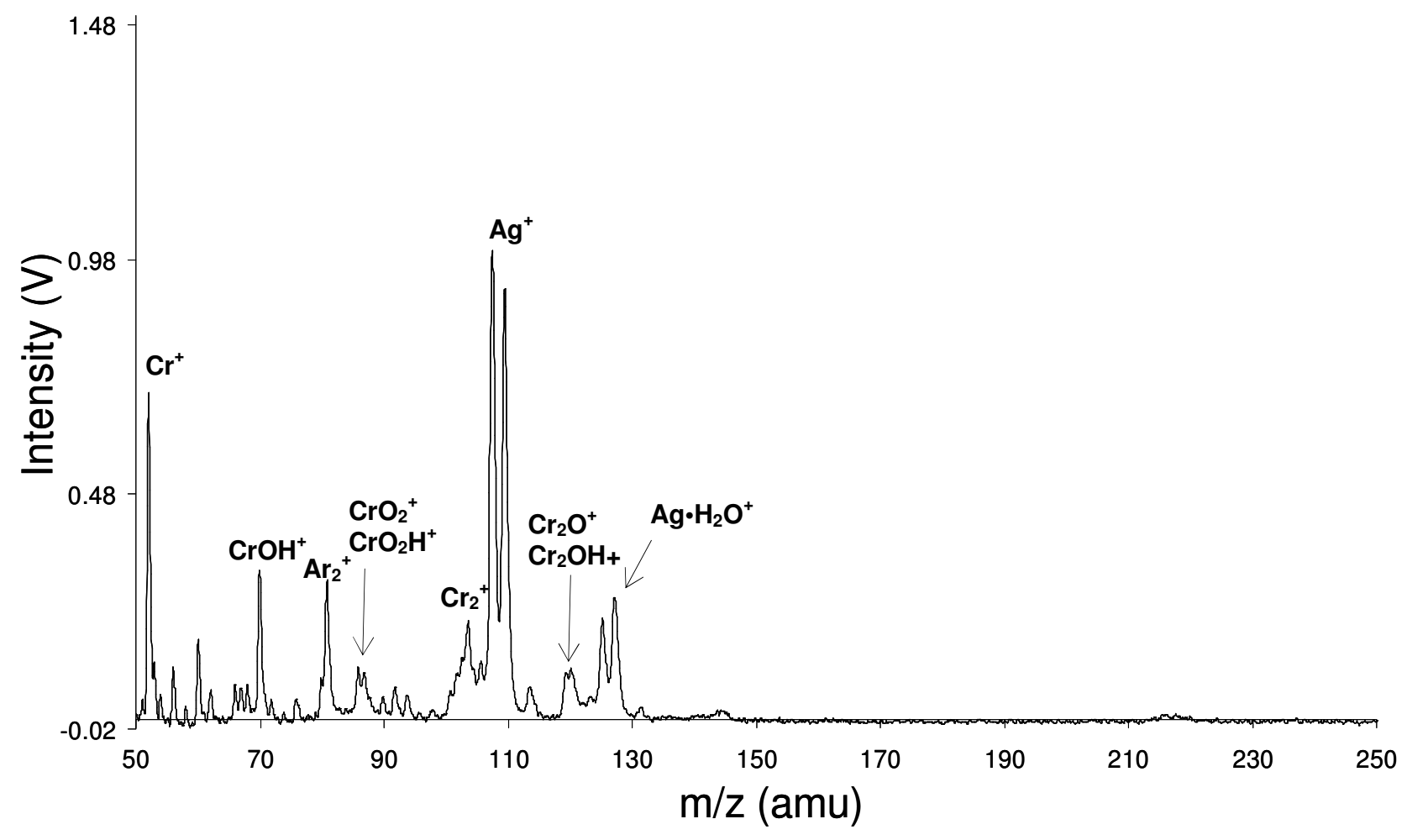

Figure 4.11. Afterpeak (5.1 ms) mass spectra of $30 \%$ chromium (III) oxide with silver. Discharge pressure 0.32 torr, operating power $120 \mathrm{~W}$, and sampling distance $9 \mathrm{~mm}$. 


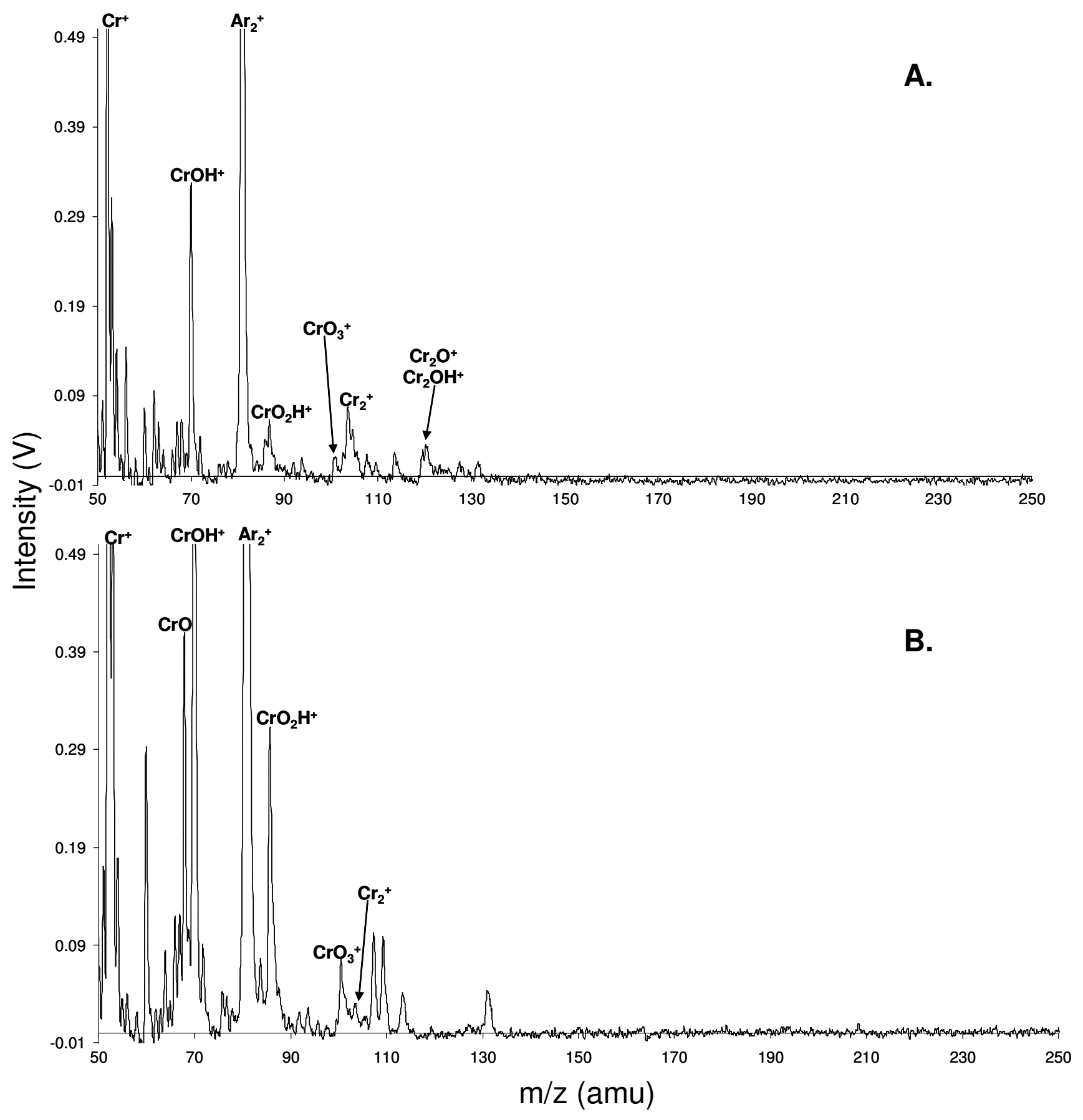

Figure 4.12. Afterpeak (5.3 ms) mass spectra comparison of $(A)$ a $20 \%$ chromium (III) oxide and graphite pellet and (B) a 20\% chromium (VI) oxide and graphite pellet. Discharge pressure 0.32 torr, operating power $100 \mathrm{~W}$, and sampling distance $5 \mathrm{~mm}$. 


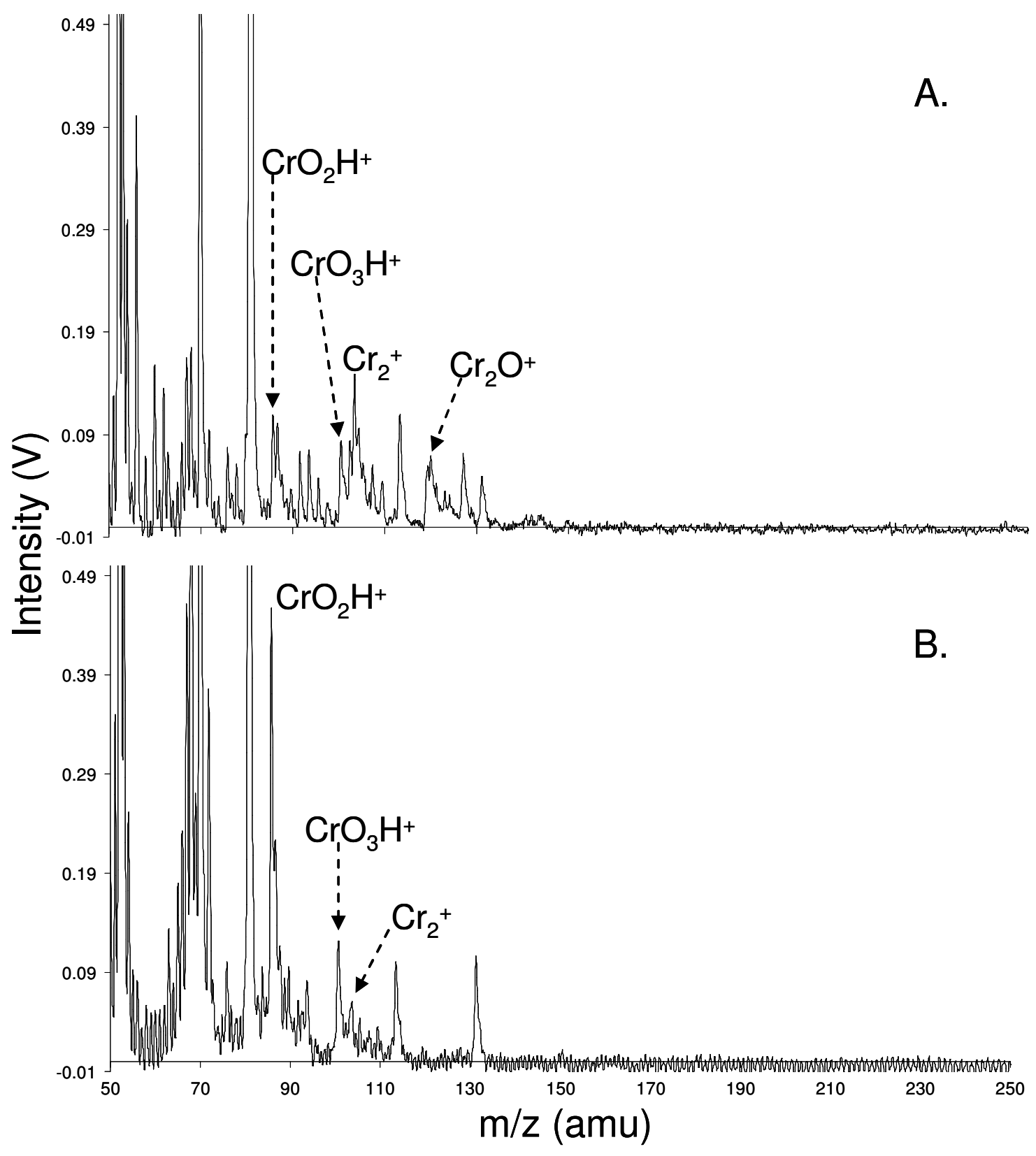

Figure 4.13. Afterpeak (5.3 ms) mass spectra comparison of $(A)$ a pellet mixed with $15 \%$ chromium (III) oxide, $5 \%$ chromium (VI) oxide, and graphite and (B) a pellet mixed with 5\% chromium (III) oxide, 15\% chromium (VI) oxide, and graphite. Discharge pressure 0.32 torr, operating power $100 \mathrm{~W}$, and sampling distance $5 \mathrm{~mm}$. 


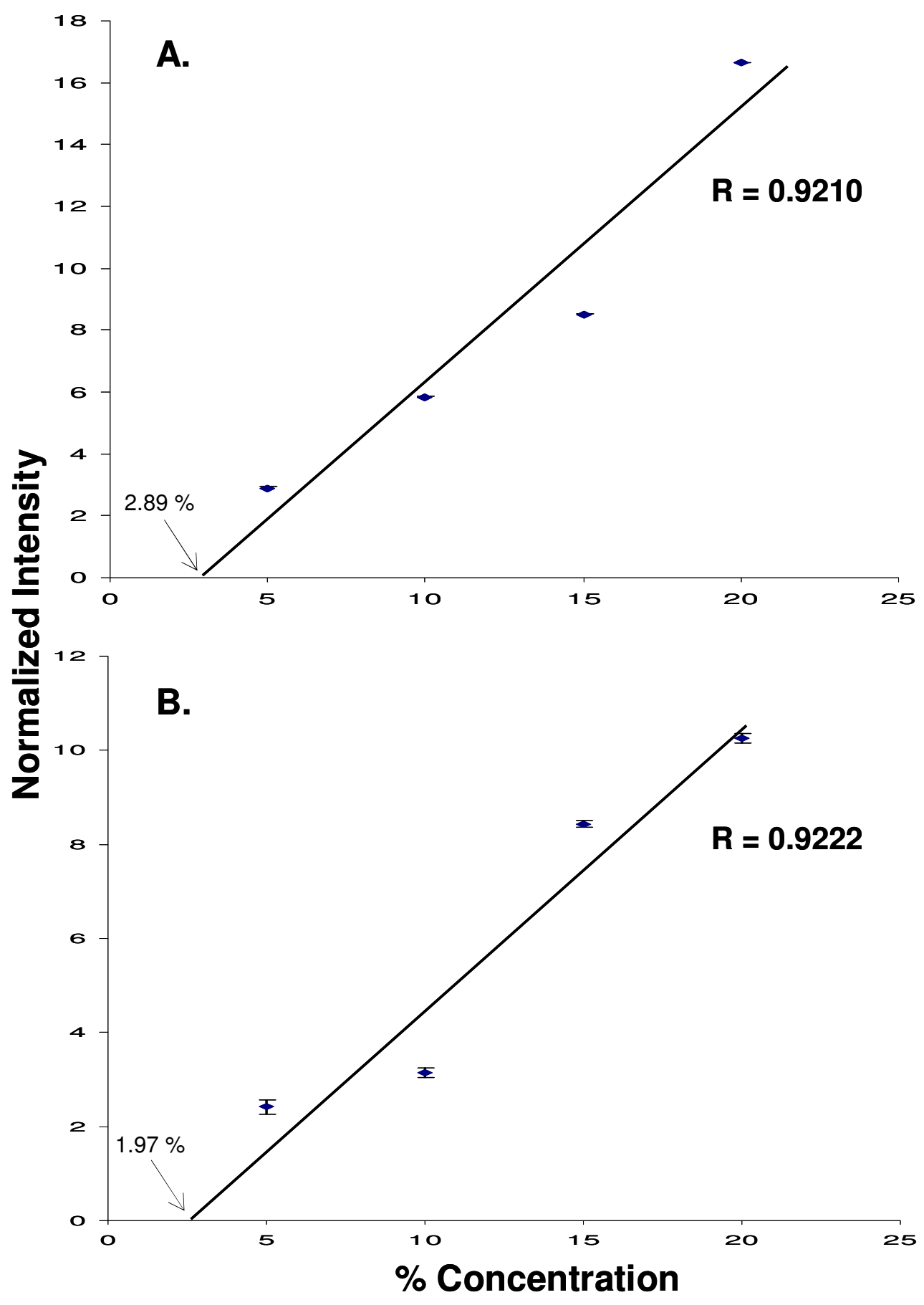

Figure 4.14. Calibration curves of (A) $5,10,15$, and $20 \%$ chromium (III) oxide mixed with graphite and $1 \%$ silver, and (B) 5, 10, 15, and $20 \%$ chromium (VI) oxide mixed with graphite and 1\% silver. Working conditions: 0.32 torr, $100 \mathrm{~W}, 5 \mathrm{~mm}$, and $5.1 \mathrm{~ms}$ into the pulse cycle. 


\subsection{Conclusions}

As with many transition metals, the toxicity of chromium is oxidation state dependent. Whereas the trivalent species of chromium (CrIII) is an essential nutrient needed in ultra-trace quantities, the hexavalent species $(\mathrm{CrVI})$ is toxic to humans and is a known carcinogen. It is for this reason that the ability to distinguish the valency of chromium is of great importance, a task defined under the broad definition of speciation. The data presented in this work demonstrate the suitability of Pulsed-GDMS for the direct speciation of chromium in solid state samples. Chromium (III) and chromium (VI) oxide samples were dried, mixed with matrices of spectroscopic grade silver or graphite by weight, and pressed into pellets. These pellets were then introduced into a pulsed if glow discharge using a direct insertion probe and analyzed by time-of-flight mass spectrometry. Unique mass spectral features were found, permitting differentiation between the trivalent and hexavalent forms of chromium. Specifically signal at 104 and 120 $\mathrm{m} / \mathrm{z}$, corresponding to the $\mathrm{Cr}_{2}{ }^{+}$and $\mathrm{Cr}_{2} \mathrm{O}^{+}$cluster ions, is indicative of the presence of $\mathrm{Cr}(\mathrm{III})$ in the sample, while signal at $100 \mathrm{~m} / \mathrm{z}$, corresponding to the $\mathrm{CrO}_{3}{ }^{+}$cluster ion, is indicative of the presence of $\mathrm{Cr}(\mathrm{VI})$. Discrimination was also achieved in mixed pellets with varying ratios of the trivalent to hexavalent oxides and calibration curves were constructed permitting quantitative analysis of each form in graphite. The R-square values from the calibration curves for chromium (III) oxide and chromium (VI) oxide were found to be 0.92 and 0.94 respectively. 


\subsection{References}

1. "trace element." Encyclopedia of Food and Culture. The Gale Group, Inc, 2003. Answers.com 20 May. 2007. http://www.answers.com/topic/micromineral

2. R. C. Cornelis, H. M. Crews, J. A. Caruso, K. G. Heumann, Handbook of Elemental Speciation: Techniques and Methodology, John Wiley and Sons: London , 2003.

3. F. C. Adams, Elemental Speciation: where do we come from? where do we go?, JAAS, 19 (2004) 1090-1097.

4. A. M. Ure and C. M. Davidson, Chemical Speciation in the Environment, Blackie Academic \& Professional: London, 1995.

5. S. J. Ray, F. Andrade, G. Gamez, D. McClenathan, D. Rogers, G. Schilling, W. Wetzel, G. M. Hieftje, Plasma-source mass spectrometry for speciation analysis: state-of-the-art, J. Chromatography A, 1050 (2005) 3-34.

6. T.O. Berner, M.M. Murphy, R. Slesinski, Determining the safety of chromium tripicolinate for addition to foods as a nutrient supplement. Food and Chemical Toxicology, 42 (2004) 1029-1042.

7. M. Valko, H. Morris, M.T.D. Cronin, Metals, Toxicity, and Oxidative Stress. Current Medicinal Chemistry, 12 (2005) 1161-1208.

8. V. Majidi, M. Moser, C. Lewis, W. Hang, F. L. King, Explicit Chemical Speciation by Microsecond Pulsed Glow Discharge Time-of-Flight Mass Spectrometry: Concurrent Acquisition of Structural, Molecular and Elemental Information, J. Anal. At. Spectrom., 15 (2000) 19-25.

9. L. Li, J. T. Millay, J. P. Turner, F. L. King, Millisecond pulsed radio frequency glow discharge time of flight mass spectrometry: temporal and spatial variations in molecular energetics. Journal of the American Society for Mass Spectrometry 15 (2004) 87-102.

10. R. K. Marcus, Glow Discharge Plasmas as Tunable Sources for Elemental Speciation. In R. C. Cornelis (Ed.), Elemental Speciation: Techniques and Methodology (334-355), John Wiley and Sons: London , 2003.

11. F. Aubriet, C. Poleunis, P. Bertrand, Capabilities of static ToF-SIMS in the differentiation of first-row transition metal oxides, J. Mass Spectrom., 36 (2001) 641-651.

12. C. L. Lewis, M. A. Moser, D. E. Dale, W. Hang, C. Hassell, F. L. King, V. Majidi, Time-gated pulsed glow discharge: Real-time chemical speciation at the elemental, structural, and molecular level for gas chromatography timeof-flight mass spectrometry. Analytical Chemistry 75 (2003) 1983-1996.

13. F. L. King, W. W. Harrison, Glow discharge mass spectrometry: an introduction to the technology and its utility, Mass Spectrom. Rev., 9 (1990) 285-317.

14. F. L. King, C. Pan, Temporal signal profiles of analyte species in modulated glow discharge plasmas, Anal. Chem., 65 (1993) 735-739. 
15. J. Teng, C. M. Barshick, D. C. Duckworth, S. J. Morton, D. H. Smith, F. L. King, Factors influencing the quantitative determination of trace elements in soils by glow discharge mass spectrometry. Appl. Spectrosc., 49 (1995) 1361-1366.

16. F. Aubriet, C. Poleunis, J. F. Muller, P. Bertrand, Laser ablation and secondary ion mass spectrometry of inorganic transition-metal compounds. Part I: comparison between static ToF-SIMS and LA-FTICRMS, J. Mass Spectrom., 41 (2006) 527-542.

17. C. Pan, F. L. King, Atomic emission spectroscopy employing a pulsed radiofrequency powered glow discharge, Appl. Spectrosc., 47 (1993) 2096-2101.

18. C. Pan, F. L. King, Direct Determination of Trace Elements in Graphite Matrices Using Modulated Glow Discharge Atomic Absorption Spectrometry, Appl. Spectrosc., 47 (1993) 300-304.

19. R. E. Steiner, C. L. Lewis, F. L. King, Tim-of-Flight mass spectrometry with a pulsed glow discharge ionization source, Anal. Chem., 69 (1997) 17151721.

20. R. E. Steiner, C. L. Lewis, V. Majidi, Consideration of a millisecond pulsed glow discharge Time-of-Flight mass spectrometry for concurrent elemental and molecular analysis, J. Anal. At. Spectrom., 14 (1999) 1537-1541.

21. C. L. Lewis, G. P. Jackson, S. K. Doorn, V. Majidi, F. L. King, Spectral, spatial, and temporal characteristics of a millisecond pulsed glow discharge: copper analyte emission and ionization, Spectrochim. Acta, 56B (2001) 487-501.

22. L. Li, C. M. Barshick, J. T. Millay, A. V. Welty, F. L. King, Determination of bromine in flame-retardant plastics using pulsed glow discharge mass spectrometry, Anal. Chem., 75 (2003) 3953-3961. 